\title{
Sui fenomeni di anormale abbassamento del suolo, con particolare riguardo al Delta Padano
}

\author{
P. Calloi
}

Ricevuto il 30 Maggio 1967

\begin{abstract}
Riassunto. - Sulla base di ricerche condotte dal 1956 al 1958, nel Maggio 1959 l'A. portava a termine un'ampia relazione sui fenomeni di sprofondamento in atto - a quellepoca - nel Delta Padano. Tale relazione si concludeva con la provata necessità della immediata chiusura delle migliaia di pozzi di estrazione di acque metanifere, ai quali doveva attribuirsi la quasi totalità delle enormi flessioni del terreno, osservate nel Delta. A riprova di tali conclusioni, l'A. riassumeva - in due Appendici - l'andamento di due (fra i tanti) fenomeni di anormale sprofondamento. osservati nel mondo, e chiaramente attribuibili a cause analoghe.

A distanza di nove anni dalla redazione di quei lavori, convinto della validità e della attualità delle conclusioni ivi raggiunte, l'A. ha ritenuto utile pubblicarli, facendoli precedere da una "premessa" e seguire da opportuni raffronti, dai quali balza evidente il monito circa le conseguenze di ventilate - e, in parte, già in via di sviluppo - indiscriminate estrazioni di acque o di idrocarburi in vaste zone dell'alto Adriatico. Ciò che si sta verificando nella zona di Venezia, costituisce già un motivato allarme.
\end{abstract}

SUMmaRY - Basing on researches conducted from 1956 to 1958, the A. completed in Hay 1959 a comprehensive study about the phenomena of subsidence occurring at the time in the region of the Po delta. In its conclusion, the study indicated the prove necessity to close down the thousands of wells for the extraction of methaniferous waters, to which nearly all the enormous subsidences of the ground observed in the Delta were to be imputed. To substantiate his conclusions, the A. resumed -... in two appendices - the course of two (among many) phenomena of $a b$ normal subsidences observed over the globe, and undoubtedly induced by analogous causes.

Now, nine years after their redaction and convinced of the soundness and actuality of the conclusions reached, the A. esteemed appropriate to have these studies published; he introduces them by a " premise " and adds a sequel drawing convenient comparisons, from which issues clearly the warning against the consequences of proposed and, partly, already develoying, indiscriminate extractions of water or natural gases in vast zones of the upper Adriatic. The latest course of events in the Venice region constitutes by itself a motivated alarm already. 


\section{Premessa.}

Per circa un decennio — dal 1956 al 1965 - ebbi ad occuparmi dell'allarmante fenomeno di abbassamento del suolo nel Delta padano e nel Polesine in generale. Tale fenomeno aveva cominciato ad assumere proporzioni preoccupanti a partire dal 1951.

Il mio impegno fu particolarmente assiduo dal 1956 al 1960. Dopo un periodo di indagini orientative, già nel 1957 mi ero convinto - sulla base dei risultati del primo gruppo di ricerche - che la causa predominante della flessione del Delta doveva ricercarsi nella abnorme quantità d'acqua metanifera, che era in corso di estrazione dal sottosuolo polesano. Forte di questa convinzione, dedicai il 1958 alla raccolta degli elementi che portavano a questa conclusione, e alla confutazione degli argomenti che la parte interessata, spalleggiata da alcuni esperti, aveva portato e continuava a ripetere, contro la ventilata chiusura dei pozzi metaniferi.

Nel 1959 terminai la redazione di una prima relazione sullo scottante tema, seguita da due note su analoghi vistosi fenomeni osservati all'estero (in particolare, in California) in analoghe condizioni.

Ritengo utile pubblicare quei tre lavori che - a mio avviso conservano tutta la loro attualità. La graduale chiusura dei pozzi metaniferi del Delta, imposta a partire dal 1960, ha dimostrato quanto ero nel giusto nelle conclusioni di quel mio primo lavoro del 1959 . Il fatto però che l'estrazione di acque metanifere continui in zone marginali del Delta, desta nuove fondate preoccupazioni. Non va dimenticato che, limitrofe alla zona soggetta ad un abbassamento che, in alcuni punti, ha superato i 3 metri e mezzo in circa un decennio, si trovano città come Venezia e Ravenna, in cui - negli ultimi anni il sottosuolo sta subendo flessioni che possiamo definire anormali. Vale la pena di nulla trascurare nella ricerca delle cause, a cui quelle flessioni possono essere attribuite. Il patrimonio culturale, artistico, umano, spirituale - incalcolabile e insostituibile, in quanto, sotto molti aspetti, unico - legato al nome di Venezia (e, sia pure in minore misura, a quello di Ravenna) suggeriscono di sottoporre a rigorose indagini ogni possibile fonte di perturbazione dell'equilibrio dinamico, associato alla salvaguardia di quelle città e di altri luoghi abitati (fra i quali, e fra i più minacciati, Chiogrgia).

Roma, Maggio 1967. 


\section{SULLE CAUSE DELLABBASSAMENTO DEL DELTA PADANO}

(Redatto all inizio del 1959)

1. - Le ricchezze di superficie o del sottosuolo sono per me, come italiano, riccherze della mia terra, e, come tali, preziose. Più che naturale quindi la serenità, e l'interesse, con cui guardo alle une $\theta$ alle altre, spiacente se queste o quelle vengono comunque compromesse. Questo preambolo non avrebbe senso, se l'asprezza delle polemiche, passate ed in atto, sul preoccupante fenomeno, non potesse indurre una delle parti a sospettare che il mio giudizio sia comunque influenzato a favore dell'altra.

Per costume, rifuggo dall'esprimere opinioni, che non nascano da un'intima persuasione. Nel caso specifico, poi, non ho legami di sorta di convenienza o, tanto peggio, di interesse - con nessuna delle due parti: quella che si occupa dell'estrazione del metano e quella dei bonificatori.

Dico questo perchè, in ciò che segue, mi occupo quasi esclusivamente di discutere alcune - non sempre meditate - affermazioni, fatte da funzionari del C.I.M. (Consorzio Italiano Metano), e da loro consulenti. $\mathbf{E}$ ciò per il semplice fatto che nell'anno decorso, ho ricevuto diecine di articoli, di gionnali o di rotocalchi, pubblicazioni di interviste, conferenze-stampa, esami critici, ecc. da una sola delle due parti: quella del C.I.M.

Ciò premesso, entro subito in argomento.

E stato ripetuto, a più riprese, che le inondazioni degli ultimi anni, e particolarmente quella del 1951, sarebbero in gran parte responsabili derli abbassamenti sopravvenuti nel Delta padano. L'ipotesi non meriterebbe di essere presa in considerazione, tanto è assurda. Ad ogni modo, va ugualmente confutata.

Vediamo di calcolare il valore approssimativo della depressione, determinata negli strati superficiali, dalla grande alluvione del 1951.

Riferiamoci ad un sistema di assi triortogonali $x, y$, $z$. Identifichiamo il piano coordinato $x y$ come piano orizzontale e supponiamo l'asse $z$ diretto positivamente verso il basso. Le componenti $u, v, w$ dello spostamento elastico in un punto di un mezzo elastico isotropo, 
causato da una distribuzione superficiale di materia pesante. sono date da Boussinesq nella seguente torma:

$$
\begin{aligned}
& u=-\frac{1}{4 \pi \mu} \frac{\partial^{2}}{\partial x \partial z} \int r d P-\frac{1}{4 \pi(\lambda+\mu)} \frac{\partial}{\partial x} \int \lg (z+r) d P, \\
& v=-\frac{1}{4 \pi \mu} \frac{\partial^{2}}{\partial y \partial z} \int r d P-\frac{1}{4 \pi(\lambda+\mu)} \frac{\partial}{\partial y} \int \lg (z+r) d P, \\
& w=-\frac{1}{4 \pi \mu} \frac{\partial^{2}}{\partial z^{2}} \int r d P+\frac{2 \lambda+3 \mu}{4 \pi \mu(\lambda+\mu)} \int \frac{d P}{r},
\end{aligned}
$$

dove $\lambda, \mu$ sono Ie costanti di Lamè, che caratterizzano il corpo elasticamente, e l'integrazione rispetto alla pressione $P$ nel punto $\left(x^{\prime}, y^{\prime}, 0\right)$ si intende estesa all'area gravata. Il raggio vettore $r$ è dato da:

$$
r^{2}=\left(x-x^{\prime}\right)^{2}+\left(y-y^{\prime}\right)^{2}+z^{2} .
$$

Ora

$$
\begin{aligned}
& \frac{\partial r}{\partial z}-\frac{z}{r}, \\
& \frac{\partial^{2} r}{\partial z^{2}}-\frac{1}{r}-\frac{z^{2}}{r^{3}} .
\end{aligned}
$$

Alla superficie quindi $(z=0), \frac{\partial^{2} r}{\partial z^{2}}=\frac{1}{r} ;$ per cui

$$
\frac{\partial^{2}}{\partial z^{2}} \int r d P=\int \frac{\partial^{2} r}{\partial z^{2}} d P=\int^{2} \frac{d P}{r} .
$$

Nella $3^{a}$ della [1] pertanto

$$
-\frac{1}{4 \pi \mu} \frac{\partial^{2}}{\partial z^{2}} \int r d P-\frac{2 \lambda+3 \mu}{4 \pi \mu(\lambda+\mu)} \int \frac{d P}{r}-\frac{\lambda+2 \mu}{4 \pi \mu(\lambda+\mu)} \int \frac{d P}{r} \text {. }
$$

Lo spostamento verticale sul piano orizzontale diviene allora:

$$
w_{0}=\frac{\lambda+2 \mu}{4 \pi \mu(\lambda+\mu)} \int \frac{d P}{r} .
$$

Analogamente, lo spostamento orizzontale per un'area circolare compressa diviene:

$$
U=-\frac{1}{4 \pi(\lambda+\mu)} \int \frac{1}{r} \frac{\partial r}{\partial a} d D
$$

dove $a^{2}=x^{2}+y^{2}$, il centro del cerchio essendo stato preso come origine delle coordinate. 
A noi interessano qui gli spostamenti verticali, espressi dalla [2].

Date le approssimazioni consentite in quest'ordine di calcoli, possiamo ricorrere ad una semplificazione, quale può essere quella di ritenere circolare l'area sotto pressione. Ciò malgrado, gli sviluppi analitici richiesti, per dare - in questa ipotesi - forma esplicita alla [2] sono alquanto complessi e verranno pertanto omessi.

Per pressione uniforme sopra un'area circolare di raggio $R$, la deformazione verticale in superficie, per un punto esterno all'area considerata, assume l'espressione

$$
w_{o}=\frac{\lambda+2 u}{\pi \mu(\lambda+\mu)} p_{n} a\left\{E(k)-k^{2} \boldsymbol{K}(k)\right\}
$$

dove $p_{o}$ esprime la pressione per area unitaria, $k=\frac{R}{u}, k^{\prime}=11-\frac{\overline{R^{2}}}{a^{2}}$, e $K(k)$ e $E(k)$ sono integrali ellittici completi di prima e seconda specie rispettivamente.

Sulla periferia ̀̀ $\frac{R}{a}=1, k^{\prime}=0, E(k)=E(1)=1\left(^{(1)}\right.$ e quindi la [3] diviene:

$$
w_{o}=\frac{\lambda+2 \mu}{\pi \mu(\lambda+\mu)} p_{o} a
$$

In un punto interno $(R>a)$, si ha

$$
w_{o}=\frac{\lambda+2 \mu}{\pi \mu(\lambda+\mu)} p_{o} R E(\chi)
$$

con $\chi=\frac{a}{R}$. La [3] e la [5] coincidono quindi per $R=a$. Al centro sarà allora (per $a=0$ ):

$$
w_{o}=\frac{\lambda+2 \mu}{\pi \mu(\lambda+\mu)} p_{o} R E(0)
$$

dove $E(0)=1,5708\left(^{2}\right)$.

Per l'applicazione delle formule riportate, mi sono valso dei dati tratti da una pubblicazione dell'Ente Metano: 2 miliardi di metri cubi d'acqua sopra un'estensione di $1000 \mathrm{~km}^{2}$. Cio porta ad uno spessore medio d'acqua di $2 \mathrm{~m}$, su un raggio di $17 \mathrm{~km}$ ca. 
Valgono quindi i seguenti valori numerici:

$$
\begin{aligned}
o_{o} & =1 \\
g & =9,81 \cdot 10^{2} \mathrm{~cm} / \mathrm{sec}^{2} \\
h & =200 \mathrm{~cm} \\
\mu & =4,5 \cdot 10^{10} \mathrm{c} \cdot \mathrm{g} \cdot \mathrm{s} . \\
\sigma & =0,38 \\
\lambda & =\frac{2 \sigma}{1-2 \sigma} \cdot \mu=14,265 \cdot 10^{10} \mathrm{c.g.s.} \\
R & =17 \cdot 10^{5} \mathrm{~cm} .
\end{aligned}
$$

Dalle [4] e [6], per $p_{o}=g \varrho_{0} h$, si ottiene

$$
\begin{array}{ll}
w_{0}=2,9 \mathrm{~cm} & \text { alla periferia, } \\
w_{0}=4,55 \mathrm{~cm} & \text { al centro. }
\end{array}
$$

Tali valori, ripeto, non possono essere che approssimativi: in queste valutazioni, ad ogni modo, è l'ordine di grandezza che conta; e questo è, senz'altro, attendibile.

Al centro dell'area sommersa la flessione del suolo per il carico liquido non deve quindi aver raggiunto i $5 \mathrm{~cm}$.

Qui però va fatta subito un'importante osservazione.

Tale flessione dev'essere stata raggiunta nell'ultima fase dell'allagamento: appena l'acqua ha iniziato a defluire verso il mare, hanno pure principiato la decompressione e il lento ritorno del suolo verso il precedente livello (fatta astrazione dei depositi lasciati - nel frattempo - dalle acque melmose).

Il suolo, infatti, è elastico, come del resto qualsiasi corpo solido, e come è provato dal fatto che trasmette le onde sismiche.

Nel caso specifico, altre prove lo testimoniano, in modo continuo.

Presso l'idrovora di Sadocca (Valle Sabbioni), sulle rive dell'Adriatico, funziona dal Dicembre 1957, una coppia di fotoclinografi. Dal momento in cui è entrata in funzione, la componente EW - e quella soltanto, il mare essendo ail Est della postazione clinografica - registra regolarmente l'alternarsi della marea. Nelle Figg. 1) 2) si riportano alcuni chiari esempi della fedeltà con cui l'ondulazione solida, segue - nella fase e nell'ampiezza - quella liquida, da cui è originata. L'alta marea deprime il fondo marino presso la stazione clinografica, richiamando a sè la componente WE; la bassa marea, alleggerendo il peso sul fondo, fa si che questo si decomprima - come respirando - facendo ruotare la verticale apparente del clinografo 


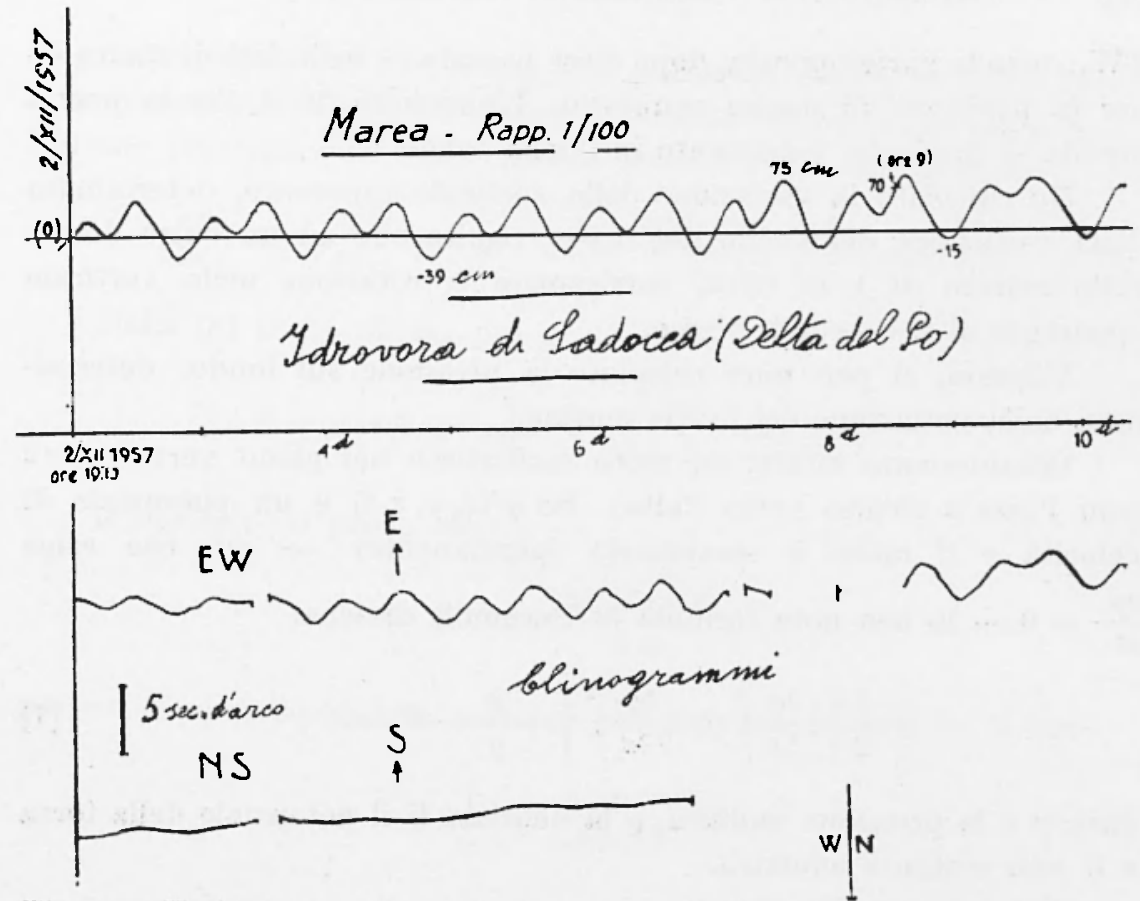

Fig. 1 - Variazione della verticale associata alla marea, registrata dalla comp. EW del clinografo di Sadocca.

marea osseveta $(1 / 100)$

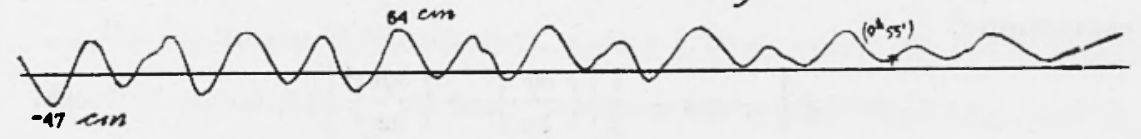

$\frac{\text { Ydrovara di Ladocca }}{\text { (Detta del Po) }}$

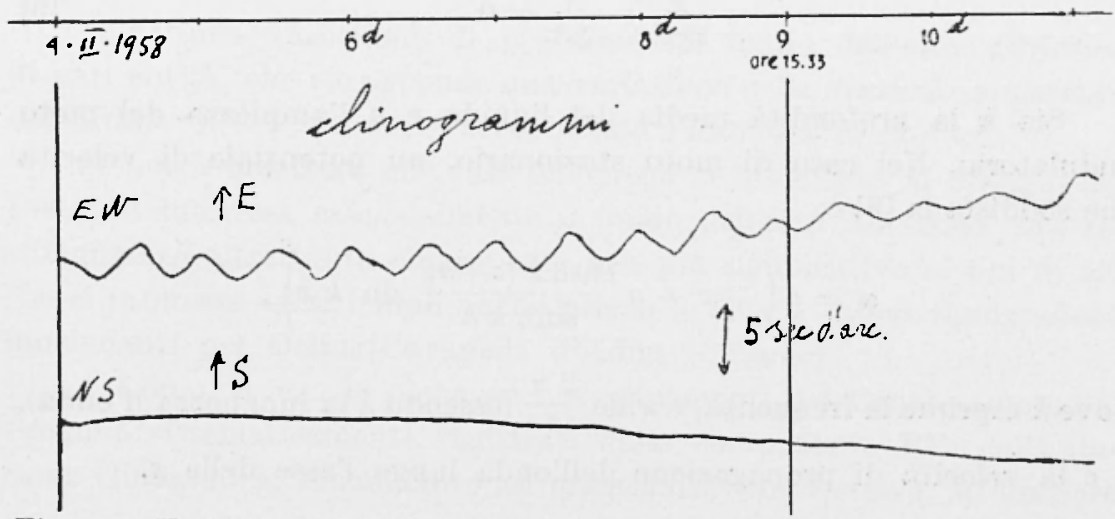

Fig. 2 - Variazione della verticale, associata alla marea, registrata dal clinografo di Sadocea (comp. EW). 
EW, rerso la parte opposta, dopo esser passata - nella fase di stanca per la posizione di medio equilibrio. L'elasticità fa si che la marea liquida si trasformi ferlelmente in quella solida.

Ho calcolato la variazione della verticale apparente, determinata dalla rariazione del livello del mare: risulta che ad un balzo del livello marino di $1 \mathrm{~m}$ circa, corrisponde la rotazione della verticale apparente di 2,5 secondi d'arco.

Volendo, si può pure calcolare la pressione sul fondo, determinata dalla variazione del livello marino.

Consideriamo infatti un moto oscillatorio nel piano verticale $x z$ (con l'asse $z$ rivolto verso l'alto). Se $\varphi(x, y, z, t)$ è un potenziale di velocità e il moto è stazionario (permanente) - ciò che esige $\frac{\partial \varphi}{\partial \tau}=0$ - la ben nota formula di Bernonlli diviene:

$$
\frac{1}{2}\left\{\left(\frac{\partial \varphi}{\partial x}\right)^{2}+\left(\frac{\partial \varphi}{\partial z}\right)^{2}\right\}+\frac{p}{\varrho}-U=C,
$$

dove $p$ è la pressione unitaria, $o$ la densità, $U$ il potenziale della forza e $C$ una costante effettiva.

Nel caso considerato, è:

$$
U=-g z
$$

per cui dalla [7] — prescindendo dalla $C$ - otteniamo per la pressione $p$ l'espressione:

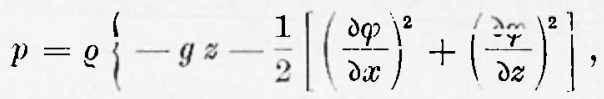

dove $\varphi$ soddisfa alla condizione

$$
\frac{\partial^{2} \varphi}{\partial x^{2}}+\frac{\partial^{2} \varphi}{\partial z^{2}}=0
$$

Sia $h$ la profondità media del liquido e a l'ampiezza del moto ondulatorio. Nel caso di moto stazionario, un potenziale di velocità che soddisfa la [9] è:

$$
\varphi=c\left(-x+a \frac{\cosh k(z+h)}{\sinh k h} \sin k x\right),
$$

dove $k$ esprime la frequenza, e vale $\frac{2 \pi}{\lambda}$ (essendo $i$ la lunghezza d'onda), e $c$ la velocità di propagazione dell'onda lungo l'asse delle $x$. 
Nell'applicazione alle maree dell'Adriatico, dobbiamo tener conto della piccola profondità del mare e delle grandissime lunghezze d'onda. Possono pertanto essere trascurati i termini in $k^{2}$. Ne consegue

$$
\left.\frac{\partial \varphi}{\partial x}\right)^{2}+\left(\frac{\partial \varphi}{\partial z}\right)^{2}=c^{2}\left(1-2 k a \frac{\cosh k(z+h)}{\sinh k h} \cos k x\right) .
$$

Dalla [8] si ha allora, per $z=-h$,

$$
p=\varrho\left[g \ddot{n}-\frac{c^{2}}{2}\left\{1-\frac{2 k a}{\sinh k h} \cos k x\right\}\right] .
$$

D'altronde, si prova che:

$$
c^{2}=g h \frac{\operatorname{tangh} k h}{k h},
$$

per cui - a meno di una costante additiva inessenziale - si ha:

$$
p=\varrho g\left[h+\frac{a}{\cosh k h} \cos k x\right] \text {. }
$$

Pertanto, l'incremento $\Delta p$ di pressione sul fondo, su quello statico, è pari a quello di una colonna d'acqua, corrispondente al termine $\frac{a \cos k x}{\cosh k h}$.

Per la marea dell'Adriatico, dati i piccoli valori di $h$ e i grandi valori di $\lambda . \cosh 2 \pi \frac{h}{\lambda}$ diviene prossimo all'unità. Pertanto, per un valore di a pari a $1 \mathrm{~m}$, si trova per $\Delta p$,

$$
\Delta p=1000 \mathrm{~kg} / \mathrm{m}^{2} \text {. }
$$

E ad una variazione di pressione sul fondo dell'alto Adriatico di pari entità, che corrisponde una variazione della verticale apparente dell'ordine di 2,5 secondi d'arco, presso l'idrovora di Sadocca.

Si potrà obiettare che tale fenomeno si manifesta ai margini del Delta ed interessa essenzialmente il fondo dell'alto Adriatico. Ma testimonianze altrettanto chiare - e ben più significative ai fini di ciò che ci interessa - si hanno anche presso le altre stazioni clinografiche funzionanti nel Delta [Cavanella d'Adige - Loreo].

A Loreo, p. es., le piene ed i straripamenti del Canale omonimo, vengono sistematicamente registrati dalla componente $\mathrm{EW}$ della stazione clinografica, funzionante ad oriente del canale stesso. Mi limiterò 
a citare il caso dell'inondazione, ivi verificatasi ai primi di Novembre 1957: in poco più di un'ora, la componente EW ha accusato uno spostamento verso $W$ di ben 30 secondi d'arco (Fig. 3). Dopo il passaggio dell'onda di piena, con il graduale diminuire dello spessore dell'acqua, il clinometro riprende lentamente a spostarsi in senso contrario, verso la primitiva posizione d'equilibrio, posizione che raggiunge qualche settimana dopo, ad inondazione esaurita.

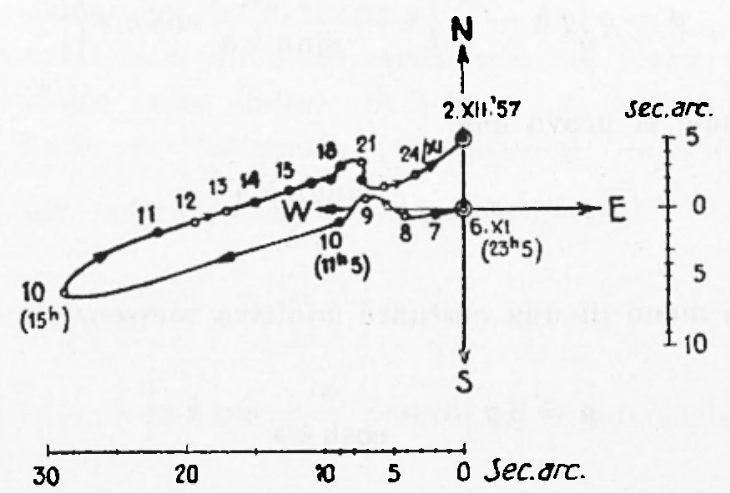

Fig. 3 - Piena, straripamento e corso normale del canale di Loreo, registrati dal clinografo funzionante presso la canonica di Loreo (6-30 Novembre 1957). Graduale crescita livello acqua canale di Loreo 6-10 Novembre 1957. Onda di piena nel canale di Loreo 10 Novembre $1957\left(11^{\mathrm{h}} 5 \div 15^{\mathrm{h}}\right)$. Graduale decrescita livello acqua canale di Loreo 10-30 Novembre 1957.

Dunque, la flessione del Delta determinata dall'alluvione del 1951, una volta rientrate le acque nei fiumi e canali, è andata certamente esaurendosi, riportando sostanzialmente il terreno alle quote precedenti.

Non ha senso quindi imputare a quella alluvione, o ad altre, l'abbassamento del Delta.

A questo punto, è vero, si tirano in ballo dei non meglio precisati fenomeni fisico-chimici, ai quali addossare la colpa di tanto pauroso cedimento.

$\mathrm{Nel}$ caso in esame, di fenomeni fisico-chimici non conosco che quelli che hanno respinto il mare da Torino alle posizioni attuali, negli ultimi millenni; mare che, nel 1300 per esempio, era ancora all'altezza di Donada e di Mesola.

$\mathrm{E}$ tali fenomeni sono appunto intimamente collegati alle alluvioni succedutesi nei secoli; alluvioni che, opponendosi al lento abbassarsi 
della fossa padana, l'hanno via via riempita, portando a valle i prodotti di erosione e distribuendo uniformemente, nel loro libero dilagare, i finissimi depositi di torbida.

Sono gli stessi fenomeni che hanno finito col riempire fosse immensamente più grandi, come quello del Mississippi o del Nilo per non citarne che due. Esistono - è vero - anche i fenomeni contrari, ai quali si deve la formazione dei mari di transgressione; ma rientrano in fatti ben più grandiosi, investendo regioni estese e realizzandosi in una gradualità lentissima.

Quella del 1951 - dal punto di vista naturale - non è stata che una timida ripresa, da parte del vecchio fiume e dei suoi affluenti, dell'antica padronanza di movimenti, che gli aveva consentito di prevalere sul mare, depositando, su un fronte molto ampio, i materiali in sospensione delle sue acque limacciose.

Gli accumuli alluvionali sono essenzialmente conseguenze di estesi, prolungati allagamenti: quando i fiumi non avevano praticamente letto fisso e dilagavano su zone vastissime, or qua or là, depositandovi gli elementi in sospensione, di grossezza decrescente più si allontanavano dai punti di rotta. Le alluvioni degli ultimi anni non sono state quindi che brevi riprese di quelle azioni diluviali - che costituivano la norma in ere geologiche passate - e alle quali si deve il progressivo accumulo di materiali, che ha prevalso sulla flessione del fondo della fossa adrio-padana. L'inondazione del 1951, pertanto, ridistribuendo i materiali di torbida, altrimenti destinati all'Adriatico, sui terreni del Delta ha, caso mai, contrastato il fenomeno di abbassamento, non accelerato.

Ad ogni modo, anche attualmente, è sempre il fiume che prevale sul mare, anche se questo incremento - per dirla con De Marchi $\left(^{3}\right)$ " ̀̀ ora concentrato quasi interamente nell'estremo delta del Po, a che contribuiscono, non tanto le condizioni e le opere locali, quanto le arginature del basso-piano lombardo-veneto che, impedendo le piene trabordanti, conservano fno al mare l'abbondante sfasciume che altrimenti sarebbe distribuito sulla pianura. Ciò che sia, lo prova il confronto col Nilo, il cui delta si accresce assai lentamente, appunto perchè le periodiche piene trabordanti alleviano la massa della maggior parte del carico dejettizion.

A proposito del quale Nilo, se la scappatoia dei fenomeni fisicochimici avesse una parvenza di attendibilità, quel fiume, con le sue periodiche alluvioni, anziché a un delta maestoso, avrebbe dato luogo ad una fossa marina, sul tipo di quella delle Filippine. 
Non is bello, conveniamone, mimetizzarsi dietro simili disinvolte affermazioni.

2. - Quanti, a priori, negano la possibilità che l'estrazione del gas naturale possa comunque contribuire al fenomeno di flessione del delta padano, si sono preoccupati di dimostrare che tale affondamento va senz'altro imputato a cause naturali. Tracciata una carta sulla dinamica del fenomeno, quale si presentava durante il 195i, da essa hanno creduto di poter concludere che l'abbassamento non ha carattere localizzato e discontinuo, ma si estende ad una vasta zona, verso il bordo della quale degrada regolarmente; e che le isocinetiche presentano una caratteristica forma allungata, che si adagia con regolarità, pressappoco sul corso del Po di Venezia. Ciò constatato, veniva spontaneo di afíermare l'origine profonda del fenomeno, il quale doveva comunque essere associato al fiume. L'apporto dei detriti fluviali (15 milioni di tonnellate di materiali solidi all'anno) provocherebbe pertanto la flessione del fondo della fossa.

Non è difficile provare l'insostenibilità di una simile ipotesi.

Il fondo roccioso della fossa padana ha un modulo di rigidità elevatissimo, dell'ordine di $3 \cdot 10^{11}$ poise $\left(\mathrm{g} \mathrm{cm}^{-1} \mathrm{sec}^{-2}\right)$; quello delle alluvioni soprastanti è notevolmente inferiore, non potendo superare (per $v_{s}=500 \mathrm{~m} / \mathrm{sec}$ e $o=2$ ) $5 \cdot 10^{9}$ poise. Ciò significa che la resistenza ai cambiamenti di forma, nel caso del fondo roccioso, è circa 60 volte maggiore che non per il materiale alluvionale: pertanto, la flessione del fondo roccioso, per aumento del soprastante materiale premente, deve risultare enormemente inferiore allo spessore complessivo del materiale alluvionale, che via via si viene aggiungendo. $\mathrm{E}$ che ciò risponda al vero è provato dal fatto che, dalla fine del Terziario, il fondo della fossa padana è andato riempiendosi di materiali d'erosione, così da costringere il mare a ritirarsi, dai margini delle Alpi occidentali alle attuali posizioni. Pertanto, il graduale abbassamento del fondo della fossa padana è stato soverchiato dall'apporto dei materiali d'erosione trasportati dai fiumi. Non si può quindi attribuire a flessione del fondo roccioso l'abbassamento in atto: e se tale flessione viene osservata in superficie, è giusto dire che essa si verifica malgrado l'apporto di materiali, non a causa di esso.

Comunque, l'abbassamento va riportato agli strati superficiali anche per altri motivi, ancora piì incontestabili. Il fondo roccioso non potrebbe flettersi di ben $30 \mathrm{~cm} / a n n o$ (un paio di metri in cinque-sei anni), in zona relativamente ristretta, senza provocare fratture e dislocazioni. 
Atteso l'enorme valore del modulo d'elasticità del fondo (dell'ordine di $\left.6 \cdot 10^{5} \mathrm{~kg} / \mathrm{cm}^{2}\right)$, simili flessioni — in sì breve volger di tempo — sono inammissibili, a meno di violente rotture d'equilibrio. Zone di sismicità elevatissima, come quella intorno a Tolmezzo - dove da un decennio funzionano stazioni sismiche e clinografiche, atte all'osservazione dei lenti movimenti della crosta terrestre - rivelano che lentissime variazioni della verticale apparente, associate a spostamenti superficiali del suolo valutabili in frazioni di millimetro, precedono il verificarsi di scosse sismiche, talora violente: negli ultimi anni, in tal modo i stato possibile sorprendere parecchi terremoti nella loro fase di preparazione.

Cosa ci si dovrebbe aspettare dall'accumulo spaventoso di tensioni che comporterebbe il cedimento del fondo della fossa padana, tanto limitato nello spazio e altrettanto veloce nel tempo? I cataclismi più gravi sarebbero ampiamente giustificati. Per fortuna, nemmeno il più lieve scricchiolio.

No, il fondo della fossa padana non può subire simili flessioni.

Del resto, indipendentemente dalle considerazioni che precedono, le deduzioni tratte dalla carta sulla dinamica del fenomeno, quale si presentava nel 1957, sono state smentite dalle osservazioni successive (Figg. 33-34) L'andamento dei cedimenti non ha conservato aflatto il presunto carattere di omogreneità. Le isocinetiche allora tracciate non costituivano che un episodio della flessione, la quale ha spostato i suoi massimi in altri luoghi, rivelando il carattere accidentale, temporaneo degli abbassamenti, che - nelle loro più cospicue manifestazioni emigrano dall'una all'altra zona del Delta.

Doppiamo concludere che l'abbassamento - almeno nei suoi aspetti più preoccupanti - non può riguardare che gli strati alluvionali: solo in essi agisce la causa delle flessioni più allarmanti.

3. - Passiamo ad un altro argomento, nel quale le Commissioni, nominate dal Consorzio Italiano Netano, ritengono di trovare gli elementi sufficienti per escludere che l'estrazione delle acque metanifere possa essere, anche solo in piccola parte, responsabile della flessione del delta padano. Intendo dire la pressione, quale risulta negli strati alluvionali.

Viene affermato che la pressione idrostatica iniziale, dominante nello strato, è all'incirca uguale a quella normale, in quanto la salienza delle acque è limitata a frazioni di metro. Perciò, per un pozzo alla profondità di 300 metri, con densità media dei materiali sorrastanti 
pari a 2,3 , la pressione di costipamento risulterebbe inizialmente di $10 \mathrm{~kg} / \mathrm{cm}^{2}$ (pari alla pressione di segregazione di $70 \mathrm{~kg} / \mathrm{cm}^{2}$ diminuita della pressione idrostatica, di $30 \mathrm{~kg} / \mathrm{cm}^{2}$ circa). A pozzo in produzione (con pompaggio), si avrebbe una riduzione della pressione idrostatica di $2 \mathrm{~kg} / \mathrm{cm}^{2}$ e quindi un pari aumento della pressione di costipamento, che raggiungerebbe $42 \mathrm{~kg} / \mathrm{cm}^{2}$, con un aumento del $5 \%$ circa.

Sono dati che tolgo da una delle tante relazioni, conferenze-stampa, articoli di giornali o di rotocalco, diffuse dal Consorzio Metano negli ultimi anni.

E da ritenere che, almeno nei primi tempi, il giuoco delle pressioni non fosse quale è stato sopra riportato, se ̀̀ vero che, nei primordi, le acque salate giungevano spontaneamente al limite esterno dei pozzi, senza dover ricorrere - come si fa attualmente - alla tecnica della iniezione di gas nei pozzi stessi.

Ma ammettiamo che ora le cose stiano effettivamente nei termini detti. Non vedo peró come, l'aflermazione categorica del Consorzio Metano che ciò comporti una sola trascurabile diminuzione del volume delle sabbie, sia sostenibile.

L'errore che qui si commette è di considerare l'acqua come elemento dinamicamente separato dal mezzo solido al quale è frammista, quale un semplice riempitivo di spazi che - comunque - resterebbero vuoti o verrebbero ridotti in proporzione minima, qualora essa ne venisse espulsa. Questo può essere sostanzialmente vero nel caso di un mezzo solido, di elevata rigidità, al quale soltanto spettasse il compito di sopportare il peso degli strati soprastanti (sebbene, anche in questo caso, se la pressione andasse oltre un certo limite, risulterebbe inevitabile la riduzione o l'annullamento degli spazi vuoti racchiusi). Ma non è questo il caso in esame. Nel delta padano - specie nella sua parte esterna - noi dobbiamo ritenere la stratificazione alluvionale come un mezzo bifase, investito nella sua totalità della funzione di sostegno.

Un terreno, sabbioso oppure no, costituito oppure no di particelle finissime, sotto la normale pressione presenta interstizi più o meno estesi, piccoli vuoti tra particella e particella, sicché esso potrà assorbire (direi, nascondere) volumi d'acqua più o meno cospicui, senza che questi facciano spazio, senza - in altri termini - che il terreno che la sopporta si gonfi, ampliando il suo volume effettivo.

Però, a mano a mano che tale terreno viene sottoposto a pressioni crescenti, si riducono via via gli interstizi tra particella e particella, e la riduzione - a parità di altre condizioni — sarà tanto maggiore, 
quanto più fini sono gli elementi sciolti del mezzo solido. Al limite, gli interstizi possono totalmente scomparire. Ciò succede effettivamente nel caso di limi sottili, aventi particelle con aree sezionali microscopiche, varianti fra $1,3 \cdot 1,5$ a $1,3 \cdot 2,6$ micron-quadrato (').

Da ciò consegue che, a parità di altre condizioni nei terreni sciolti, più cresce la profondità, più un determinato volume d'acqua diviene spazio effettivo, con funzione portante. $\mathrm{E}$ questo è tanto più rero quanto più il terreno è sciolto, di recente formazione, come appunto la parte esterna del Delta. Come risulta dalle "carote" estratte nelle esperienze degli ultimi anni, ivi il terreno appare, per molti tratti, come un coagulo, una densa emulsione. Sottraete l'elemento liquido a questo mezzo a composizione bifase, sottoposto a pressione, ed avrete contrazione che - in taluni casi - non sarà di molto inferiore al volume del liquido sottratto. 亡̀ chiaro che la consistenza degli strati sovrastanti gioca un ruolo importante nei fenomeni di flessione, conseguente a sottrazione di liquido, flessione che può del tutto mancare nel caso di un'ossatura di rocce, ad elevato modulo di rigidità.

In quasi tutte le interviste, conferenze-stampa, articoli, ece. sopra accennati, si respinge - direi quasi con sdegno - il confronto che taluno osa tentare fra i casi di abbassamenti osservati altrove, e quelli che tanto ci preoccupano. Non mi rendo conto di tanta ostinazione. E evidente che quando, in un modo qualunque, sottraggo nelle profondità del sottosuolo ingenti quantità di materiali, allo stato fluido o allo stato solido, vengo ad alterare le condizioni d'equilibrio negli strati interessati. L'entità e gli effetti dell'alterazione varieranno a seconda dell'ambiente in cui la sottrazione viene eseguita. Per es., in tutto il Brabante, l'estrazione del carbone - intensificata nell'ultimo cinquantennio - ha provocato un aumento della sismicità locale $\left(^{5}\right)$; nel Lodigiano, il soffionissimo esploso presso Basiasco nel 1949, oltre a crepe in una zona circostante, ha causato alcune diecine di terremoti registrati nella stazione sismica di Pavia, alla distanza di $25 \mathrm{~km}$ circa $\left(^{6}\right)$, e gli esempi potrebbero moltiplicarsi. Non vedo quindi perché cause analoghe, sia pure agenti in condizioni diverse, non debbano portary ad analoghi effetti.

Per limitarci al tanto discusso fenomeno di cedimento di Long Beach (California), non capisco perché non debbano essere rilevate le evidenti analogie. Prescindiamo dall'asserzione che le pressioni iniziali di strato ivi sarebbero state enormi - il che, anche fosse stato vero, qui non avrebbe di molto spostato i termini della questione -. Quello che si tiene a mettere in gran risalto sarebbe il fatto che, nella 
zona di Long Beach, l'alfondamento sarebbe strettamente limitato alla superficie del campo di sfruttamento, ciò che non si verificherebbe nel delta padano, dove l'affondamento interesserebbe una superficie di quasi mille chilometri quadrati e decrescerebbe regolarmente dal centro verso i bordi.

Qui, tra parentesi, va rilevata l'insistenza con cui, fino alla vigilia degli ultimi rilievi topografici, ci si sofferma sul presunto carattere di regolarità con cui il fenomeno si manifesterebbe, decrescendo simmetricamente da un centro caratterizzato dal massimo di flessione, in tutte le direzioni. Scopo di questa affermazione - che le ultime misure hanno dimostrato erronea - (v. Fig. 33-34) era di portare una testimonianza a favore del carattere geologico del fenomeno, ritenuto di origine profonda.

Anche sorvolando sul fatto che, in effetti, tutta una vastissima zona del Delta può considerarsi un immenso campo di sfruttamento, la differenza dello spessore degli orizzonti di produzione fra il campo di Long Beach e i campi polesani, verrebbe a spiegare ampiamente la differenza (se esiste) fra gli effetti di flessione osservati nei due casi.

E detto infatti nelle pubblicazioni del Consorzio Metano che, mentre "la potenza degli strati attivi nel campo di Long Beach è di molte centinaia di metri, nei campi polesani gli strati producenti hanno spessori valutabili in diecine di metri ". Se così stanno le cose, si ha pure la spiegazione della presunta differenza fra i casi in questione.

A Long Beach si avrebbe un'alimentazione dei pozzi essenzialmente in senso verticale (cioè locale), laddove nel Delta l'alimentazione non potrì essere che in senso orizzontale, malgrado ciò venga negato dai metanieri. Infatti, viene affermato che le velocità di adduzione delle acque salate verso i pozzi siano dell'ordine dei micron/sec, già a distanze dell'ordine di diecine o, al massimo, di un centinaio di metri. Ciò appare insostenibile: se ciò rispondesse a verità, infatti, un facile calcolo permetterebbe di concludere che la vita media di un pozzo non dovrebbe superare - nel più favorevole dei casi - la durata di un mese, attesa la lieve potenza degli strati attivi. Cosi naturalmente non dev'essere, e così non è.

Ferma restando la potenza degli strati produttivi, l'alimentazione dei pozzi non può che avvenire in senso orizzontale; e, in modo prevalente, da quelle direzioni dove in maggior copia riposano le acque metanifere. Si parla - sempre dalle fonti più volte citate - dell'esistenza di sei vasti bacini pressoché indipendenti gli uni dagli altri, 
in quanto separati da setti impermeabili. E da ritenere - e l'andamento delle cose lo sta a provare - che questi setti presentino vie di penetrazione (inizialmente magari per fenomeni analoghi all'osmosi), che consentano, esaurito un bacino, che i bacini limitrofi comincino a dare il loro apporto a pozzi lontani. Con ciò si spiega il carattere accidentale, temporaneo, degli avrallamenti, e come questi - nelle loro più cospicue manifestazioni - emigrino da una ad altra zona del Delta.

Del resto, indipendentemente da ogni considerazione, il linguaggio delle cifre è davvero eloquente. Nel 1957 — epoca alla quale si riferiscono, in genere, le varie pubblicazioni del C.I.M. - erano in funzione 1500 pozzi. Da ogni pozzo escono giornalmente, in media $500 \mathrm{~m}^{3}$ d'acqua ed altrettanti, per separazione spontanea, di gas metano. Pertanto, una estrazione annua di acqua pari a 275 milioni circa di $\mathrm{m}^{3}$ e altrettanti di gas metano.

Anche quest'ultimo, evidentemente, ha una sua consistenza quando è sciolto nell'acqua salata, così da poter valutare - prudenzialmente - una estrazione annua media di 300 milioni di $\mathrm{m}^{3}$ di acqua metanifera. Una cifra enorme, specie se riferita ai terreni di labilissima compattezza, quali sono quelli interessati dall'emungimento. La flessione massima è - com'è noto - di circa $30 \mathrm{~cm} / a n n o$, in zone relativamente ristrette; in media però possiamo ritenere che nell'area di $800 \mathrm{~km}^{2}$, interessata dall'abbassamento, quest'ultimo non raggiunga i $10 \mathrm{~cm} / a n n o$. Anche in questa ipotesi, avremo una contrazione di terreno dell'ordine di 80 milioni di $\mathrm{m}^{3}$. L'estrazione di $1 \mathrm{~m}^{3}$ d'acqua porterebbe pertanto ad una contrazione, per il terreno che la contiene, minore di $1 / 1$ di $\mathrm{m}^{3}$. Tale cifra, per quanto è stato detto più sopra, è più che attendibile.

Si badi che, giornalmente, ai pozzi corrisponde un'estrazione, di sola acqua salata, pari a circa $750.000 \mathrm{~m}^{3}$. Di contro, la massima flessione giornaliera sarebbe di $8 / 10 \mathrm{di} \mathrm{mm}$ : la flessione varierebbe quindi giornalmente fra zero e $8 / 10 \mathrm{di} \mathrm{mm}$. Cifra enorme, se riferita a fenomeni geologici; ma del tutto giustificabile nei confronti della non meno enorme estrazione di acqua salata.

E qui torna opportuna una preziosa ammissione del Presidente del C.I.M.. Allo scopo di spiegare la possibilità di un abbassamento del Delta da attribuire all'inondazione del 1951 (che, come si è mostrato al n. 1, risulta del tutto infondata; basti osservare che l'inondazione ha portato un sovraccarico di appena $0,2 \mathrm{~kg} / \mathrm{cm}^{2}$ !) egli afferma che "come è noto, le zone deltizie sono sensibilissime a ogni aumento di carico, 
e comunque sono caratterizzate da estrema labilitì di equilibrio dinamico ". Quest'ultima affermazione è senza dubbio vera, e vale sopra tutto nei confronti delle enormi estrazioni di acque salate, di cui si è fatto cenno.

1. - Nelle varie interviste e relazioni, non si manca mai di sottolineare che in altri terreni sedimentari di formazione recente, come nel Delta del Mississippi e in Giappone, dove mai si ̀̀ praticata estra. zione d'idrocarburi, si sono verificati $e$ si verificano abbassamenti imponenti. Nel Delta del Mississippi sarebbero stati riscontrati abbassamenti, mutevoli nel tempo e nello spazio, che raggiungono i $30 \mathrm{~cm} / a n n o$; in Giappone, su zone estese, si sarebbero trovati abbassamenti di 20 cm/anno e oltre. E seguono citazioni di Autori, con titoli di articoli e rlate di pubblicazione.

Confesso che - malgrado il mio scetticismo al riguardo - tali citazioni, fatte con ostentazione di richiami, mi avevano lasciato perplesso. Rispondevano a verità? I fatti mi apparivano troppo fuori dell'ordinario, per non spingermi ad ottenere una conferma.

Ed è appunto ciò che mi sono deciso di fare.

Cominciamo col Giappone.

î nota a tutti l'elevatissima sismicità delle isole giapponesi; sismiciti che si traduce annualmente in un numero enorme di scosse sismiche, molte delle quali di una intensità spesso catastrofica. Tali scosse sono sovente accompagnate da forti sbalzi del terreno; p. es. il disastroso terremoto del Settembre 1923 provocò, nella baia di Aburatubo, un balzo positivo del suolo di un metro e $17 \mathrm{~cm}$, come fu rivelato dal locale mareografo e testimoniato successivamente dalla campagna di Jivellazione, subito iniziata. Nessuna meraviglia, del resto, attese le gigantesche energie che, in simili occasioni, vengono accumulate e, quindi, liberate. Livellazioni di precisione sono frequentissime in Giappone: esse hanno lo scopo di tenere sotto sorveglianza le zone maggiormente soggette ai terremoti. La ripetizione di tali livellazioni è particolarmente curata nelle regioni interessate da faglie attive. Questi rilievi, abbinati alle osservazioni clinografiche ed estensimetriche, hanno rivelato che la grande maggioranza delle scosse sismiche - e la totalità di quelle più intense - sono precedute da lenti movimenti del suolo che - in fase sismica - diventano bruschi e, sovente, di notevole ampiezza. La fase di rottura è sempre preceduta e, sopra tutto, seguita, da miriadi di piccole scosse, che accompagnano le zone in movimento verso nuove posizioni d'equilibrio. 
Ecco perché è da escludere - come è provato al n. 1 - che il fenomeno di anormale abbassamento del delta padano interessi comunque il fondo roccioso della fossa padana.

Cio detto, nessuna meraviglia se, in Giappone, i movimenti della crosta terrestre non costituiscano una novità, associati come sono all'esagerata sismicità di quelle regioni; essi sono mirabilmente riassunti in un lavoro di Miyabe ( $\left.{ }^{7}\right)$, che dà una efficacissima sintesi dei movimenti regionali, in atto in Giappone.

Le mie indagini pertanto non si sono limitate alle zone sismiche. Sfogliando lunghe serie di pubblicazioni giapponesi, su argomenti geofisici e geologici, non mi è accaduto di imbattermi in nessun articolo su abbassamenti del terreno, a carattere anormale, attribuibili a fenomeni geologici.

Mi è accaduto invece - guarda caso - di trovare parecchi studi su fenomeni cospicui di flessione, associati a cause accidentali e, comunque, di superficie.

Mi limiterò a citarne un paio. Un'area di flessione si andava manifestando nel lontano decennio 1920-1930, in una regione del Giappone. Preoccupati dell'entità degli abbassamenti, furono eseguiti dei rilievi di livellazione dal 1925 al 1931.

La depressione, inizialmente lenta, con gli anni era andata accentuandosi; tanto che, nel 1931, alcune case delle località più prossime al mare (Hukagawa), venivano raggiunte dall'alta marea. Una revisione delle livellazioni di precisione, effettuata nella regione nel Luglio 1931, mostrò l'abbussamento subito dai caposaldi in una zona avente una superficie di $30 \mathrm{~km}^{2}$ circa, dal Marzo 1930 al Luglio 1931; abbassamento che nella parte centrale, più depressa, fu di circa $20 \mathrm{~cm}$ (enorme per una zona relativamente ristretta). Ora viene il lato per noi più interessante; nella zona erano attivi, da alcuni anni, tre pozzi di gas metano, proveniente da profondità valutata sui $50 \mathrm{~m}$ circa. E qui riporto testualmente il commento del prof. Miyabe (dell'" Earthquake Research Institute " di Tokyo), esperto di fama mondiale su ricerche del genere, a conclusione del suo primo lavoro sull'argomento:

"It may be interesting to remark that the localities of the wells and the valley in the surface feature of the tertiary beds are fairly coincident with the zone of remarkable depression mentioned above " $\left.{ }^{8}\right)$.

Nel giudizio di Miyabe, quindi, tre soli pozzi producenti metano erano sufficienti a causare flessione in un'area di $30 \mathrm{~km}^{2}$ circa.

Tale giudizio è ribadito nel lavoro successivo $\left({ }^{\circ}\right)$. 
Di eccezionale interesse è pure l'altro caso, che mi appresto a riassumere, in quanto da esso appare evidente la funzione dell'acqua nel mantenimento dell'equilibrio topografico di una regione, specie quando - come nel caso del Delta - i terreni superficiali hanno coefficiente di forma molto basso.

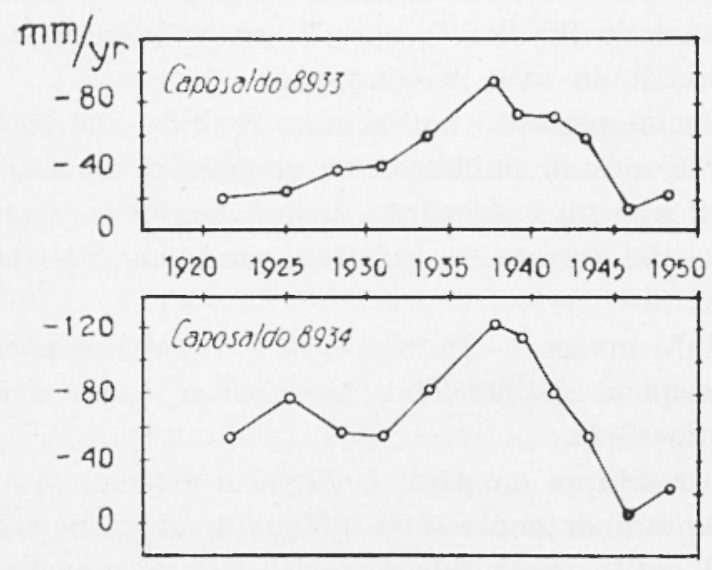

Fig. 4 - Andamento del livello del suolo a Kōto (presso Tokyo) dal 1920 al 1948 (secondo Kishinouye).

Le livellazioni di precisione, compiute dopo il grande terremoto del 1923, rivelarono che nella regione di Kōtō (Tokyo) il terreno eranoin fase di abbassamento. In un primo momento, si attribuì tale fenomeno al terremoto stesso. Senonché, negli anni seguenti, fino al 1938, l'entità del cedimento andò gradualmente aumentando, e si rivelò tanto marcata da divenire oggetto di numerose indagini. Ed ecco il fatto nuovo: durante gli anni fra il 1940 e il 1944-45, l'entità dello spostamento negativo decrebbe rapidamente, tanto che alcuni caposaldi ripresero a risalire (Figg. 4-5). Lo spostamento negativo fu particolarmente piccolo durante l'ultima guerra. Le ricerche condotte da $\mathbf{F}$. Kishinouye, portarono alle seguenti conclusioni. Trascrivo dal testo originale: "The decrease of the rate was considered to be due to the restoring of the pressure of the underground water which might be an effect of the decrease of the amount of water pumped up at factories because of the burning of them by the air-raids. Recently as the factories restored their activities, the underground water-level became lower and lower, and the sinking also became noticeable again " $\left({ }^{10}\right)$. 
A questo riguardo, il livello dell'acqua di un profondo pozzo nel recinto dell'Università di Tokyo (Bunkyō-ku), fu continuamente controllato a partire dal 1932. Il livello diminui di circa $90 \mathrm{~cm}$ dal 1932

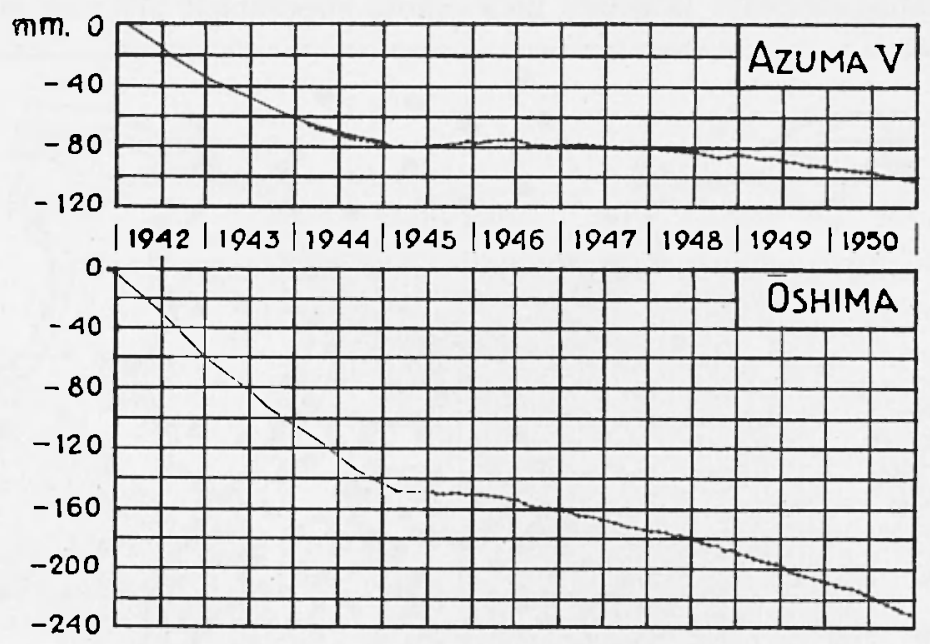

Fig. 5 - Variazioni del livello del suolo ad Azuma ed Ōshima, dal 1940 al 1950. Si noti - come da Fig. 4 - che, interrotto il pompaggio dell'acqua dal sottosuolo, nelle fattorie distrutte per eventi bellici, dal 1944 al 1947, s'interrompe anche il fenomeno di abbassamento (secondo Kishinouye).

al 1943, risali di circa $50 \mathrm{~cm}$ dal 1943 al 1948 e nel 1950 si abbassò di $40 \mathrm{~cm}$ circa sotto il livello del 1948. Così che il livello dell'acqua divenne basso come nel 1943. "Then - continua Kishinouye $\left({ }^{10}\right)$ - the change of the ground height in the Köto region might be closely connected with the water-level. And the condition of the underground water may be an important factor in the sinking of the ground, as was the case in Osaka city " $\left({ }^{11,12}\right)$.

Ma non si arresta qui l'interesse delle ricerche accennate. Esse furono estese a tutta la zona, che circonda la baia di Tokyo, con livellazioni ripetute su parecchie centinaia di caposaldi, fittamente distribuiti su un'area di circa $500 \mathrm{~km}^{2}$ (Figg. 6-7).

Fra l'altro, fu fatto un confronto fra le variazioni degli spostamenti verticali dei caposaldi prima e dopo il 1944. E risultato che in due fascie, ai margini occidentale e settentrionale della zona studiata, vi è un leggero aumento di livello, mentre in tutto il rimanente (in una zona avente una superficie di $400 \mathrm{~km}^{2}$ circa) è in atto un'ampia flessione. Ed ecco il fatto che va fortemente sottolineato. "The sur- 
face geology of the area indicated by $(+)$ is tertiary, and that of the area (-) is alluvial, being perhaps the delta of the Arakawa (viver)".

Dunque, il pompaggio di acqua da un sottosuolo deltizio determina abbassamento sensibile nelle quote superficiali per una zona di

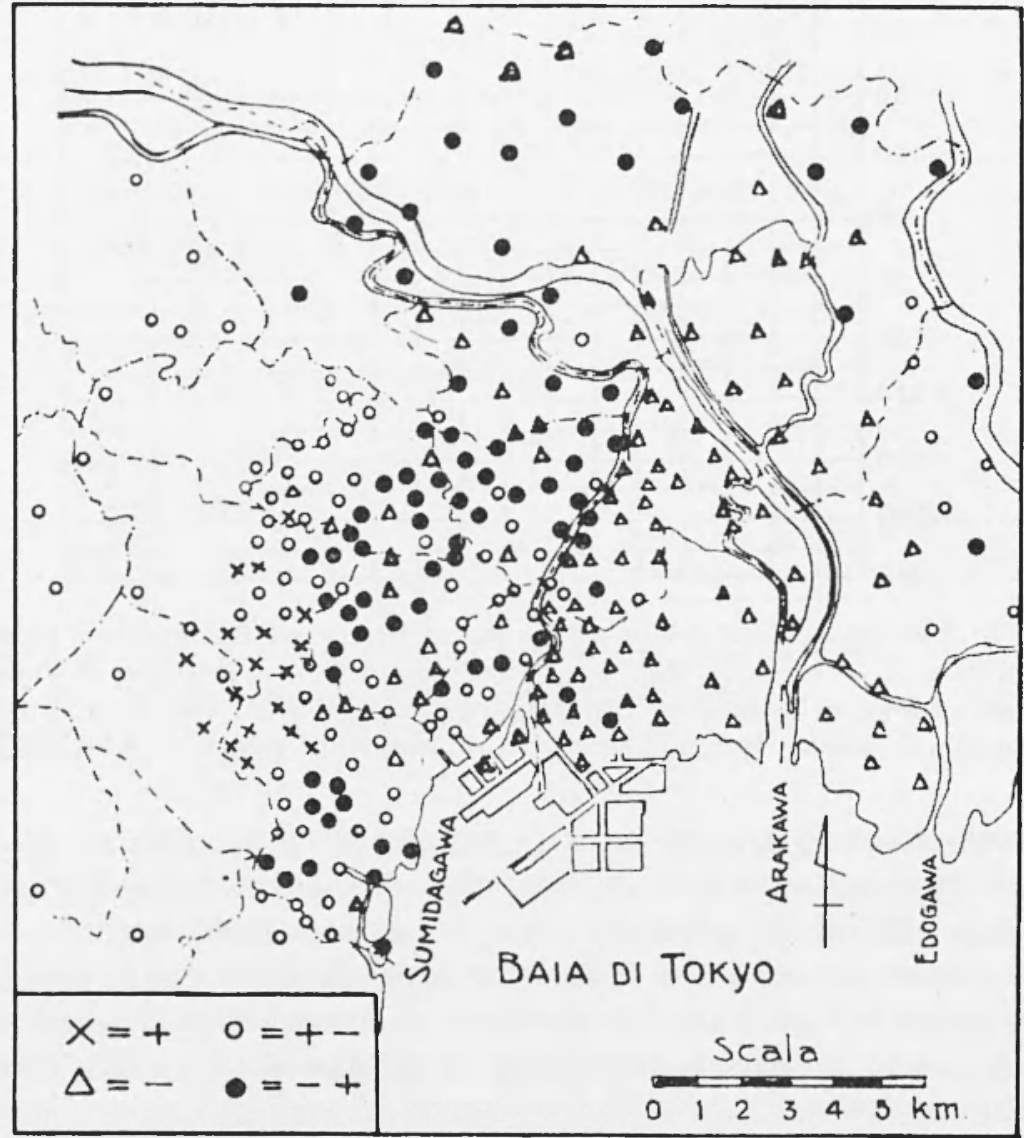

Fig. 6 - Andamento del livello del suolo nella baia di Tokyo (secondo Kishinouye).

notevole estensione. Fatto questo di eccezionale interesse, se si tien conto che la quantità d'acqua ivi estratta è nettamente inferiore a quella che syorga dai 1500 e più pozzi in funzione nel delta padano. Va ancora messo in rilievo che tale fenomeno non interessa terreni più consolidati, quali quelli del terziario.

In zone deltizie recenti, i terreni impoveriti dalle acque si contraggono, coine è provato dalle bonifiche; la flessione però - a 


\section{ERRATA CORRIGE}

La stazione sismica di Serra La Nave sull'Etna di A. Bottari - M. Riuscetti

A pag. 253, riga 5 leggasi:

$$
R=R_{a}+R_{b}+R_{g} \text { in luogo di } R=R_{a}+R_{b}+R_{g}+R_{s}
$$

A pag. 257 Tab. II, colonna 4 leggasi:

"Contenuto medio di silice" in luogo di "Contenuto medio di silicati". 
parità di altre condizioni - è in tali zone ben più accentuata per estrazione di acque profonde, dove l'elemento liquido ha funzione portante.

D'altronde, come dice Kishinouye $\left({ }^{10}\right)$, " the boundary of subsidence of different rate may be that of different thicliness of layer of silt clay

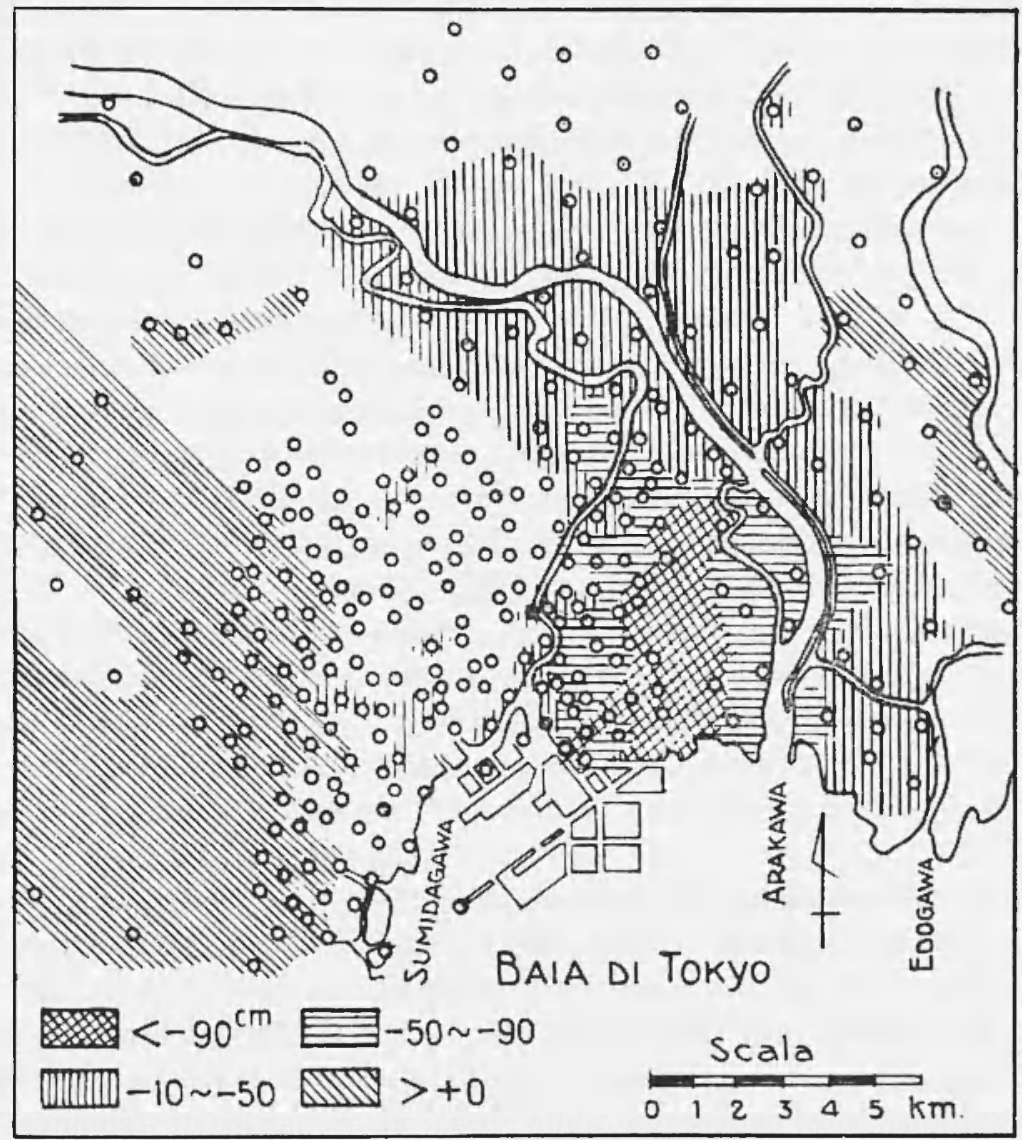

Fig. 7 - Andamento del livello del suolo nella baia di Tokyo (secondo Kishinouye).

(sedimenti argillosi) which contracts through decrease of underground water pressure".

Oltre a interessarmi personalmente dello spoglio di un discreto numero di pubblicazioni giapponesi, ho voluto chiedere l'opinione del Prof. C. Tsuboi, direttore del "Geophysical Institute" dell'Università di Tokyo, il più autorevole esperto sui fenomeni di variazione della crosta terrestre del Giappone. 
In una lettera del 13 Gennaio scorso(*), il Prof. Tsuboi, dopo aver riconosciuto che in alcune regioni del Giappone gli abbassamenti come del resto abbiamo accennato più sopra - sono sensibili, cosi prosegue: "The sinkings have been found by means of repeated precise levels and there can be no doubt about their reality. For instance, in Niigata area, 6 series of precise levels have been made, 1898, 1930, 1941, 1955, 1957, 1958, over a distance of more than $100 \mathrm{~km}$ and $\mathrm{V}-$ shaped sinking (as shown in the diagram) has been found. The largest sinking during 1898-1958 is more than $30 \mathrm{~cm}$.

The rate of sinking has been increasing and the largest rate found

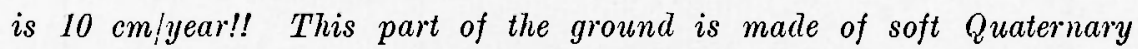
deposit so that some attribute the sinking to the contraction of the deposited material. There can be no doubt that the contraction is one of the causes of this sinking but I personally am a little sceptical if the contraction alone can explain the whole phenomena".

Dunque, in un sessantennio, lungo un profilo di oltre $100 \mathrm{~km}$, il massimo spostamento, verso la parte centrale, di $30 \mathrm{~cm}$ complessivi è ritenuto da Tsuboi molto grande, e portato ad esempio. Inoltre, l'osservazione di un abbassamento recente, in materiale del Quaternario, di $10 \mathrm{~cm} /$ anno porta Tsuboi a trascrivere la notizia, facendola seguire da due punti esclamativi. Quale sarebbe la meraviglia del prof. Tsuboi sui $30 \mathrm{~cm} /$ anno del delta padano, in zona ben più ristretta? E i dubbi espressi alla fine della sua lettera resterebbero ancora tali?

5. - E passiamo all'America e ai famosi abbassamenti di 30 cm/anno, che sarebbero stati osservati nel delta del Mississippi, in zone dove non si estraggono idrocarburi.

Già sapevo dell'esistenza di numerosi e, talvolta, enormi fenomeni di abbassamento, in relazione con campi petroliferi o di cultura metanifera. Non conoscevo nulla peró al riguardo di fenomeni di abbassamento di natura geologica, di comparabile entità.

Mi rivolsi dapprima al massimo Ente statale, incaricato dei livellamenti di precisione negli U.S.A.: l'“U.S. Coast and Geodetic Survey " di Washington. In risposta alla mia lettera, in cui chiedevo conferma, dell'esistenza o meno, di abbassamenti di quella entità, la Direzione del "Coast and Geodetic Survey" mi notificó, fra l'altro, quanto qui trascrivo. "We have no indication of settlement of the magnitude suggested in your letter; however, I am enclosing a copy of the

(*) Si ora nel 1959 . 
results of releveling in the vicinity of New Orleans, Louisiana, which shows a settlement of about thirty centimeters for some bench marks over a period of several years.

We do not have sufficient releveling south of New Orleans in the Delta region to show what the settlement might be there. Tidal observations indicate that, in general, mean sea level is rising at a rate of about 0,021 foot per year in the Gulf of Mexico. This change is probably a combination of a rise in sea level and subsidencen.

Nei documenti allegati, figurano 21 pagine dedicate ai risultati di livellazioni di precisione, compiute nel 1938, 1951 e 1955 su circa 300 caposaldi, in una zona nei pressi di New Orleans; e 53 pagine, dedicate alla descrizione dei caposaldi stessi.

Da un esame di detti risultati si deduce che su centinaia di caposaldi livellati, soltanto in 13 di essi gli spostamenti negativi raggiunsero o superarono di poco i $30 \mathrm{~cm}$; però in un intervallo di $13 \mathrm{anni}$, e pertanto con una variazione annua dell'ordine di due o tre $\mathrm{cm}$. In tutti gli altri caposaldi si ebbero cedimenti molto più piccoli, e in parecchi casi, trascurabili. Nei quattro anni dal 1951 al 1955, i massimi spostamenti negativi si ebbero in tre caposaldi, con abbassamenti dell'ordine di $2 \mathrm{~cm} / \mathrm{anno}$; in una diecina variarono fra 1 e $1,5 \mathrm{~cm} / \mathrm{anno}$; in tutti gli altri (cioè nella grande maggioranza) o furono di pochi millimetri l'anno, oppure nulli, o addirittura positivi (in un caso persino di $+1,1 \mathrm{~cm} / \mathrm{anno})$.

I rilievi interessano un'area di circa $1200 \mathrm{~km}^{2}$.

In detta area, pertanto, nulla che possa lontanamente avvicinare le enormi cifre di abbassamento, riportate nelle pubblicazioni del C.I.M.

Un'altra istituzione statunitense, che non avrebbe potuto ignorare abbassamenti tanto eccezionali, era l'"U.S. Geological Survey", di Washington. Le mie richieste a tale istituzione, ebbero risposte di un'estrema cortesia, accompagnate da mappe e pubblicazioni di notevole valore.

Nella lunga risposta alla mia prima lettera, è detto, fra l'altro:

"The figure you have heard, of 30 centimeters per year for the sinking of the Mississippi delta, seems large to us also. I have checked many sources of information, and the only figures which approach it in magnitude have proved to be misprints".

E più oltre: "As you may know, our Mississippi delta is a complex of individual deltas built at different times as the course of the lower part of the river has shifted its position - seven deltas have been differentiated to date. The maximum reported rate of sinking takes place 
on the present - day delta. This is also the locus of maximum growth of the land area as it encroaches on the Gulf of Mexico. The Mississippi River brings about 500 million tons of sediments to the Gulf each year. One of its distributaries, Southwest Pass, has advanced into the Gulf at about 250 feet per year since $1838 \%$.

E ancora: "Several geologic proccsses contribute to the sinking of the land surface. The delta is located in our most active geosyncline, which parallels the northern coast of the Gulf of Mexico. A thickness of some 9.000 meters of Tertiary sediments reflecting shallow-water deposition, indicates subsidence of about 150 meters over a period of a million years. The mass of later Quaternary delta sediments has caused a structural depression of the underlying deposits. In addition, compaction of the sediments is responsible for a substantial part of the lowering of the surface.

Other geologic events of more local occurence have superimposed movements, either downward or upward, on the regional picture. These include faults (not easily recognized in this type of sediment), salt domes, and mud lumps (accumuli di limo). Mud lumps, described only from the outer part of the present Mississippi delta, are deformed masses of clay which have forced their way up through overlying silty beds ".

Questo un estratto della prima lunga lettera della geologa in carica, Miss Alice Allen.

Poco dopo ricevetti quattro mappe topografiche (scala $1: 62.500$ ), rappresentanti l'area del delta del Mississippi. Ma di particolare interesse mi riuscì l'opera - davvero di eccezionale valore - "Geology of the Mississippi River Deltaic Plain, Southeastern Louisiana " pubblicata, in due volumi, nel Luglio 1958 (e quindi recentissima) dal U.S. Army Engineer Waterways Experimental Station in Vicksburg, Mississippi. Nel primo volume sono riassunte le ricerche, lontane e recenti, condotte nel delta del Mississippi, con dovizia di mezzi: la Valle del Mississippi nell'era glaciale, storia fisiografica e geologica, depositi pleistocenici e loro caratteristiche fisiche, depositi recenti e loro caratteristiche fisiche, ecc. ecc. Il secondo volume (di non comuni dimensioni: $45 \times 65 \mathrm{~cm}$ ), consiste di 27 tavole, nelle quali il Delta viene riportato nei suoi aspetti più disparati con rilievi di superficie e di profondità: geologici, geofisici, chimici, fisici, prospezione superficiale e profonda, determinazioni di date con radio carbonio - 14, ecc. Nel complesso, un'opera fondamentale per la conoscenza del delta del Mississippi, condotta con metodi da prendere ad esempio per indagini del genere. 
Nel volume primo, un ampio capitolo è dedicato alla flessione del Delta, in cui si discutono le varie cause dell'abbassamento, subito dagli strati alluvionali e dalla base rocciosa mesozoica, attraverso le varie epoce. Una delle principali cause di abbassamento è attribuita alla disidratazione degli strati alluvionali (pag. 96), particolarmente accentuata nel Pleistocene, a causa dell'abbassamento del livello del mare; disidratazione che accelera il consolidamento dei sedimenti. Alle varie cause di abbassamento, si somma il progressivo aumento del livello dei mari, determinato dallo scioglimento accentuato dei ghiacciai. Presentemente, l'aumento del livello marino è stimato in $10 \mathrm{~cm}$ per secolo (pag. 96).

In una tavola (pag. 97) sono raccolti gli abbassamenti osservati nei vari luoghi, e nelle diverse epoche, nel Delta, e causati da diversi processi geologici. La massima flessione osservata fu nella "East Bay" nel basso delta, nel periodo 1869-1917, con un cedimento annuo di 0,16 piedi, pari a $\mathrm{cm}$ 4,8. In media, si calcola che l'abbassamento del delta attuale sia di circa 5 o più piedi per secolo, cioè $d i$ circa $\mathrm{cm}$ 1,5 per anno (*). L'entità dell'abbassamento diminuisce nel retroterra.

Ad ogni modo, nessuna traccia dei famosi $30 \mathrm{~cm} / a n n o$, riportati in tante interviste del C.I.M.; e le sorgenti delle notizie qui riportate non potrebbero essere più autorevoli, come autorevolissima appare la preziosa pubblicazione ufficiale del Luglio 1958, di cui qui si è fatto cenno. $\mathrm{Va}$ ancora sottolineato che - come è detto nella prima lettera del "Geological Survey " di Washington - il delta del Mississippi viene a trovarsi nella più attiva geosinclinale degli Stati Uniti, come a dire che, per cause strettamente geologiche, altrove non si verificano, e non sono da aspettarsi, abbassamenti di maggior entità di quelli che si osservano nel delta del Mississippi.

$\left(^{*}\right)$ L'errore di stampa a cui si accenna nella prima lettera, è quello di 2 "feet" per anno (anziché 2 "inches"), ripreso da un lavoro di Russel del $1936\left(^{(3)}\right)$. D'altronde, a parte l'errore $(60 \mathrm{~cm}$ in luogo di 5 !), tale flessione si riferiva allo sprofondamento subito da una cabina telefonica dal 1853 al 1877 , sprofondamento evidentemente legato alla sua particolare forma e al suo peso.

Cadono quindi tutte le illazioni - e speculazioni -, su questa cifra, a più riprese ripetute da funzionari dell'E.M. e da suoi esperti, non ultima quella ribadita negli interventi su una relazione del Prof. Dal Piaz( ${ }^{15}$ ) (pag. 472). 
Un altro particolare mi preoccupavo di chiarire. Si andava affermando che i casi di abbassamento, legati all'estrazione del petrolio o dei gas naturali, fossero pochi in America, e di lieve estensione.

Da quanto ho potuto sapere, risultano invece comunissimi. Naturalmente, ci si limita a discutere di quelli più vistosi e, sopratutto, di quelli che compromettono la sicurezza o la stabilità di luoghi abitati.

A questo proposito, Charles $\mathrm{R}$. Iolb, geologo capo direttore della "Waterways experiment Station" di Vicksburg (Mississippi), rispondendo ad una mia lettera, cosi si esprime: "If, on the other hand, you refer to subsidence connected with withdrawal of natural oil or gas from deeper and geologically much older horizons, there is a substantial amount of literature of such occurrences in the States. Subsidence due to grounlwater withdrawal is also quite common".

Di altri casi di flessione, legati all'estrazione di acque sotterranee, mi parla Miss Allen dell' "U.S. Geological Survey" in una seconrla, lunga lettera del 20 Marzo 1959 (p. es. i problemi dell'abbassamento in atto nella "San Joaquin Valley" in California, attribuito, in gran parte, all'estensiva estrazione di acqua dal sottosuolo). Nella stessa lettera si soflerma sull'ormai famoso abbassamento di Long Beach.

I funzionari del C.I.M., e le commissioni da esso nominate, affermano recisamente non avere tale abbassamento nessuna analogia con quello del delta padano. Abbiamo già osservato al n. 3 quanto sia incauta questa affermazione. In linea generale, dovunque si verificano estrazioni di idrocarburi, in enormi quantitì, sono inevitabili sensibili variazioni nell'equilibrio dinamico delle zone interessate; cose queste che regolarmente si verificano, specie nelle regioni con ossatura geologica poco consistente. Nel caso specifico, si giuoca molto sulle differenze delle pressioni iniziali; ma tali differenze - che qui del resto sono soltanto supposte - non sono determinanti, ai fini della stabilità del sottosuolo. Sono i milioni di metri cubi di sostanze estratte che costituiscono la causa predominante delle grandi flessioni.

Tornando a Long Beach, si suole affermare dalla parte interessata che la zona dell'affondamento è limitata ad una superficie di qualche chilometro quadrato. Ed anche questo non risponde al vero, chè la flessione, massima nella zona di Long Beach, arriva ad interessare anche Los Angeles, a circa quaranta chilometri di distanza. Altro che qualche chilometro quadrato! i vero comunque, che come si ̀̀ detto nella lettera citata — "the surface subsidence overlies the most actively producing oil field in California ... the areal extent 
and the time of the subsidence are closely related to oil production ". Esiste una esauriente pubblicazione su questo caso non comune $\left({ }^{15}\right)$. Nella lettera già citata, si aggiunge che l'abbassamento non ha cessato di continuare: "Subsidence has continued since that date, and was approaching twenty five feet the last I heard. The sea has been liept out by dilies, and the depressed area is being filled in. A tremendous projet is now under way to reduce future subsidence by pumping sea water into the oil producing sands to restore their original pressure. The oil field is made up of six individual fault blocks (with some faulted subdivisions), and there are seven different zones from which oil is produced. The present plan calls for injecting 1.7 billion barrels of salt water over a period of five years. The water will be pumped into the four upper producing zones in five fault blocks, and into all producing zones in another fault block. The objective is to slow down and eventually stop additional subsidence. It is not expected that past subsidence can be corrected".

In tal modo, torna ad essere ancora una volta sottolineata la essenziale funzione portante che ha l'acqua in tutte le zone alluvionali o comunque includenti stratificazioni sabbiose fortemente impregnate. Il progetto è definito tremendo; ciò non ostante non si esita di mandarlo a compimento, pur di immettere nel sottosuolo, quell'elemento liquido (l'acqua), col compito di colmare, almeno in parte, l'enorme depressione, conseguente all'estrazione del petrolio.

6. - Da quanto è stato qui brevemente detto, un fatto balza incontrovertibile: $i$ fenomeni di innalzamento o abbassamento funora osservati nel mondo, quando sono determinati da sole cause geologicheo, comunque, naturali -, sono sempre lentissimi; solo quando ad essi, si sovrappongono cause accidentali, sotto forma di disordinate estrazioni di liquido o di gas in quantità enormi, gli spostamenti negativi vengono dovunque accelerati, specie nelle zone caratterizzate da lieve modulo di rigidità.

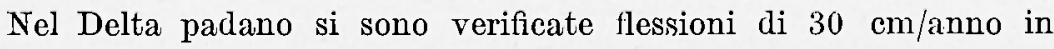
alcune località, nelle zone di estrazione delle acque metanifere; in alcuni luoghi, dal 1951 l'abbassamento complessivo è stato dell'ordine di due metri in pochi anni. Queste cifre non trovano riscontro in fenomeni esclusivamente naturali. Non è ammissibile pertanto insistere con l'affermare - come fa una delle parti interessate - che non esiste nessuna correlazione fra l'affondamento e l'estrazione del gas naturale. 
Come è illusorio credere di risolvere l'assillante problema con le ricerche gravimetriche, con le ricostruzioni geologiche degli orizzonti del Quaternario, con il rilievo topografico del fondo marino, con il prelievo di carote, con lo studio stratigrafico e micropaleontologico, con la sospensione dell'estrazione in uno o in un gruppo di pozzi, ecc. Tali ricerche potranno riuscire utilissime ai fini scientifici, ma, a mio parere, per sanare, se ancora si sarà in tempo, la situazione nel Delta, si deve avere il coraggio di ricorrere ad una soluzione drastica, senza tentennamenti o compromessi. E quale sia questa soluzione, essa viene chiaramente suggerita dal contesto di quanto sono venuto esponendo.

Roma, Maggio 1959. 


\section{COMPLEMENTI AL PRECEDENTE LAVORO SUI FENOMENI DI ABBASSAMENTO DEL DELTA PADANO}

A complemento della precedente relazione, ritengo utile riassumere brevemente le caratteristiche di due notevoli casi di abbassamento del terreno, in atto negli Stati Uniti. Uno (Appendice I) si riferisce alla valle di San Joaquin (California), l'altro (Appendice II) alla zona di Long Beach (California). La zona soggetta ad abbassamento nella San Joaquin Valley ha una superficie nettamente superiore a quella soggetta ad analogo fenomeno del delta padano, sebbene presenti con quest'ultimo strette analogie. Ne tratta diffusamente una monografia della Commissione di studio americana, presieduta da Poland.

L'abbassamento in atto nella zona di Wilmington denuncia eccezionali proporzioni, superando - nella parte più depressa - il valore di $\mathrm{m} \tau, \overline{\mathbf{5}}$. Presentano particolare interesse, in questo secondo caso, i lavori in corso intesi a rallentare e, possibilmente, arrestare la flessione del terreno, attribuito alle notevoli estrazioni di petrolio e di gas; lavori che si esplicano nella immissione, nella zona depressa, di enormi quantità d'acqua marina, sotto opportune pressioni. Dati recenti, relativi a quest'opera, sono tratti da fascicoli di "The Oil and Gas Journal ».

Roma, Gennaio 1960. 


\section{APPENDICE I}

\section{Sullabbassamento anormale della valle di San Joaquin (Ca- LIFORNIA).}

Nella valle di San Joaquin (California), Fig. 8, negli ultimi decenni, è in corso un fenomeno di abbassamento del terreno, che si ̀̀ andato accentuando durante gli ultimi anni $\left({ }^{16}\right)$ : Figg. 9, 10, 11.

G'li studi accurati, condotti con adeguati mezzi dall'"Inter-Agency Committee on Land Subsidence in San Joaquin Valley " hanno portato a riconoscere due tipi di abbassamento: 1) abbassamento di superficie ("shallow subsidence »), limitato a zone poco estese; 2) abbassamento di profondità, di notevole estensione.

1) Abbassamento di superficie. - Il primo tipo di abbassamento (Figs. 11, 11a, 12, 12a, 12b) ha raggiunto - in un posto d'osservazione $-9,5$ piedi di flessione durante $i$ primi 16 mesi di indagine (pag. 86).

I depositi alluvionali che si comprimono apprezzabilmente su inzuppamento, determinando fenomeni di "shallow-subsidence " appaiono essere localizzati a ridosso degli aridi margini occidentale e meridionale della valle, dove la media caduta annua di pioggia è insufficiente a penetrare sotto la zona delle radici ( $"$ the root zone $)$ ). Rade inondazioni torrenziali rappresentano il principale scarico dei rii, che producono i ventilati depositi comprimibili.

Depositi suscettibili di abbassamento di superficie provengono essenzialmente da pendii di scolo brevi, ripidi, frontali, che hanno aree imbrifere relativamente piccole. Le rare, rapide tempeste, insieme alle forti pendenze e alla rada vegetazione di copertura, facilitano la rapida evasione delle acque e la bassa umidità.

Apparentemente, i depositi di limo sono aree caratteristiche di "shallow subsidence ". Depositi sciolti, a bassa densità, aventi alta porosità, sono tipici di queste aree in abbassamento. Depositi eolici in alcune aree presentano tale tendenza.

Fenomeni conosciuti di "shallow subsidence" ad ovest delle contee di Fresno, Kings e Kern sono circoscritti ad una ristretta cintura 


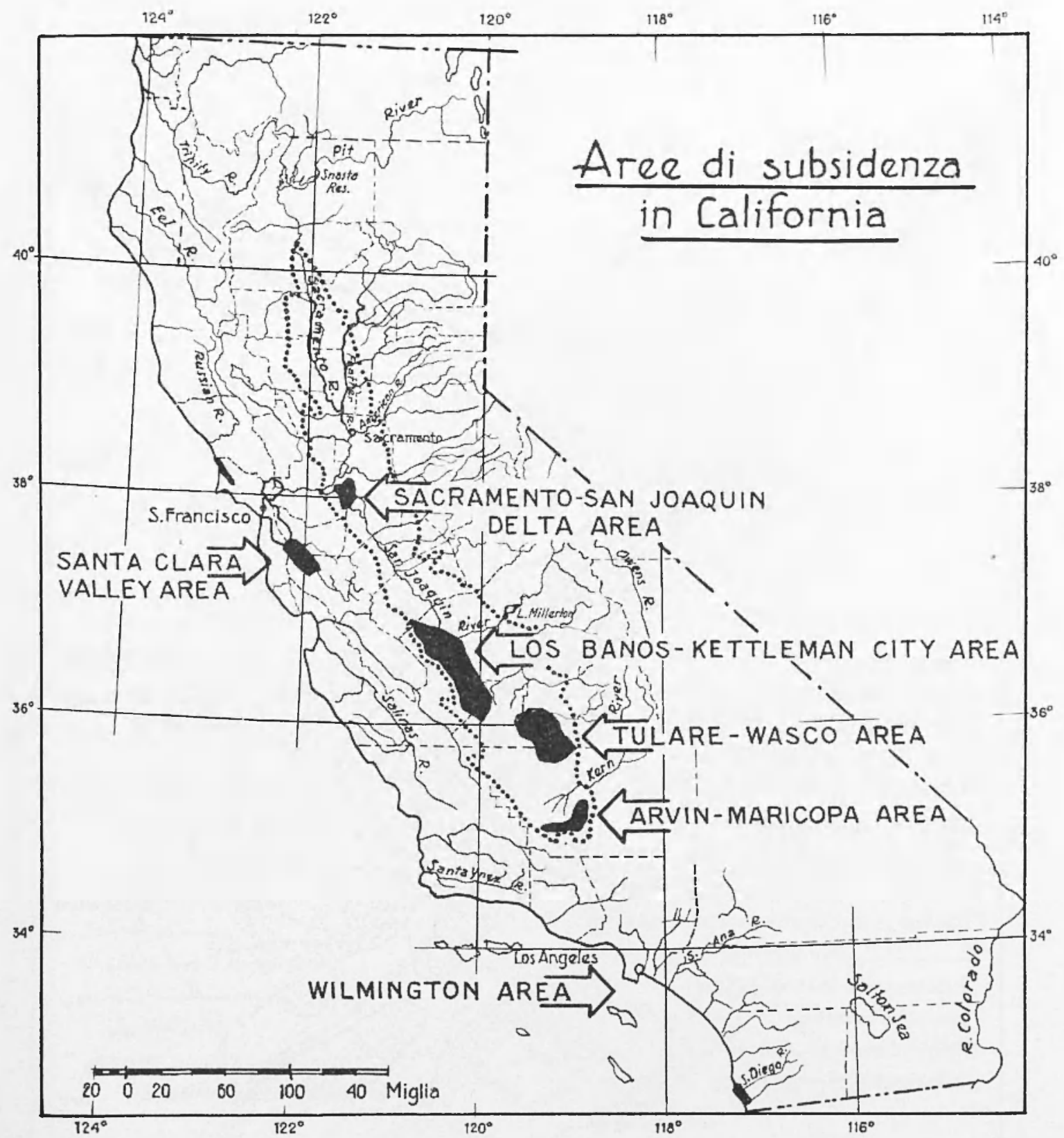

Fig. 8 - Principali aree di abbassamento in California (secondo la "InterAgency Committee on Land Subsidence in San Joaquin Valley - I.A.C."). 


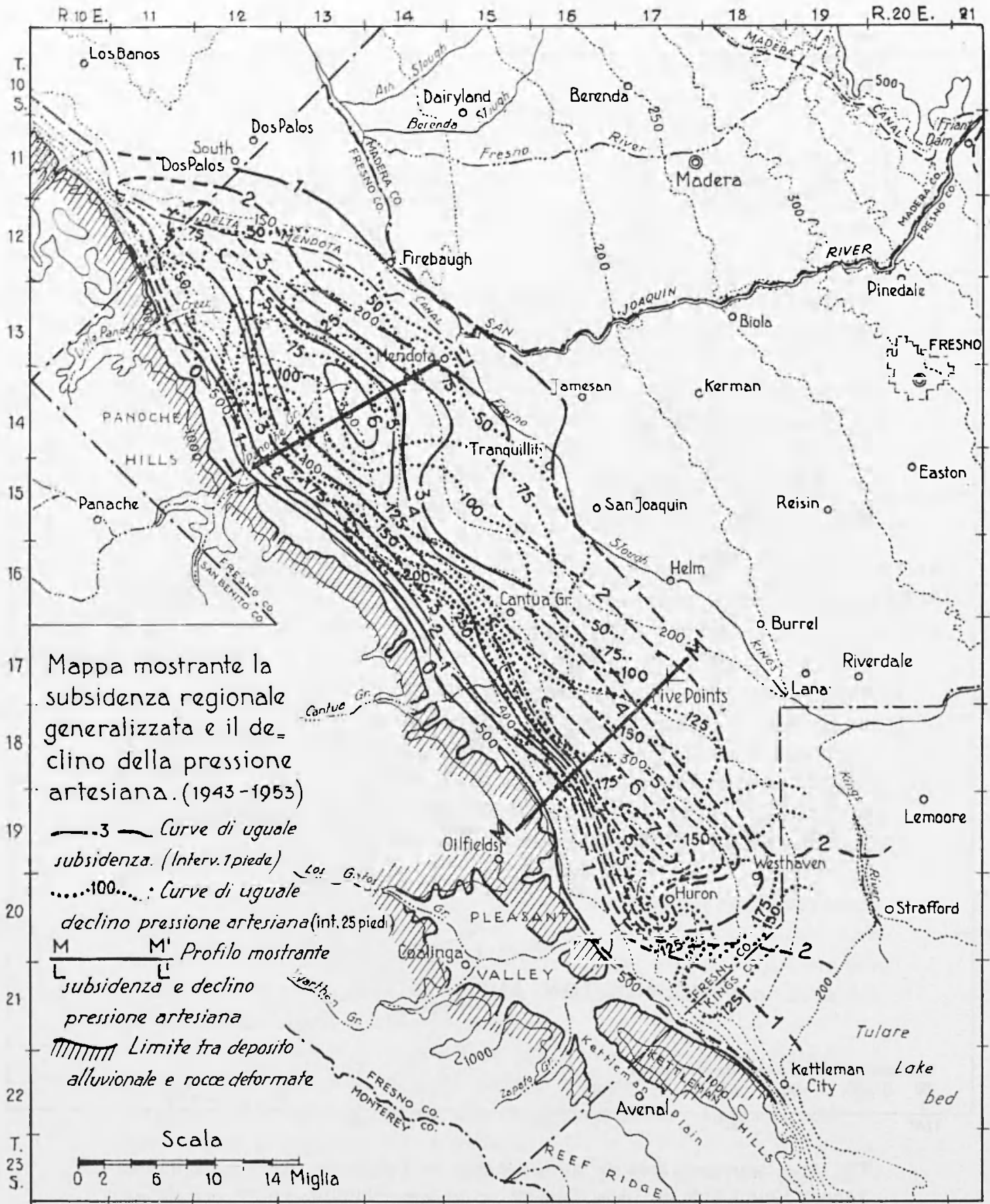

Fig. 9 - Aspetti degli anormali abbassamenti nella San Joaquin Valley (secondo l'I.A.C.). 


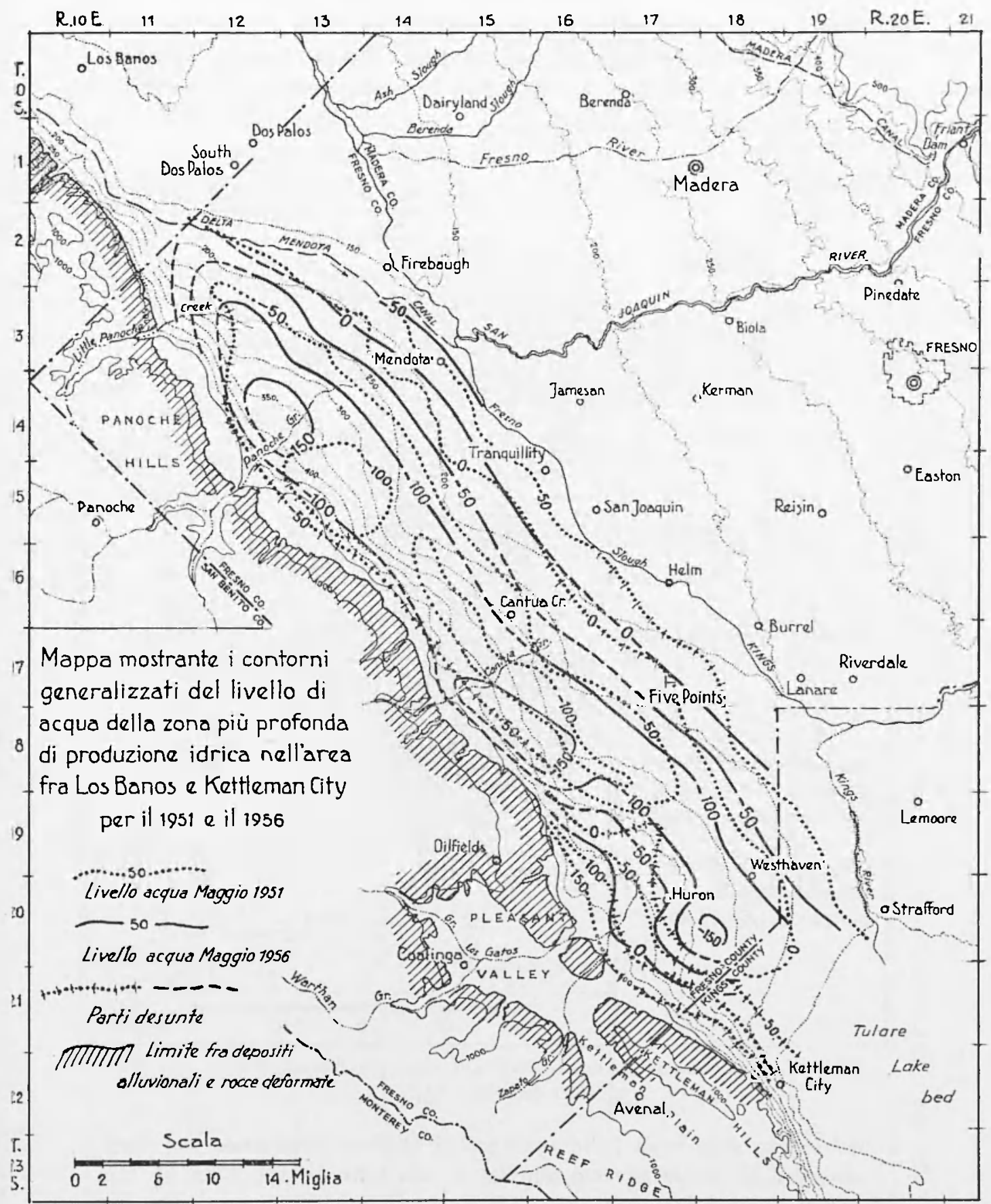

Fig. 10 - Andamento del livello delle acque profonde nella San Joaquin Valley dal 1951 al 1956 (secondo l'I.A.C.). 
di recenti terreni alluvionali, provenienti ad est della "Coast Ranges" e a nord delle Montagne di San Emigdio. Nessun segno di "shallow subsidence" ¿̀ stato osservato in vecchi terreni alluvionali o in terreni

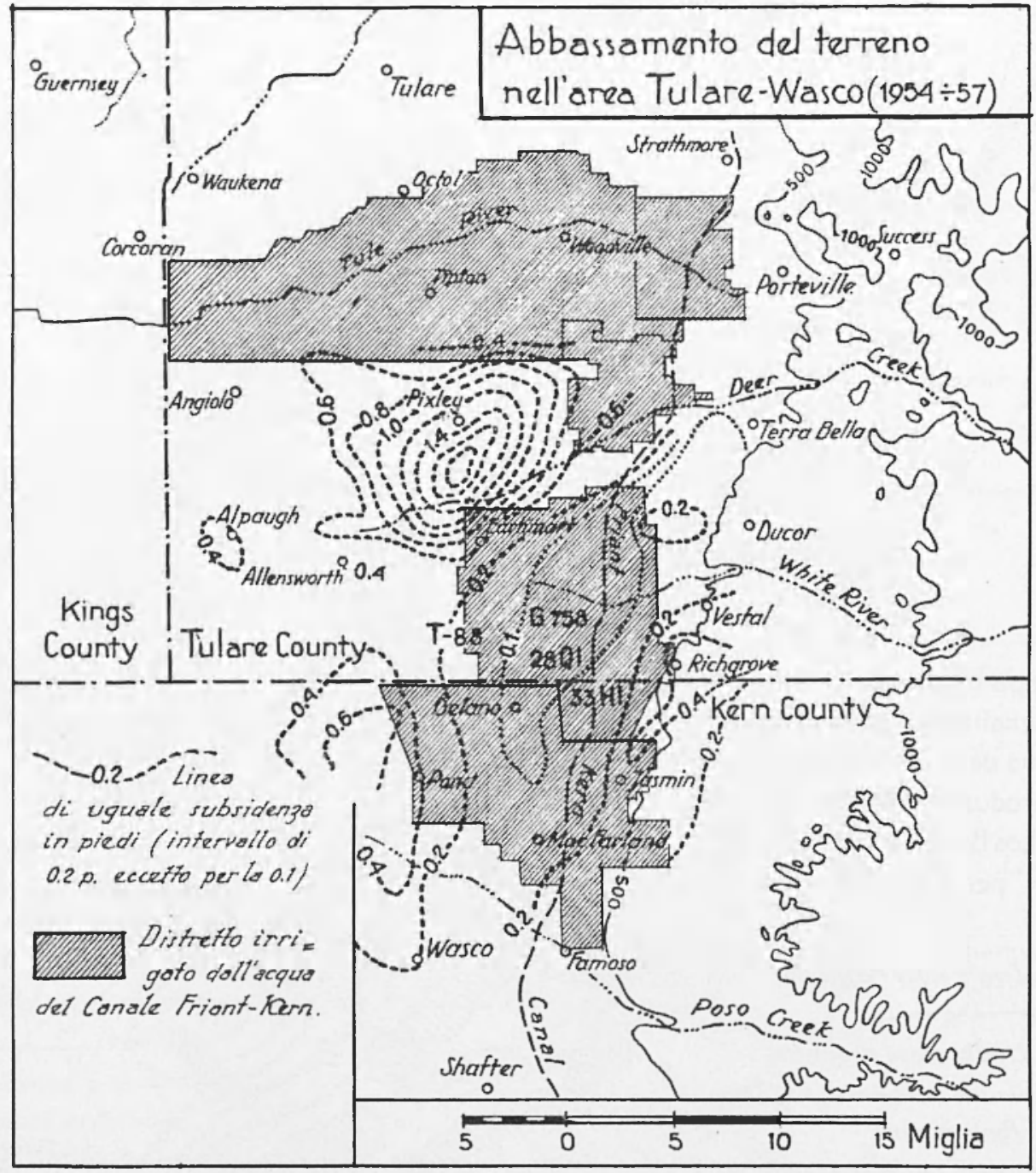

Fig. 11 - Abbassamento del terreno nel distretto Tulare-Wasco, dal 1954 al 1957 (secondo l'I.A.C.).

del bacino della valle ("No evidence of shallow subsidence has been observed on either the old alluvial - fan soils or the soils of the valley basin ", pag. 87). 


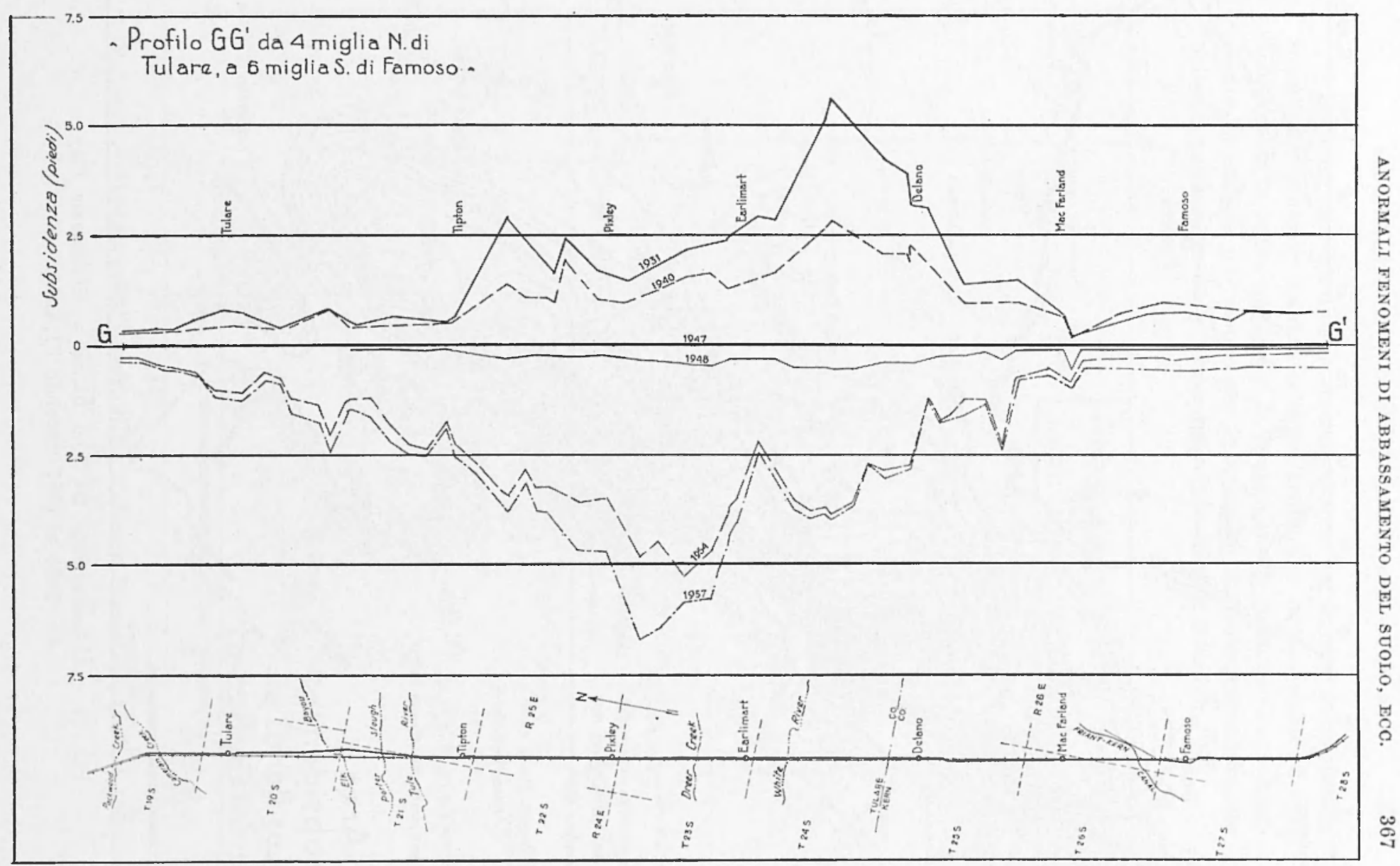

Fig. $11 a$ - Particolari dell'abbassamento nella zona Tulare-Famoso, dal 1931 al 1957 (secondo l'I.A.C.). 


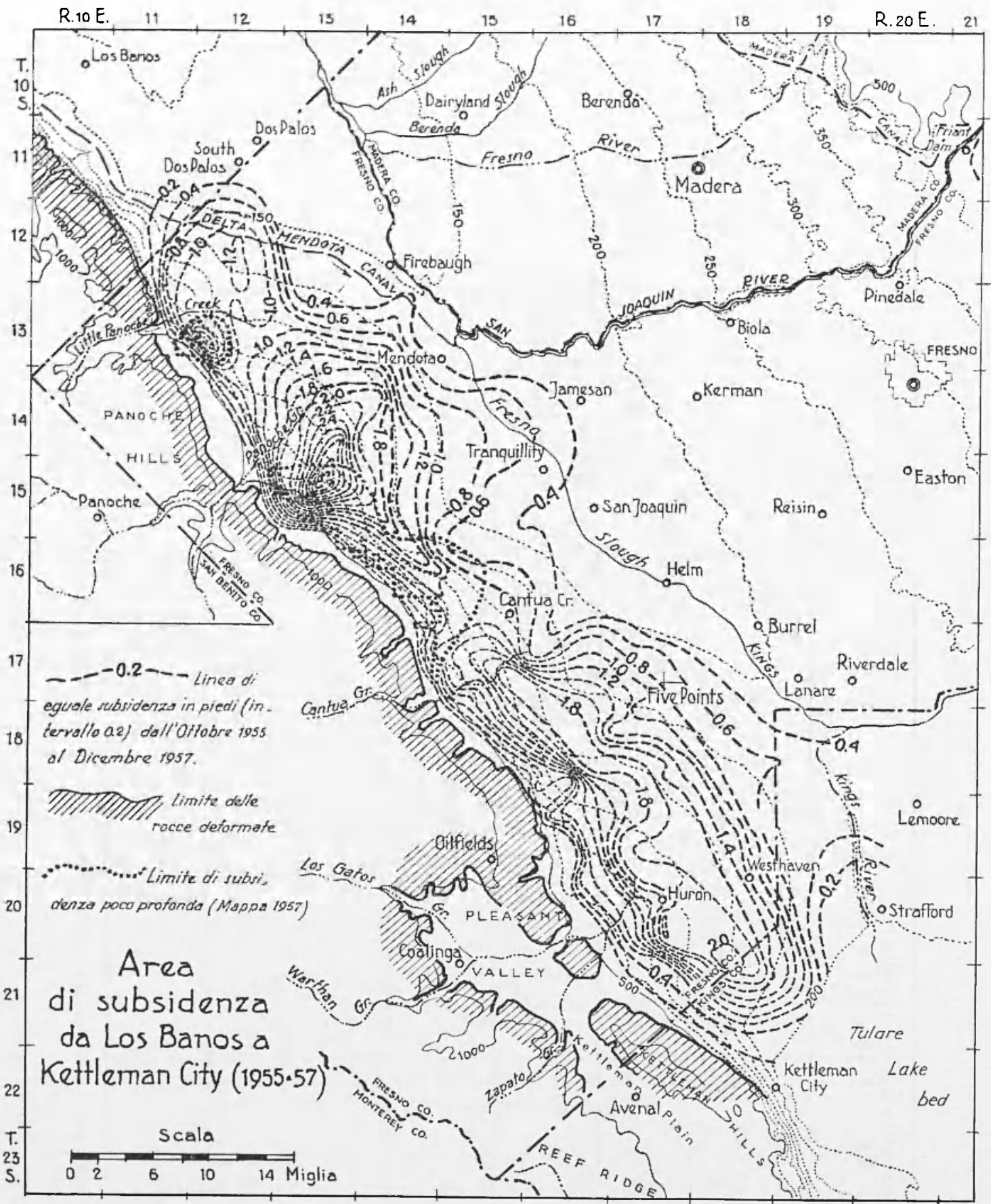

Fig. 12 - Abbassamento fra Los Banos e Kettleman City dal 1955 al 1957 (secondo l'I.A.C.). 
Depositi alluvionali che si comprimono in seguito a irrigazione sono tipicamente di umidità relativa bassa e di bassa densità. I dati concernenti la bassa umidità e la bassa densità costituiscono, attualmente, il criterio più idoneo per individuare depositi potenzialmente flettenti nella parte occidentale della valle di San Joaquin.

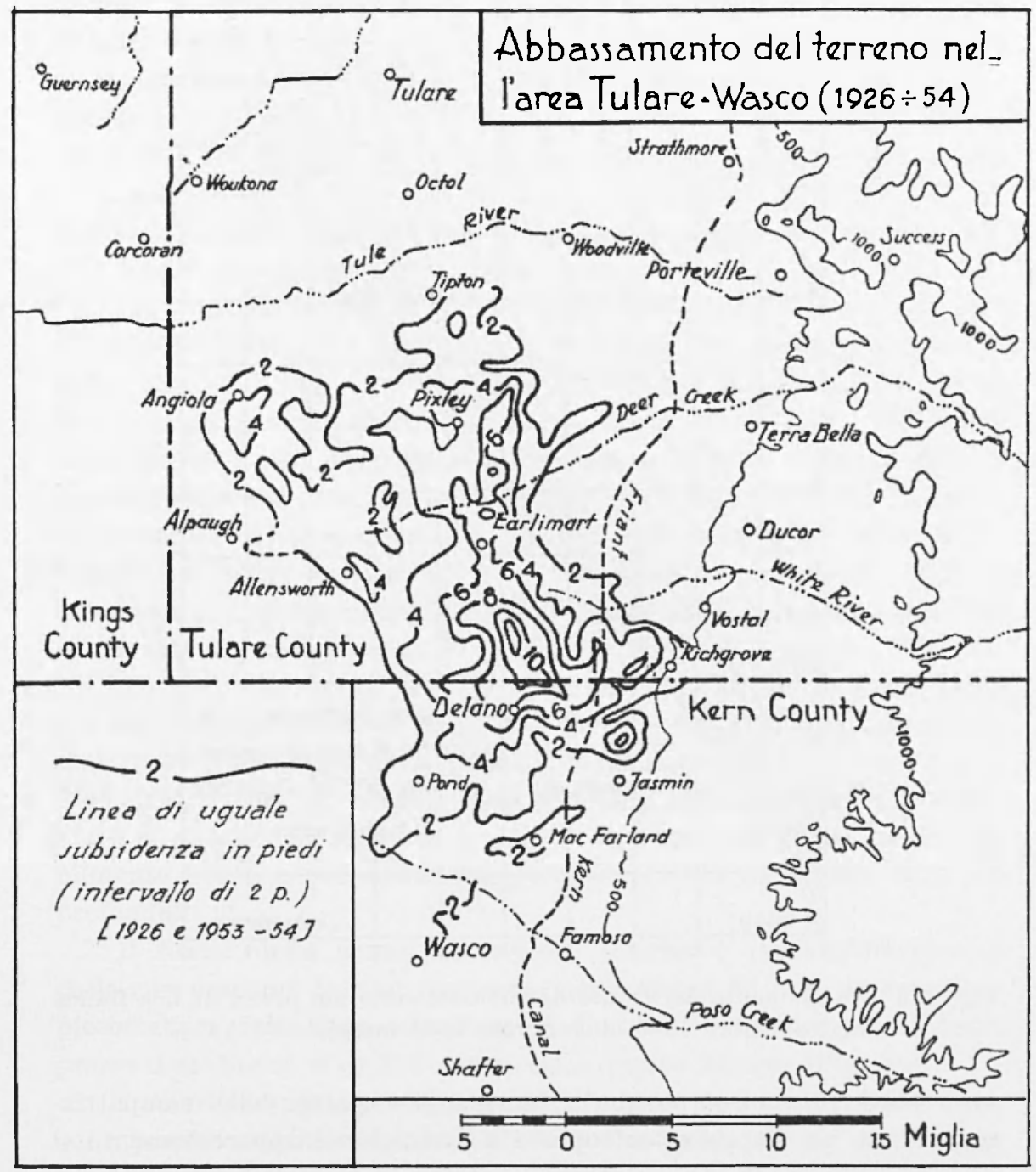

Fig. 12a - Abbassamento osservato fra Tulare e Wasco dal 1926 al 1954 (secondo l'I.A.C.). 
La flcssione è strettamente connessa alla variazione che si produce nelle caratteristiche di un deposito, quando viene irrigato. Diminuzione di volume e aumento di densità accompagnano l'abbassamento. Apparentemente, mai dopo la loro formazione tali sedimenti

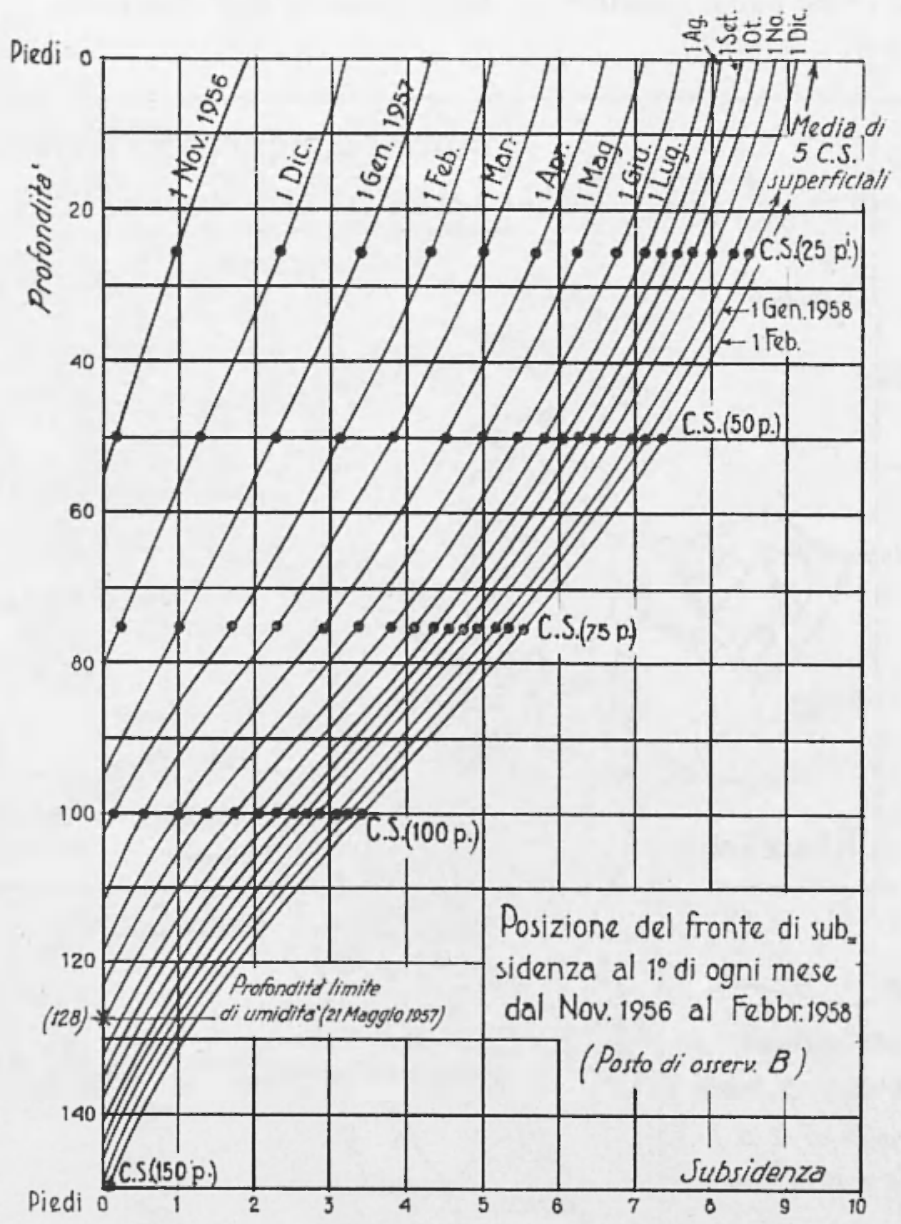

Fig. $12 b$ - Andamento del fronte di abbassamento nei pressi di Los Banos dal Novembre 1956 al Febbraio 1958 (secondo l'I.A.C.).

sono stati interamente bagnati. La maggior parte della compattizzazione di un deposito, sottoposto a irrigazione, apparentemente si verifica all'immediato contatto, e subito dopo, col fronte d'acqua avanzante. Questo tipo di abbassamento avviene soltanto in depositi sopra la falda freatica (pag. 87). 
2) Abbassamento in profondità. - Nella valle di San Joaquin furono praticati pozzi per estrazione di acque ad uso domestico prima del 1870; però, le acque sotterranee non furono usate estensivamente per irrigazione fino al periodo della $1^{a}$ guerra mondiale. L'estrazione di acque sotterranee per irrigazione procedette a ritmo moderato dal 1915 al 1929; il pompaggio annuo per il $1929 \mathrm{fu}$ stimato essere dell'ordine di $123.400 .000 \mathrm{~m}^{3}$. Per il 1937 il pompaggio fu di 287.522 .000 $\mathrm{m}^{3}$ /anno e per il 1944 aumentò a $435.602 .000 \mathrm{~m}^{3}$. L'estrazione di acque sotterranee crebbe nella parte centro-occidentale della valle, stimolata dagli alti prezzi del raccolto, durante gli anni dal 1945 al 1953. Dai $435.602 .000 \mathrm{~m}^{3}$ del 1944 , il pompaggio annuo crebbe più di cinque volte tanto, raggiungendo $2.313 .750 .000 \mathrm{~m}^{3}$ nel 1953. A causa della diminuita superficie di produzione agricola, specialmente del cotone, il pompaggio decrebbe nel 1954 e nel 1955 (Fig. 13).

La zona dei depositi di "fresh-water-bearing" dell'area di Los Banos-Kettleman City può essere divisa in una parte superiore di "silt, clay and sand", principalmente depositi alluvionali ed eolici, che si estende dalla superficie esterna alla profondità da 200 a 800 piedi (da 60 a 240 metri circa); una parte intermedia (" of impervious diatomaceous clay, the Corcoran clay member of the Tulare formation, of lacustrine origine ") spessa da 20 a 120 piedi (da 6 a $36 \mathrm{~m}$ circa); e da una parte inferiore (" of silt clay and sand, in part of lacustrine origin "), comunemente spessa da 600 a 1500 piedi (da 180 metri a 450 circa), ma localmente di uno spessore di 3000 piedi (900 metri circa).

Un corpo di acqua semi-imprigionata occupa la maggior parte del settore superiore; la profondità dell'acqua si estende fra meno di 3 metri a 90 metri circa sotto la superficie esterna. Le acque profonde nel settore più basso, sono confinate nella maggior parte dell'area a ghiaie del Corcoran e sono sotto pressione artesiana. Probabilmente $1 ' 80 \%$ o più dell'acqua pompata deriva da questa zona più profonda.

Il limite delle acque nel settore esterno è mutato lievemente dalle sue posizioni iniziali; tuttavia, negli ultimi 50 anni la superficie piezometrica della zona sottostante è diminuita (decaduta) di 20 metri presso Los Banos e di 120 metri circa presso Huron (Fig. 10).

I rilievi compiuti nel 1935 dall'"U.S. Coast and Geodetic Survey ", sui caposaldi stabiliti nel 1919 dall'" U.S. Geological Survey " indicarono un declino di quota. Le misure di livellazione ripetute nel 1943, sulle linee di livello stabilite nel 1935 confermarono che l'abbassamento interessava una vasta zona. La Fig. 9, relativa alla flessione per 


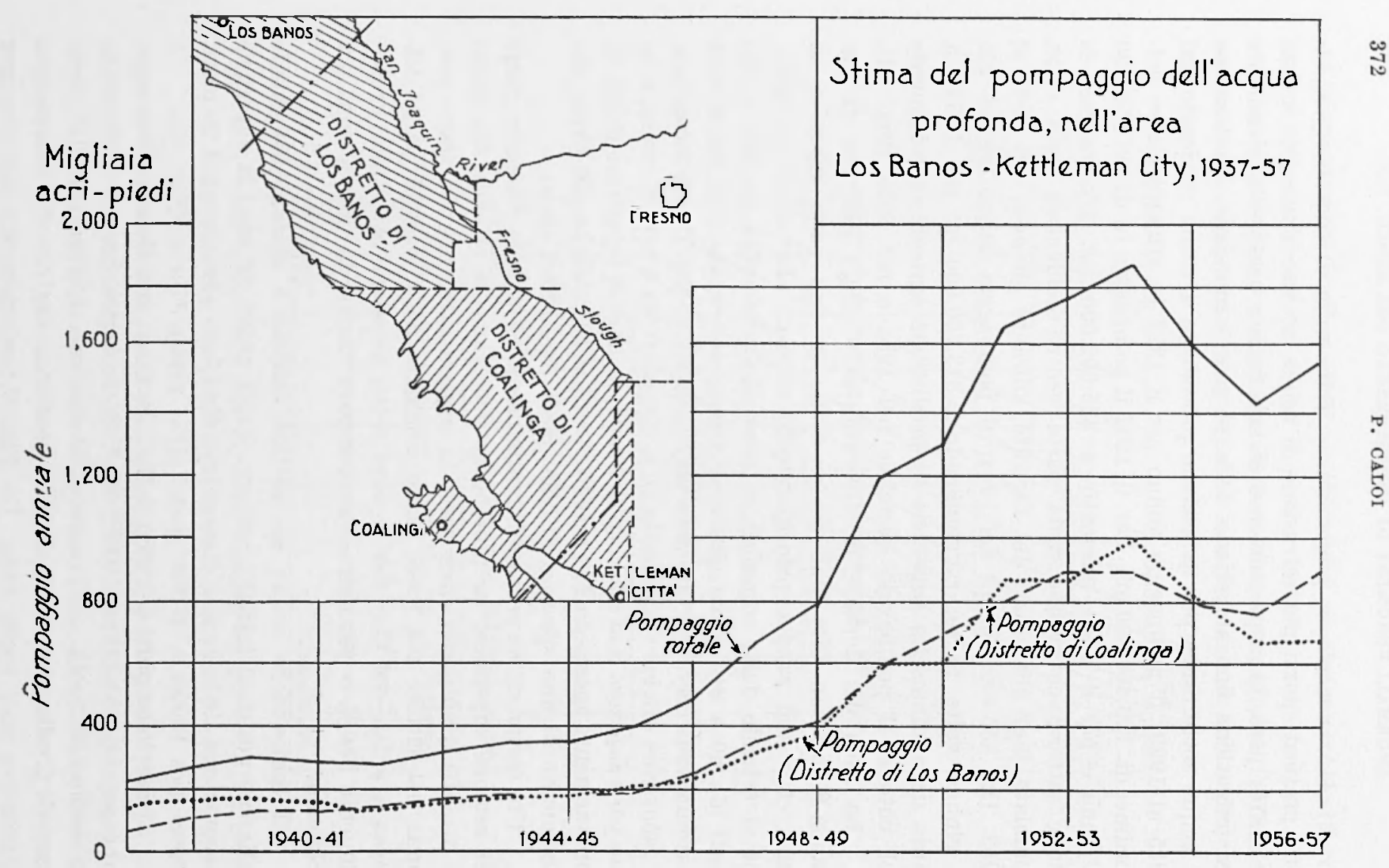

Fig. 13 - Estrazione di acque profonde nell'area Los Banos-Coalinga dal 1937 al 1957 (secondo l'I.A.C.). 
il periodo 1943-1953, indica un abbassamento di più di $30 \mathrm{~cm}$ per un'area di approssimativamente $2.700 \mathrm{~km}^{2}$.

Sulla base degli studi idrologici, già dal 1956 Poland e Davis ( ${ }^{17}$ ) conclusero che l'esteso abbassarsi del livello delle acque sotterranee era da ritenersi come una delle cause primarie, se non la causa principale, della flessione nell'area Los Banos-Kettleman City. In breve, il declino del livello delle acque serve a rimuovere parte del supporto idraulico dei materiali sovrastanti e, perciò, equivale all'aggiunta di corrispondente carico ai depositi dei bacini coperti (pag. 114). E nel 1958 si conferma: "Analysis of the occurrence, movement, and especially the fluctuations of the ground water is essential in determining the past and present loading of the deposits, in evaluating differences in compaction in deposits of differing lithology, and in predicting future subsidence " (pag. 114).

Nel lavoro di Poland sono riportati numerosi dati tecnici [234 idrografi di pozzi d'irrigazione, grafici di parecchi registratori automatici di livello, ecc., superficie piezometriche, ecc. (pagg. 114115-116)].

Esperimenti di laboratorio furono eseguiti in gran copia.

Porosità e rapporto di vuoto, umidità, contrazione ecc. (pag. 132 e successive) sono state rilevate per diversi esemplari dei settori superiore e inferiore. Com'era da attendersi, dato il diverso carico, i depositi del settore superiore risultarono generalmente più porosi di quelli del settore sottostante, sebbene la differenza non sia notevole.

Il rapporto di vuoto $\left(\frac{n}{1-n}, n\right.$ porosità, in percento di volume) per campioni del settore superiore supera 0,7 , variando fra 0,7 e 0,9 ; in molti campioni nel settore inferiore è minore di 0,7 , variando fra 0,5 e 0,7 .

I limiti di contrazione risultarono fra 20 e 30 per cento (pag. 134).

La gran parte dell'acqua pompata proviene dai depositi imprigionati nel settore inferiore e le diminuzioni di livello si son verificate principalmente in questa zona ( (lower unit»): Figg. 14, 15, 9. La Fig. 13 mostra il grafico del pompaggio nell'area fra Los Banos e Kettleman City per il periodo 1937-57.

L'enorme progresso ("the tremendous growth") del pompaggio nell'area Los Banos-Kettleman City, specialmente dal 1945, ha avuto effetto determinante sulla superficie piezometrica dei depositi imprigionati nella zona d'acqua inferiore e fu la causa prima del declino del livello nell'area. Dal 1944-45 al 1953-54, anno di massima estra- 


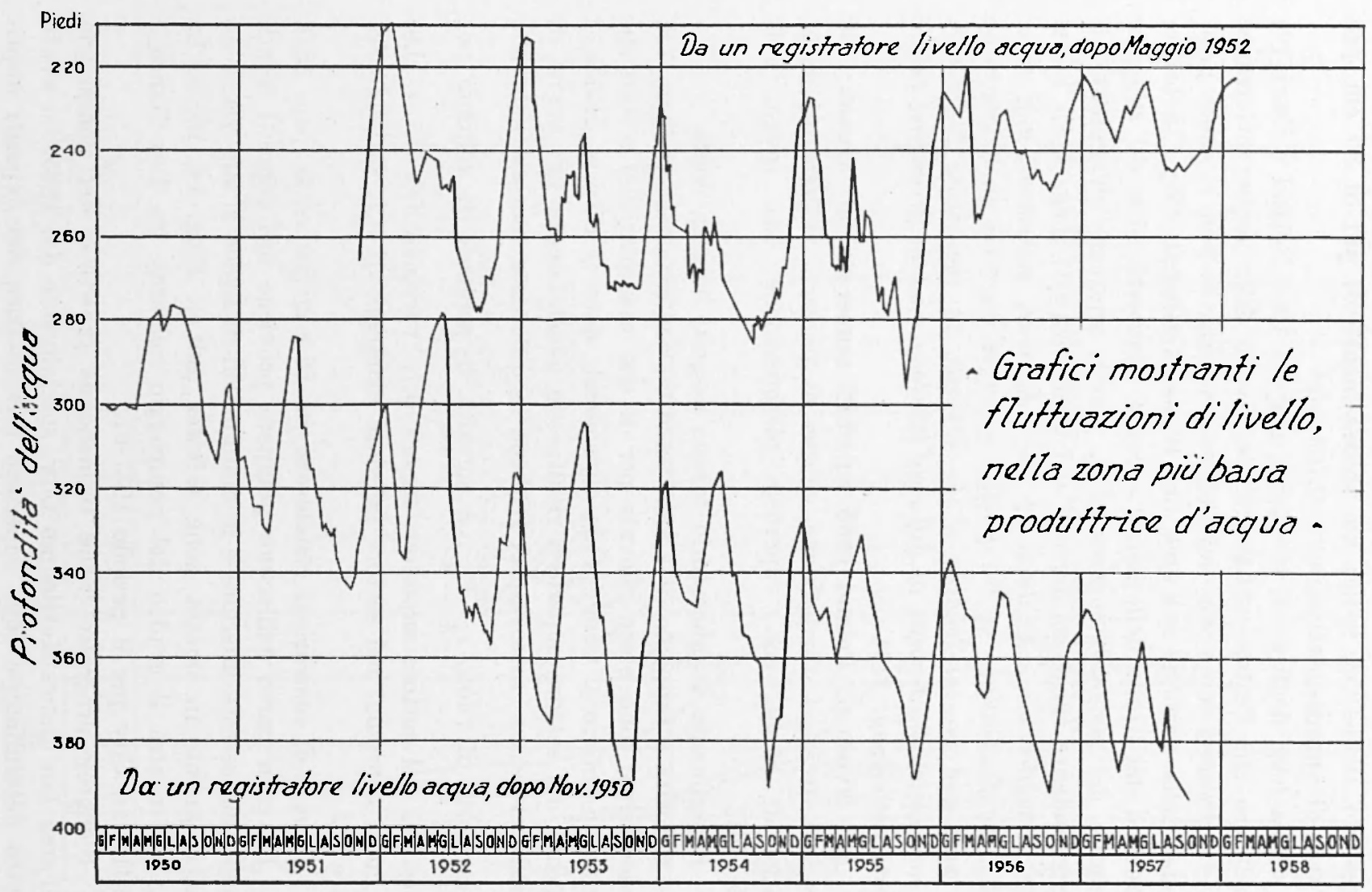

Fig. 14 - Fluttuazioni nelle acque profonde, in due zone diverse (secondo l'I.A.C.). 
zione, il pompaggio divenne cinque volte maggiore passando da circa 350.000 " acre-feet " a circa 1.875 .000 " acre-feet".

La Fig. 14 mostra le fluttuazioni stagionali del livello artesiano nella zona d'acqua d'estrazione inferiore, oltre alle tendenze a più lunga scadenza. Essa delinea una dettagliata registrazione del carico e scarico idraulico dei depositi sotterranei, come conseguenza de declino e della ripresa del livello.

"Land-surface subsidence caused by decline in fluid pressure in confined systems has long been known from experience in several areas of heavy ground-water draft and from oil fields in several widely separated places ${ }^{17}$ ) (pag. 144).

Fra i risultati di massimo interesse raggiunti dal Comitato di studio della "Land Subsidence in the San Joaquin Valley " va richiamato il seguente, che può essere riportato di peso al caso del Delta padano: "Comparison of changes in head to subsidence show that even locally the ratio of change in head to subsidence differs considerably. The causes of these differences appear to be chiefly geologic-such factors as differences in mineral composition, sorting, degree of consolidation, degree of cementation, and degree of confinement of the deposits. These factors generally are related to the geology of the source areas, environment of deposition, and postdepositional history of the deposits. Furthermore, hydrologic factors also may be important, such as vertical pressure gradients in the confined deposits and time lag in compaction of the materials related to slow drainage of fine-grained deposits of low permeability " (pagg. 144-145).

Nella zona Los Banos-Kettleman City Area, l'area affetta da flessione (Fig. 16) copre circa 1100 square miles (pari a circa $2860 \mathrm{~km}^{2}$ ).

L'area di abbassamento ha la forma di un ovale, lungo e stretto. I centri di massima flessione sono ad ovest di Mendota (più di 18 piedi $=5,50 \mathrm{~m}$ da 6 a 8 miglia ad occidente di Mendota) e a nord di Huron (più di 14 piedi $=4,3 \mathrm{ml})$. L'area di flessione di 4 piedi $(1,2 \mathrm{~m})$ o più abbraccia 480.000 acri (pari a 190.000 ettari); quella di 8 piedi o più $(2,4 \mathrm{~m}), 150.000$ acri (pari a 60.000 ettari); e quella di 10 piedi (3 m) o più, 65.000 acri (26.000 ettari). Quest'area (Fig. 16) comprende la "shallow " e la "deep subsidence " sopra e sotto la "water table" (Figg. 17, 18, 19, 20, 21, 22, 23, 25).

L'U.S.C.G.S. rilevò la zona due volte, recentemente (SettembreNovembre 1955 e Novembre-Gennaio 1957-58). La massima flessione nei 26 mesi circa d'intervallo fra l'una e l'altra campagna fu osser- 
vata 12 miglia a SE di Mendota e fu di 3,5 piedi (m 1 circa). Un massimo secondario di 2 piedi $(60 \mathrm{~cm}$ ea) si verificò 6 miglia a SE di Huron Figg. 12.

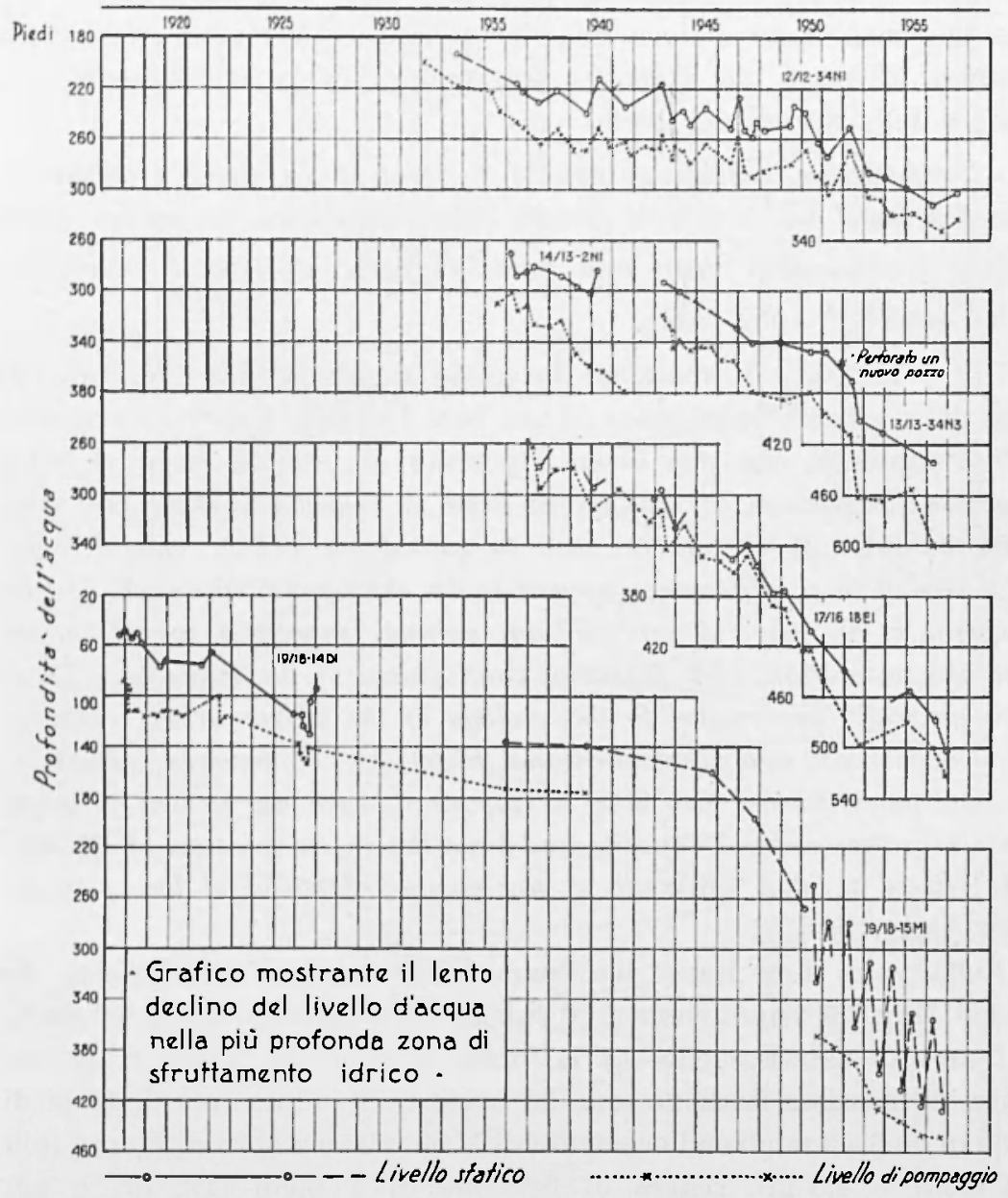

Fig. 15 - Altri esempi di declino delle acque profonde per estrazione (secondo l'I.A.C.).

Il confronto fra le Figg. 12 e 16 (flessione fra 1922-32 e fino al 1956) mostra che il centro settentrionale della flessione ora è emigrato 8 miglia S-SW, apparentemente a causa della forte influenza della "shallow subsidence" e di un progressivo aumento del pompaggio. Per la compressione, vedi Fig. 24. 


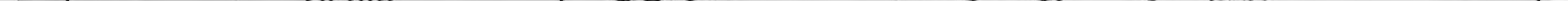




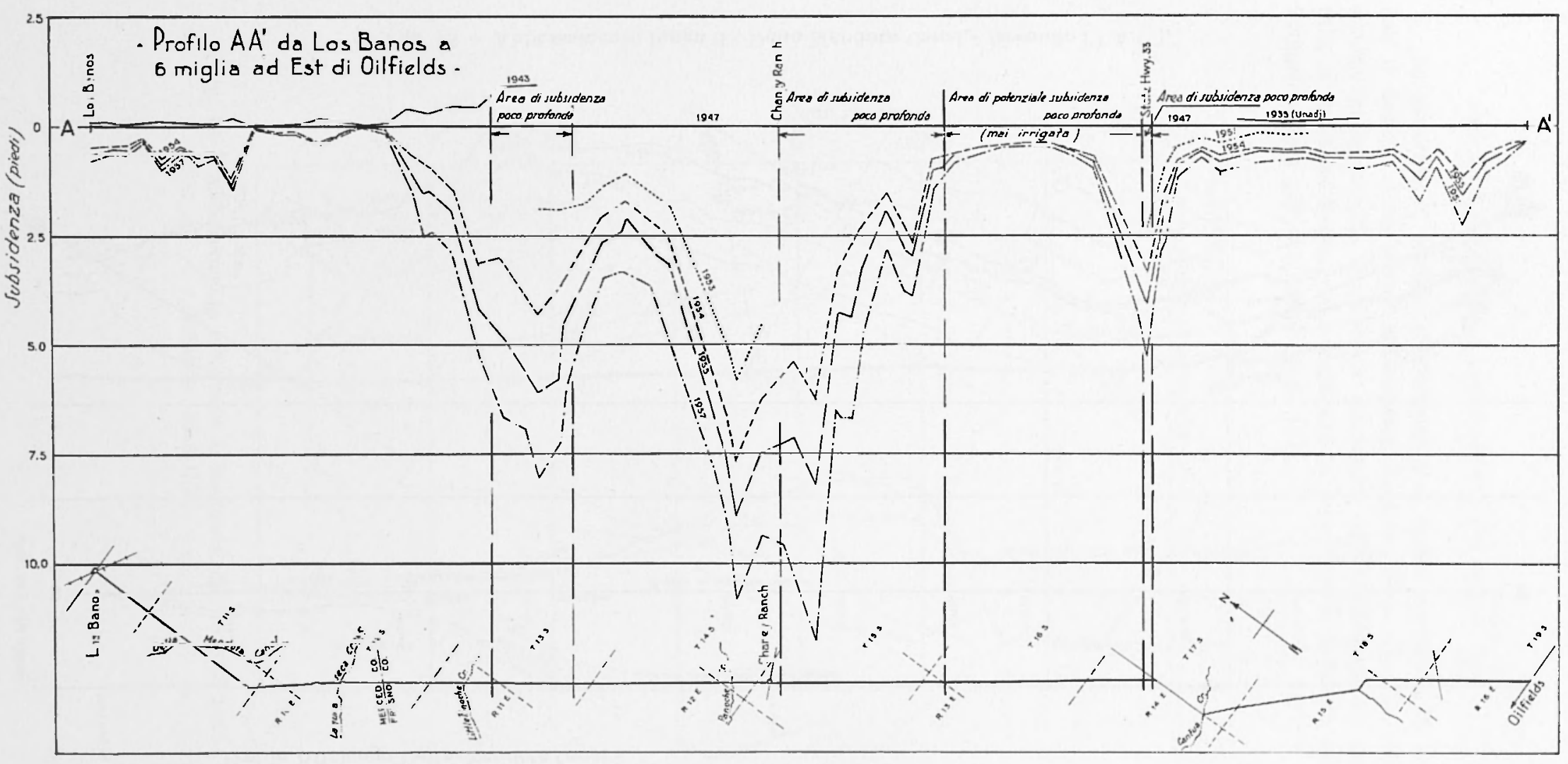

Fig. 17. - Abbassamenti del terreno fra Los Banos e Oilfields (secondo l'I.A.C.). 

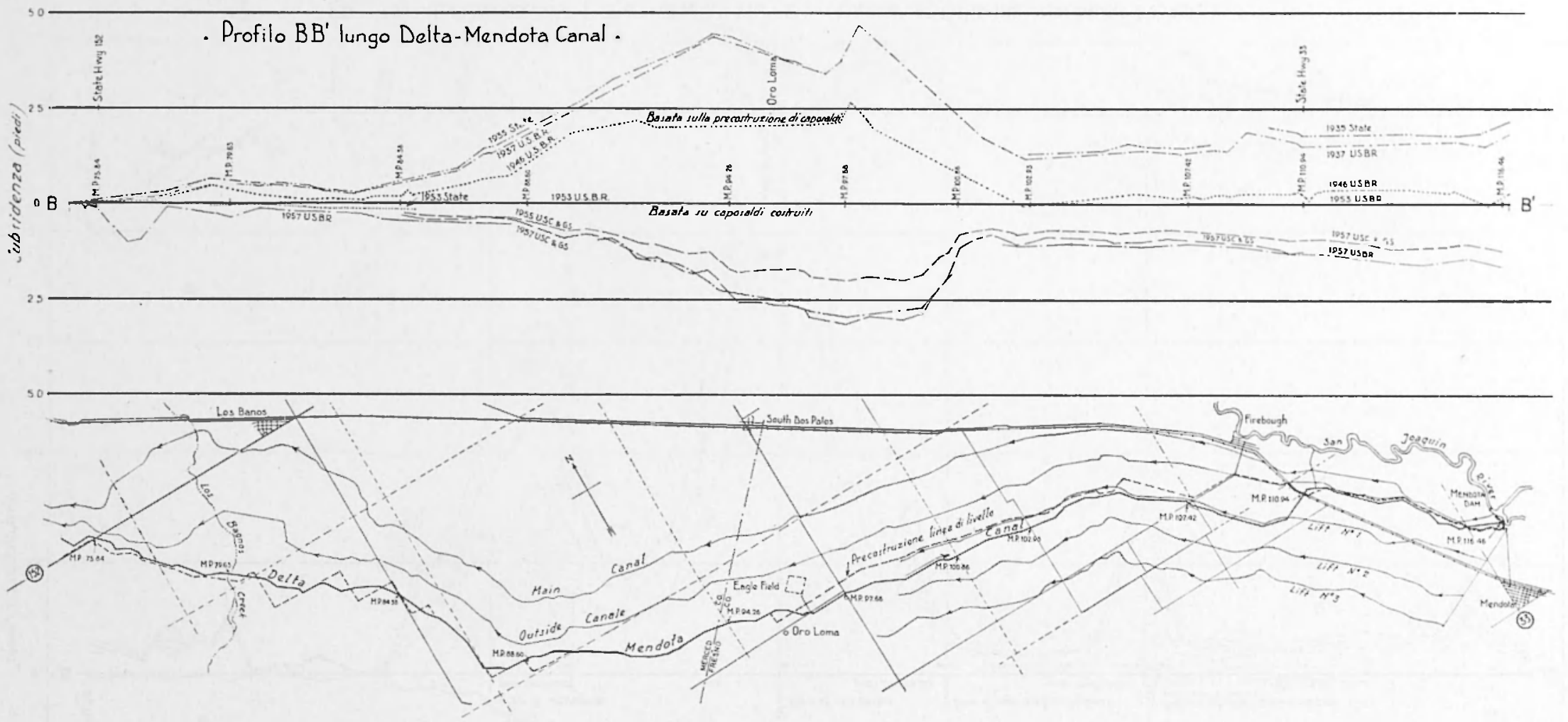

Fig. 18 - Abbassamento lungo il "Delta Mendota Canal (secondo l'I.A.C.). 
In generale, il terreno in flessione può essere correlato, in area, con il declino delle acque sotterranee. Inoltre (Figg. 17, 21), esiste stretta relazione fra flessione e progresso dell'irrigazione: in generale, la massima flessione si è verificata in aree che sono state sotto irrigazione per parecchi anni (pag. 147).

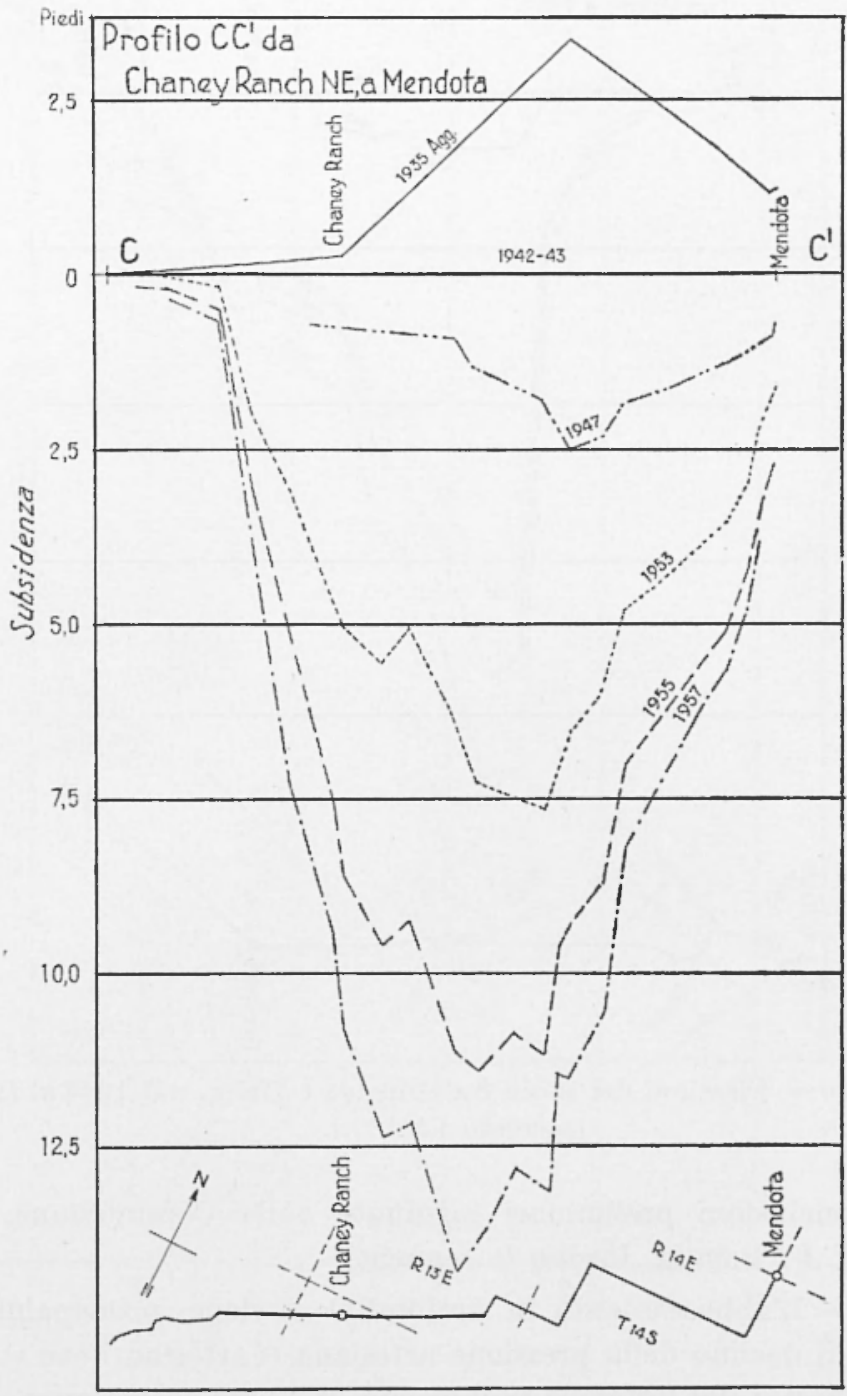

Fig. 19 - Abbassamento nei pressi di Mendota, dal 1935 al 1957 (secondo I'I.A.C.). 
Altre coincidenze significative: l'entità della flessione è stata accelerata negli ultimi anni, "which is not surprising considering the increases in pumping in the area (Fig. 13) and the steepening of decline of water levels» (Figg. 14, 15) (pag. 147).

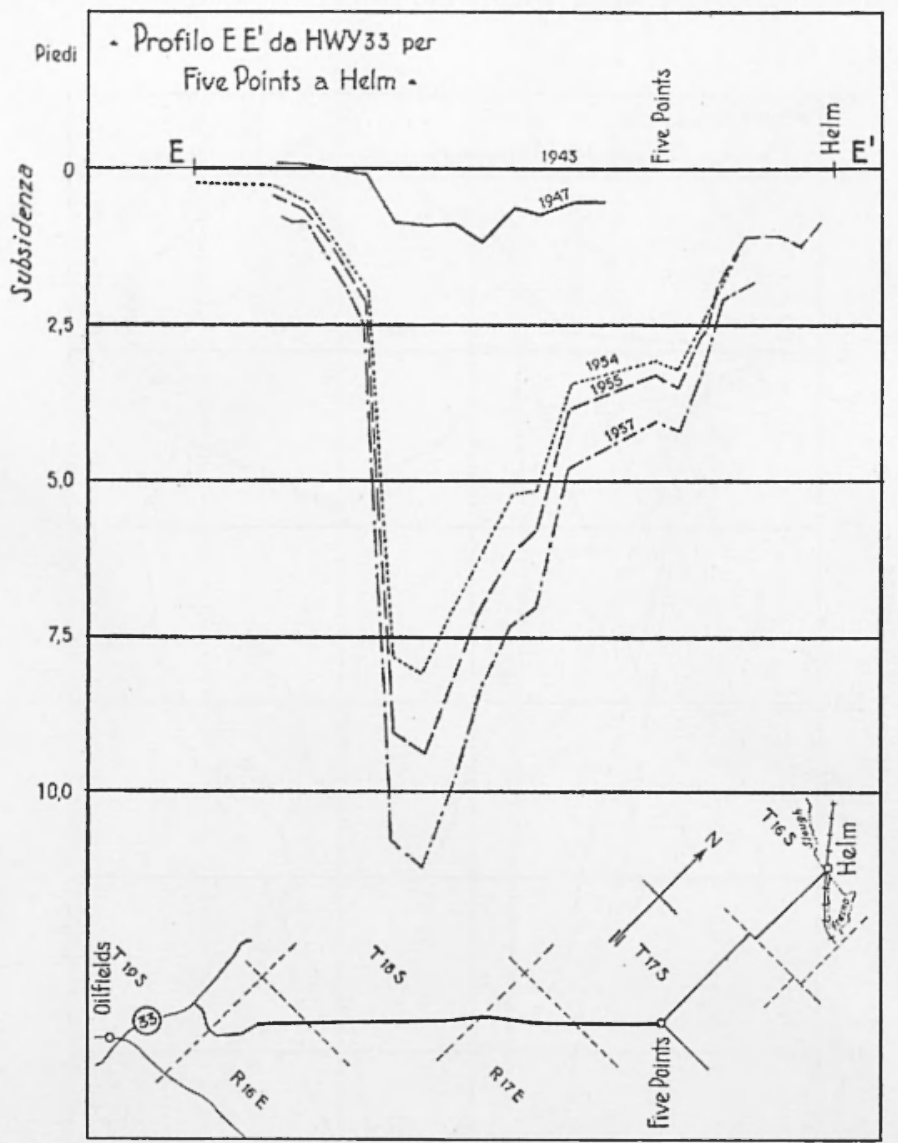

Fig. 20 - Flessioni del suolo fra Oilfields e Helm, dal 1943 al 1957 (secondo l'I.A.C.).

Le conclusioni preliminari raggiunte dalla Commissione, presieduta da J. F. Poland, furono le seguenti:

a) - L'abbassamento in profondità avviene principalmente in aree dove il declino della pressione artesiana (" artesian head ") è stato grande, a causa del notevole pompaggio di acque sotterranee. Inoltre, i maggiori mutamenti nella distribuzione del pompaggio si sono riflessi in mutamenti nella distribuzione dell'abbassamento. 


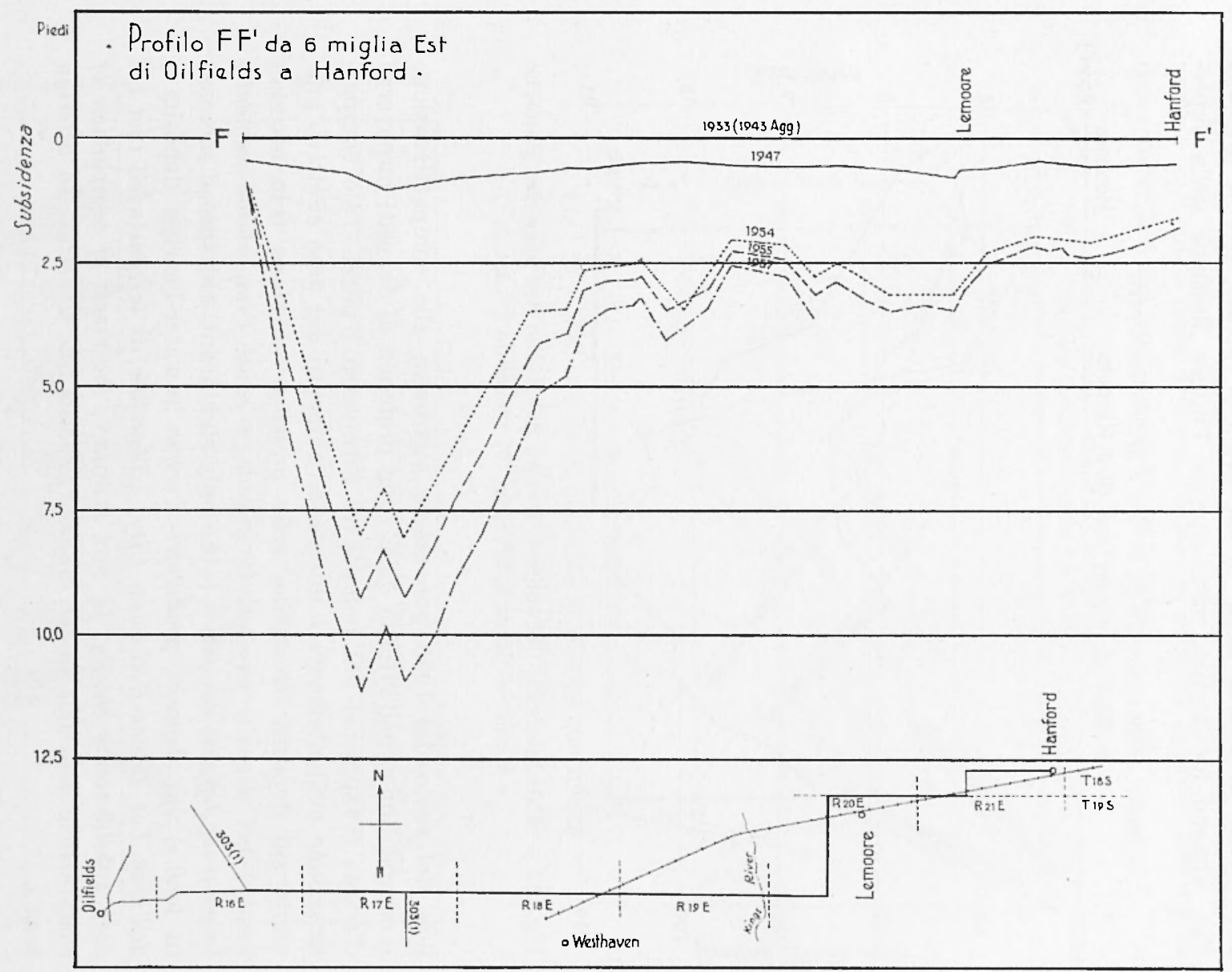

Fig. 21 - Flessioni del suolo fra Oilfields e Hanford, dal 1933 al 1957 (secondo I'I.A.C.). 
b) - Le regristrazioni ad un caposaldo profondo ancorato con cavo ("anchoredcable deep bench mark"), presso Huron indicano che, almeno localmente, virtualmente l'intera flessione della super-

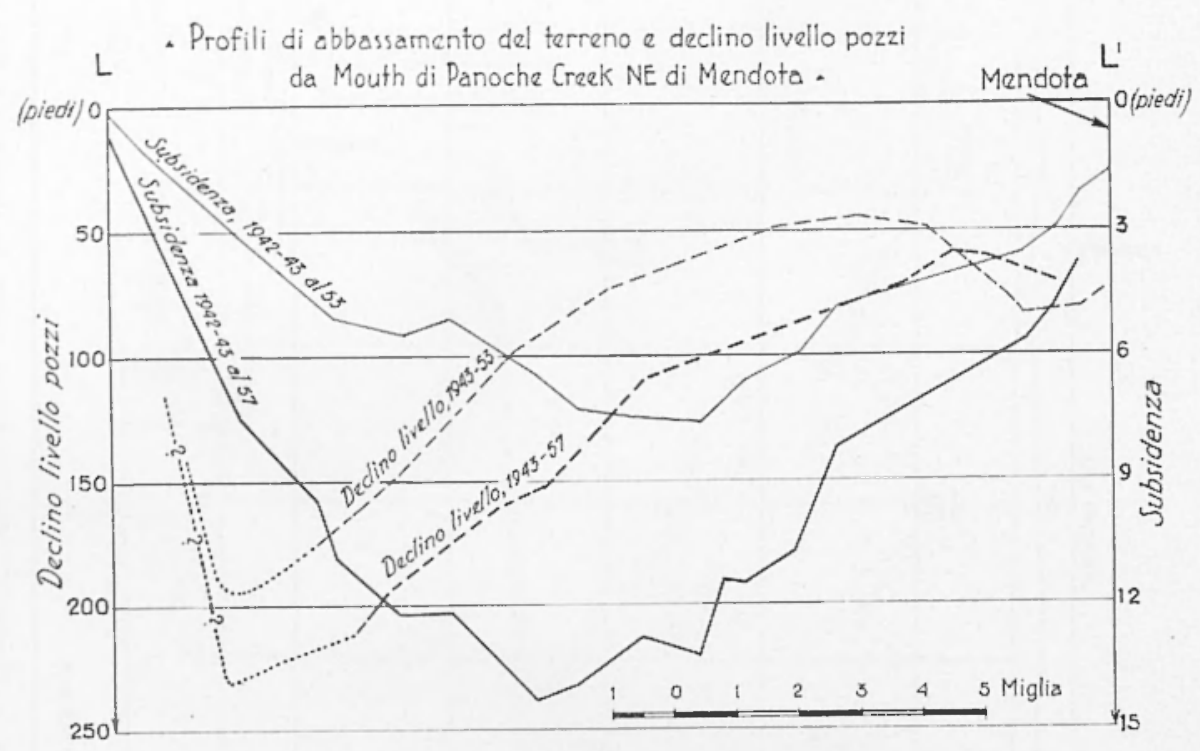

Fig. 22 - $\Lambda$ bbassamenti del suolo e declino del livello dei pozzi fra Panoche e Mendota (linea L-L', Fig. 9) (secondo l'I.A.C.).

ficie del suolo dal 1955 può essere attribuita alla compattizzazione (" compaction ") nell'interno della zona profonda di depositi impoveriti d'acqua ("tapped") dal pompaggio attraverso i pozzi. Tale compattizzazione evidentemente è dovuta all'aumento del peso effettivo gravante sui depositi. Il declino nella pressione artesiana ("in artesian head ") nei depositi nascosti fu grande, e causò l'importante aumento, largamente diffuso, del carico (" the only significant widespread increase in load") sui depositi produttori d'acqua ("water-bearing deposits") dell'area Los Banos-Kettleman City. "Therefore, it is concluded that the deep subsidence is chiefly, IF NOT WHOLLY, the result of compaction of compressible materials in the confined deposits due to decline in artesian head n.

c) - Il confronto delle registrazioni dei caposaldi e dei misuratori di livello delle acque indica che vi è poco o nessun ritardo fra applicazioni del carico e flessione della superficie del suolo. Infatti, i risultati di un registratore di abbassamento (Fig. 26) presso Huron 
indicano che - sotto condizione che vi esista - la flessione è stagionale, verificandosi durante i periodi di più marcato declino del livello d'acqua; la ripresa di quest'ultimo conduce, ad una diminuzione o arresto nell'entità della flessione. Tuttavia, nessuna apprezzabile ascesa della superficie del suolo è stata osservata.

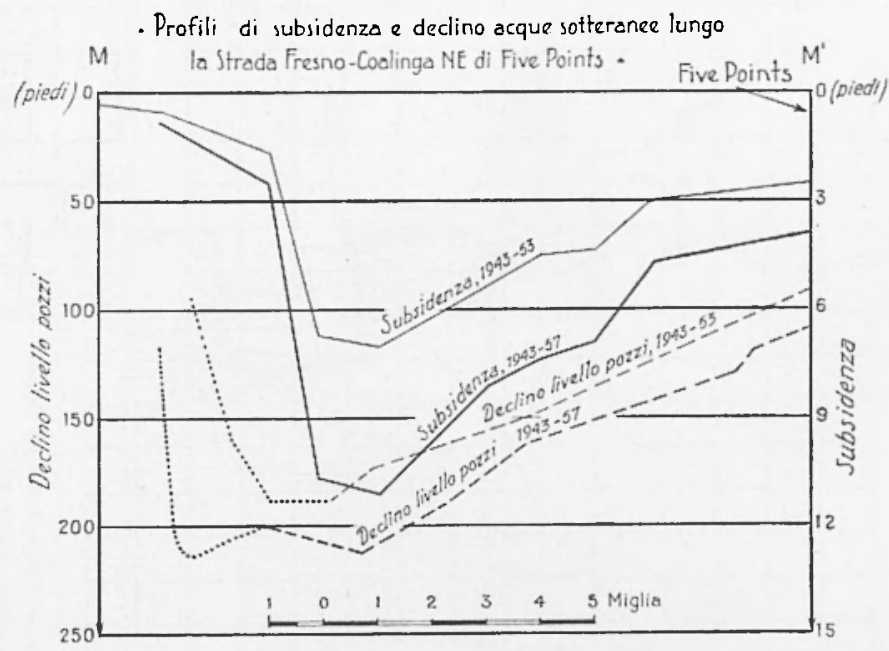

Fig. 23 - Abbassamenti del suolo e declino del livello dei pozzi fra Oilfields e Five Points (linea M-MI', Fig. 9), dal 1943 al 1957 (secondo l'I.A.C.).

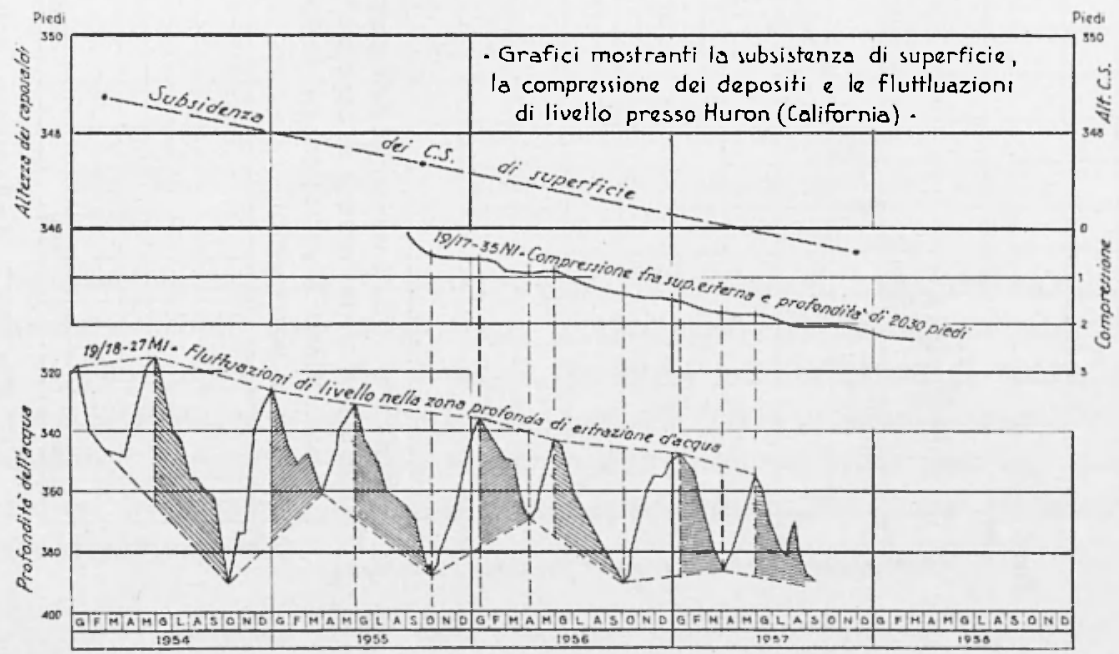

Fig. 24 - Flessione, compressione dei depositi e fluttuazioni di livello presso Huron (secondo l'I.A.C.). 


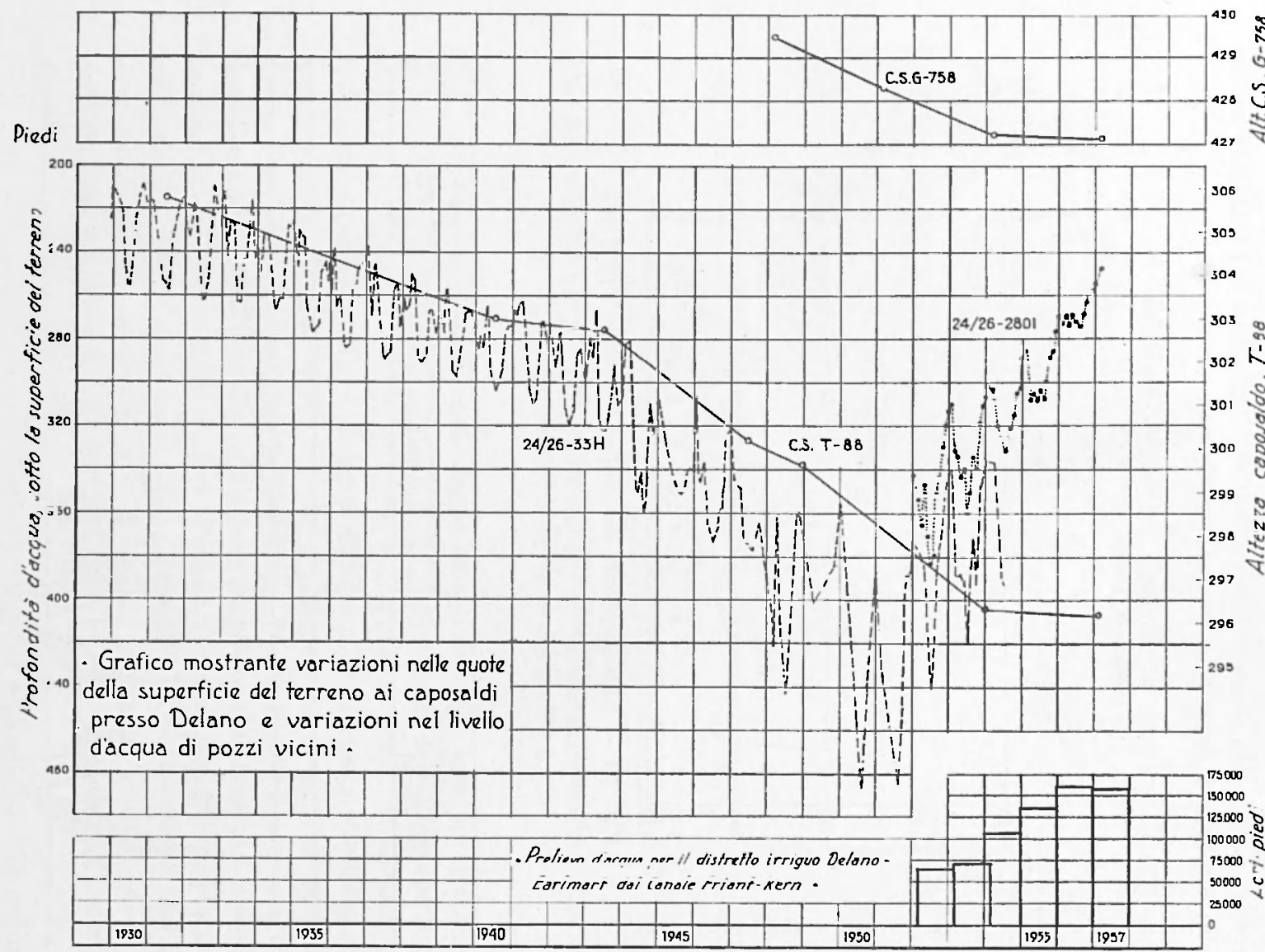

Fig. 25 - Andamento della flessione del terreno e variazioni del livello dell'acqua nei pozzi, nei pressi di Delano (secondo l'I.A.C.). 
4. - "Compaction occurs chiefly in poorly consolidated alluvial and lacustrine deposits of Pliocene and Pleistocene age ". In laboratorio, $\mathrm{i}$ materiali piu compressibili sono le fortemente porose argille (" clays ")

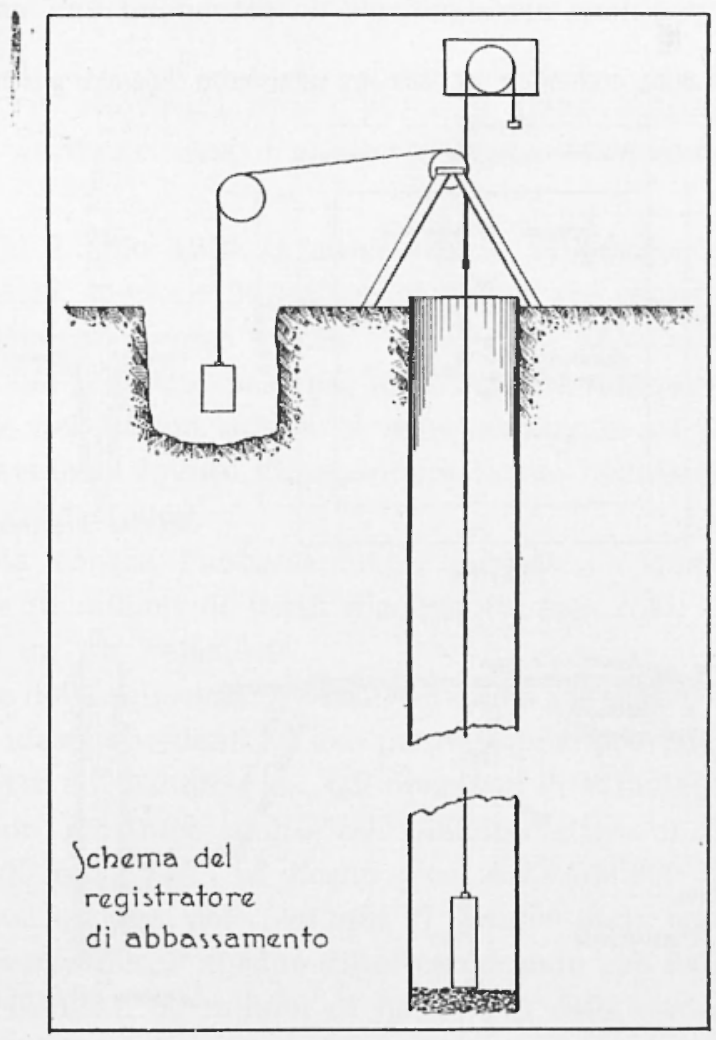

Fig. 26 - Schema di un registratore di flessione (secondo l'I.A.C.).

lacustri, "benché le argille sedimentose (" silty clays "), i limi ("silts ") ed anche le sabbie mostrino di essere compressibili entro una certa misura.

Comunque, c'è una generale tendenza per un grado di naturale consolidamento ("consolidation "), crescente con la profondità (pag. 152). Physical properties that accompany high compressibility are high porosity, low density, and high liquid limit, plastic limit, and shrinkage (contrazione) limit.

5. - A causa delle differenze geologiche, l'andamento della flessione in relazione al declino nel livello delle acque sotterranee (" in head") NON È COSTANTE attraverso l'area. Comunque, predizioni di abbassa- 
mento appaiono possibili se sono utilizzabili sufficienti dati geologici e idrologici.

Come conclusione, i relatori osservano che, continuando la diminuzione nella pressione artesiana, c'è da attendersi una continuazione Schema apparecchiatura, per osservare subsistenza caposaldo profondo
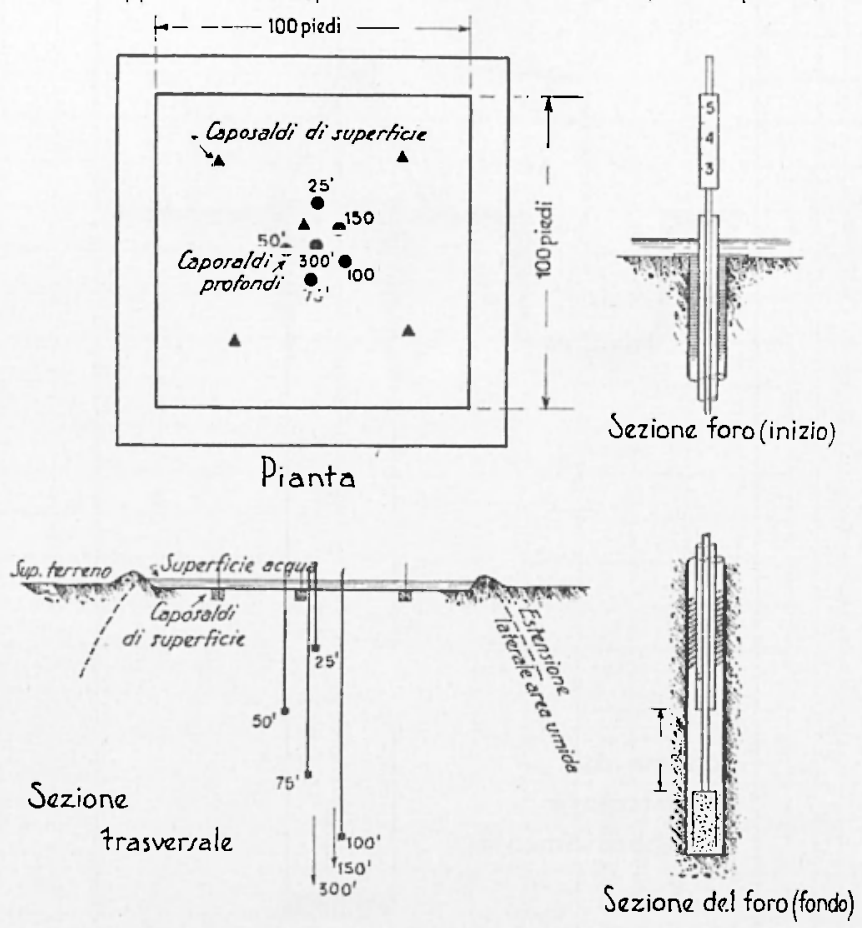

Fig. 27 - Schema di apparecchiatura per l'osservazione di abbassamento in profondità (secondo l'I.A.C.).

dell'abbassamento. Esperienze compiute nell'area Tulare-Wasco indicano che, se vengono immesse acque in quantità sufficiente da ristabilire all'incirca $i$ precedenti livelli, la flessione profonda viene rallentata $e$ pud essere arrestata.

In conclusione, ritengo superfluo sottolineare l'estremo interesse dei fenomeni di abbassamento osservati nella San Joaquin Valley, in zone più estese di quelle del Delta padano e in condizioni di evidente analogia. Ciò sta a convalidare l'attendibilità delle deduzioni, con cui termina la mia relazione sull'abbassamento del Delta padano.

Roma, Giugno 1959. 


\section{$\triangle P P E N D I C E$ II}

ABbassamenti aNormali Nella zoNa di LONG BeacII E PROVVediMENTI ADOTTATI PER L'ARRESTO DELLA FLESSIONE IN ATTO (*).

1. - Nel Luglio 1959 la situazione a Wilmington (Long Beach) era la seguente: spesi già 90 milioni di dollari per lavori di riparazione. Se l'area depressa avesse subito un ulteriore abbassamento di $10 \circ$ 15 piedi, erano previste spese per altri 50 o 60 milioni di dollari; e se la superficie del bacino navale si fosse abbassata di più di 4 piedi, la Marina avrebbe dovuto abbandonare le sue installazioni del valore di 170 milioni di dollari.

La lotta contro l'abbassamento, iniziata da qualche anno con l'immissione di milioni di barili d'acqua (v. pag. 359), era stata negli ultimi mesi un po' rallentata.

A causa della minaccia ai bacini navali, il governo degli USA aveva ripreso, nel mese precedente, il suo progetto, che prevedeva la chiusura di tutti i pozzi di Wilmington. Gli operatori di Wilmington furono accusati di non mostrare molto zelo nell'immettere il flusso d'acqua: invece di 400 mila barili al giorno (pari a 14.560 .000 litri), in Luglio ne erano stati immessi solo 200 mila (7.280.000 litri), come in Gennaio.

Gli operatori locali si sono difesi osservando che dall'Ottobre 1958 erano stati immessi 50 milioni di barili, più della metà di quanti ne erano stati iniettati fino allora.

L'acqua poteva essere iniettata in quasi tutti i 6800 acri (circa 2700 ettari), ma inizialmente gli sforzi erano limitati alla parte superficiale e al fianco Sud.

$\mathrm{Si}$ progettava di aumentare l'immissione a 500.000 barili (18.200.000 litri) giornalieri entro l'anno 1959. Per il Gennaio 1961 era previsto un totale di 238 pozzi d'iniezioni.

L'effetto delle iniezioni d'acqua già si avverte nell'area dove l'immissione è stata effettuata molto a lungo (Fig. 17).

Le tre zone superiori, in alcune parti dei blocchi 3,4 e 5 , ricevono ora circa 180.000 barili di acqua di mare (circa 6 milioni e mezzo di

(*) Da \& The Oil and Gas Journal „ Luglio, Agosto e Dicembre 1959. 
litri), al giorno. Esse sono state a questo livello soltanto per 8 mesi, ma circa 25.000 .000 di barili (910 milioni di litri circa) erano stati iniettati nei precedenti 5 anni.

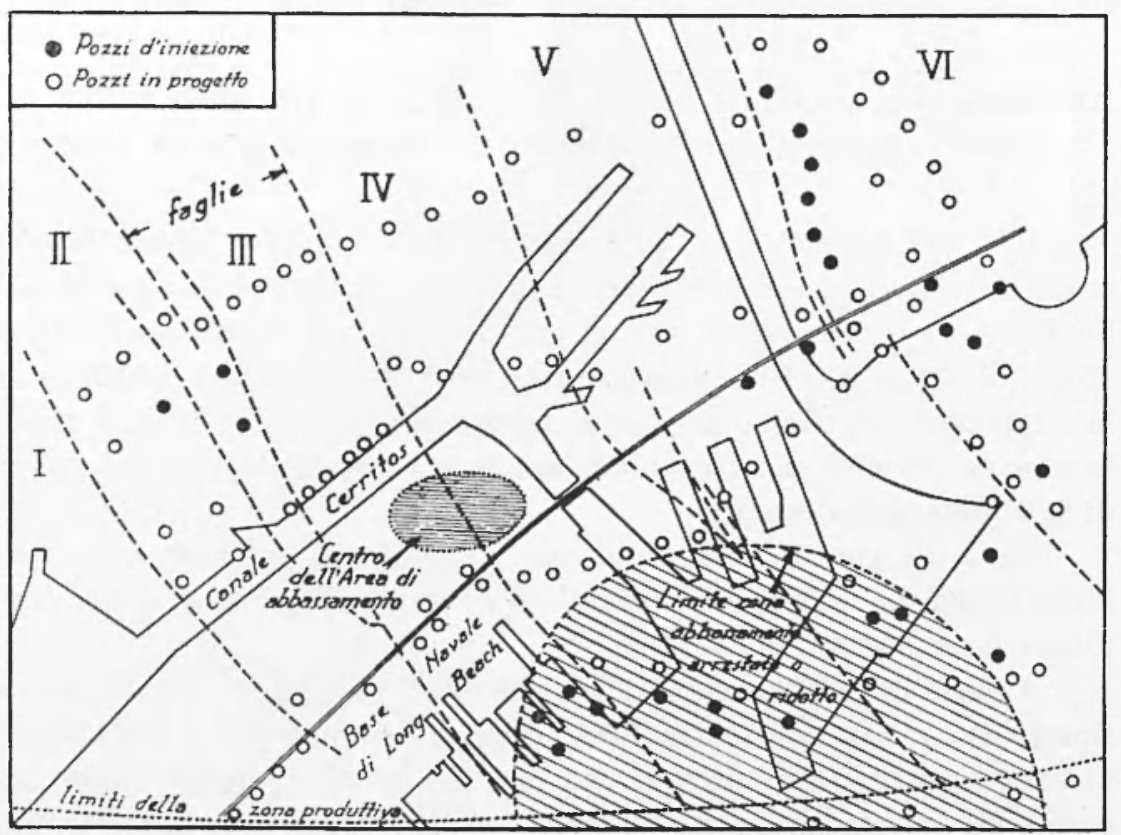

Fig. 28 - Zona di Long Beach, soggetta a forte flessione, determinata dalla grande estrazione di idrocarburi. Le iniezioni di notevoli quantitativi di acqua nel sottosuolo, hanno in parte arrestato il fenonemo di abbassamento. La Figura riporta la posizione dei pozzi d'iniezione funzionanti nel Luglio 1959 e di quelli previsti. (Da a The Oil and Gas Journal, "Luglio 1959).

Una campagna di misure compiuta nell'area del porto durante l'Agosto 1958, ha per la prima volta rivelato che il programma dava i suoi frutti. Il gradiente di sprofondamento nelle vicinanze del molo $A$ era stato ridotto. Una seconda ispezione, compiuta nel Febbraio 1959 provò che l'abbassamento era stato completamente arrestato in un'area di 500 acri (pari a circa 200 ettari) e che il gradiente era ridotto in più di 750 acri (300 ettari) (Figg. 28-29).

Questo successo parziale ha fatto prevedere che lo sprofondamento nell'intera area potrà essere arrestato fra il 1961 e il 1964.

2. - Nell'Agosto 1959 vengono immessi 215.000 barili di acqua di mare al giorno. Per il Luglio 1960 tutto il campo, tranne le estremità 
settentrionale e occidentale, può essere inondato Se tutto andrà secondo le previsioni, a quella data più di 1.000 .000 di barili (più di 36 milioni di litri) di acqua al giorno, saranno immessi nel sottosuolo attraverso pozzi d'iniezione. Si prevede una spesa complessiva di 65 milioni di dollari.

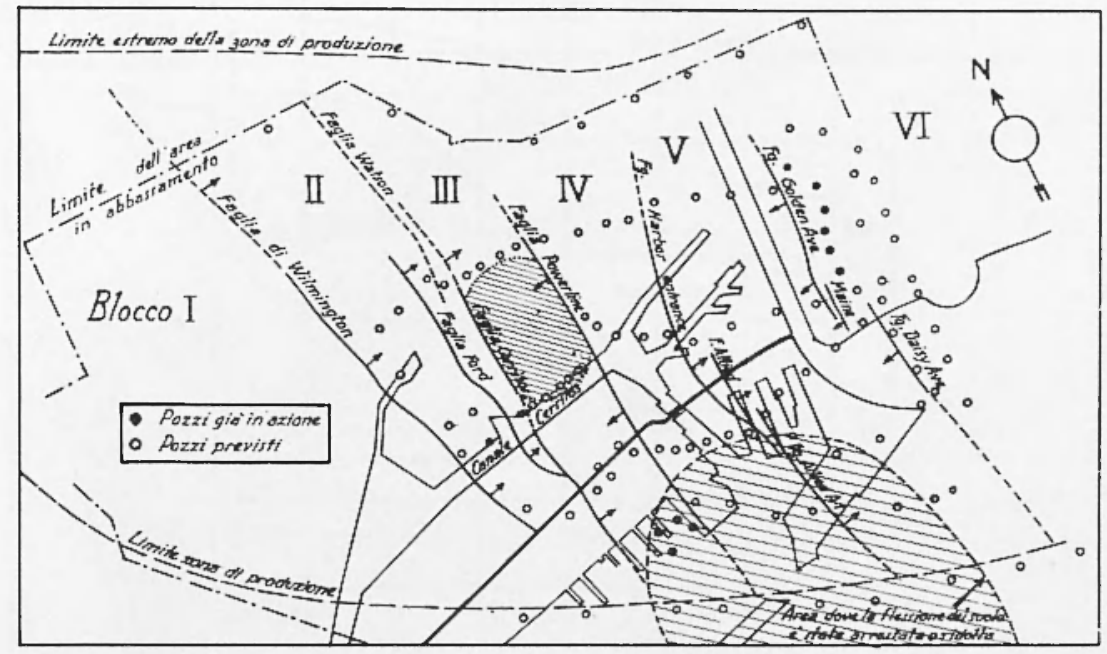

Fig. 29 - Particolari della zona di Long Beach ( dall'estrazione di petrolio e gas dal sottosuolo, causa prima dell'anormale abbassamento ivi verificatosi. (Da "The Oil and Gas Journal", Agosto 1959).

Si spera con ciò di arrestare l'abbassamento del terreno, che ha assunto proporzioni veramente allarmanti. In una parte del campo petrolifero, la superficie del terreno è sprofondata di circa 25 piedi (7,6 $\mathrm{m} \mathrm{ca}$ ) e continua a sprofondare al ritmo di quasi 1 piede all'anno. Se non si interviene, l'abbassamento terminale potrebbe raggiungere 43 piedi (circa $13 \mathrm{~m}$ ) al centro dell'area depressa. Alcuni ingegneri ritengono che $\mathrm{i}$ dintorni di Long Beach possono abbassarsi di circa 5 piedi.

Scopo delle iniezioni di acqua a pressione è quello di restaurare la pressione nel serbatoio (riserva), raggiunto il quale, si renderà necessario mantenere la pressione ottenuta come opportuna misura anti-abbassamento.

Il piano approvato dal supervisore di Stato per il petrolio e il gas, si propone di restaurare, almeno parzialmente, la pressione nella riserva in cinque anni. Ciò richiede l'iniezione, durante questo periodo, di un totale di quasi 2 bilioni di barili (circa 72 miliardi di litri) di acqua 
di mare. $A$ questo scopo si deve arrivare ad un ritmo d'iniezione di approssimativamente 1.113 .000 barili al giorno. Si ritiene, ad ogni modo, che la quantità di 1 milione di barili giornalieri sarà raggiunta nel Luglio 1960.

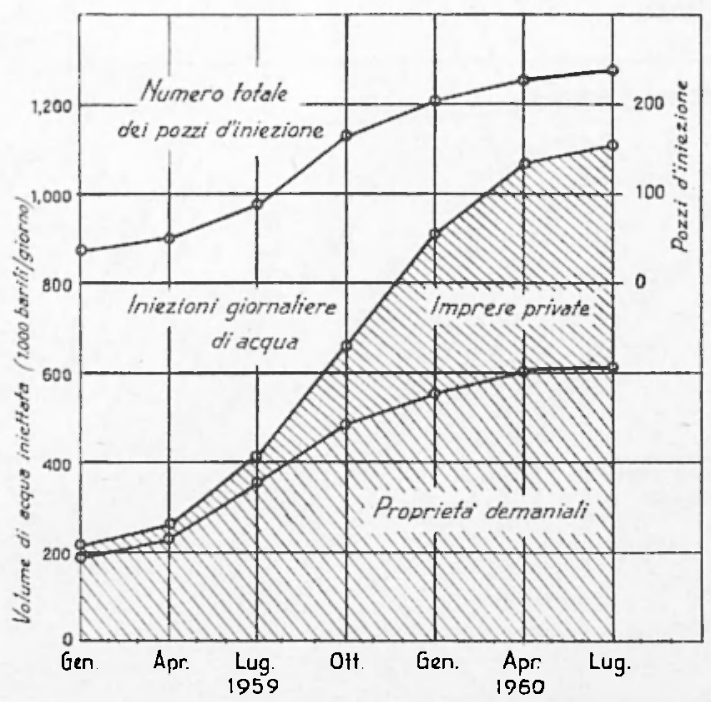

Fig. 30 - Entità d'acqua iniettata durante il 1959 e prevista per il 1960. (Da "The Oil and Gas Journal", Agosto 1959).

Prima del Gennaio 1961 il numero dei pozzi d'iniezione sarà aumentato a 238. Essi saranno distribuiti nei vari blocchi fagliati (Figure 29-31).

È stato constatato che in più punti, dove si è effettuata l'immissione di acqua, è andata arrestandosi la diminuzione della pressione nei pozzi. Contemporaneamente, anche l'abbassamento ha subito sensibili rallentamenti. Un rilievo eseguito nel Febbraio 1959 ha mostrato - come è già stato detto - che l'abbassamento è stato fermato in 500 acri sul fianco meridionale dei blocchi 4 e 5 . In altri 750 acri il ritmo dell'affondamento è stato sensibilmente ridotto.

Quando si presentò la necessità di trivellare molti pozzi nel porto di Long Beach, allo scopo di evitare la parte più congestionata del porto, i pozzi d'iniezione vennero opportunamente inclinati.

3. - Nel Dicembre 1959 due nuovi impianti per iniezione di acqua ad alta pressione sono entrati in funzione nel campo di Wilmington. 
II più potente puó immettere 237.000 barili d'acqua salata al giorno a pressioni di 850,1050 e 1250 atmosfere psi $(*)$, ricevendola alla pressione di 25 psi $(1,5$ atm ca). Il più piccolo ha una capacità di 157.000 barili al giorno. L'aggriunta dei due nuovi impianti porta la capacità totale d'iniezione per l'intero campo a 874.000 barili giornalieri.

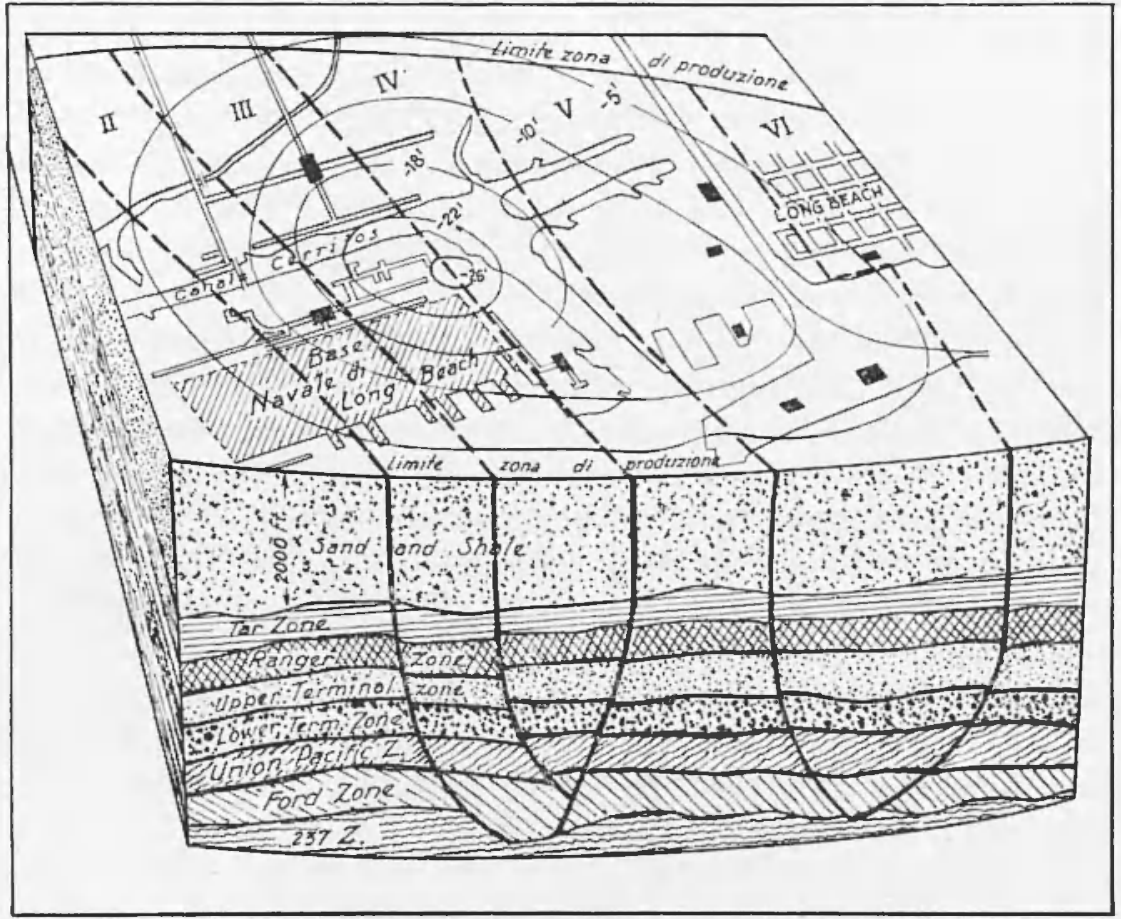

Fig. 31 - Stratificazione del sottosuolo in corrispondenza di Long Beach o delimitazione della zona di più accentuato abbassamento (26 piedi nella parte centrale) (Da "The Oil and Gas Journal", Agosto 1959).

Il completamento di due nuovi impianti porterà ad un totale di più di $1.000 .000 \mathrm{di}$ barili al giorno. Nel Dicembre le iniezioni furono di più di 400.000 barili in media al giorno. Si conta di raggiungere il milione di barili al giorno, nel primo semestre del 1960 (Fig. 30).

(*) psi $=$ per square inch. 
L'estrazione del petrolio e del gas è considerata la causa principale dell'abbassamento. Vari studi hanno mostrato che la quasi totalità della superficie che si abbassa può essere concentrata nelle zone Tar, Ranger e Terminal (Fig. 31). Gli studi hanno inoltre mostrato che l'abbassamento maggriore si è verificato nell'area dove il petrolio e il gas sono stati estratti in maggior quantità.

I due nuovi impianti forniranno acqua per i pozzi iniettori nelle vicinanze del bacino navale di Long Beach, vicino al centro dell'area maggriormente depressa, che ora si estende per oltre 20 miglia quadrate. L'abbassamento al centro dell'area (Fig. 31) ha raggiunto i 26 piedi ( $8 \mathrm{~m}$ circa) e continua. 11 gradiente annuo di abbassamento in questo punto è ora di $9 / 10$ di piede, di contro ai 2,4 piedi del 1951 . Un estremo abbassamento di 45 piedi è stato previsto da alcuni ingegneri, se non verranno intraprese misure correttive.

Che l'immissione di acqua nelle zone costituisca il rimedio auspicato, è provato dai risultati ottenuti presso la banchina $A$ del porto. Periodici rilievi fatti durante gli ultimi due anni mostrano che l'abbassamento è stato arrestato in un'area di 700 acri (280 ettari) e fortemente ridotto in un'area di 1100 acri (440 ettari).

Seguono ampie notizie sugli impianti.

Roma, Gennaio 1960. 


\section{RAFFRONTI E CONCLUSIONI}

1. - A distanza di nove anni dalla mia allarmata (e motivata) relazione del 1959, mi resta poco da aggiungere, a testimonianza della piena validità di quanto in essa andavo affermando.

Prima di trarre le fin troppo evidenti conclusioni, ritengo non inutile fare un raffronto con quanto si è verificato in altre parti del mondo, e particolarmente in California (Appendice I).

A questo proposito, basterà limitarsi a richiamare il parallelismo fra le estrazioni delle acque profonde della California e quelle praticate nel Polesine.

Nella regione del delta padano i pozzi metaniferi si affondano tra i 200 ed i $650 \mathrm{~m}$ e le condizioni dei giacimenti non consentono l'estrazione del gas senza la fuoruscita di acque. Ed è appunto sulla sottrazione delle acque sotterranee, piuttosto che sul gas stesso, che è necessario soffermarsi. Infatti, il gas metano - nella zona - si trova generalmente emulsionato con acqua salmastra in strati sabbiosi. Qualche modesta falda di gas secco, rinvenuta nel passato, specialmente in una fascia più vicina al mare, si è rapidamente esaurita.

Gli strati di sabbia del Quaternario, alternati ad altri di argilla che ne assicurano la copertura, sono originati da sedimenti di laguna e di mare poco profondo. Sono ricoperti in superficie da un certo spessore di deposito continentale, in questo caso alluvionale.

Lo spessore della formazione quaternaria aumenta sensibilmente da Ovest verso Est; infatti dai $500 \mathrm{~m}$ circa nella zona di Rovigo, passa a più di $2.000 \mathrm{~m}$ di spessore alla foce del Po $(3.000 \mathrm{~m}$ ai margini orientali delle valli di Comacchio; $2.400 \mathrm{~m}$ a Codigoro).

E opportuno a questo punto dare qualche ragguaglio sulle modalità di estrazione del metano.

"I giacimenti di metano vengono sfruttati mediante trivellazione di pozzi, a pareti rivestite di colonne tubolari di diametro da 80 a $200 \mathrm{~mm}$, che portano nella estremità inferiore un settore filtrante. I filtri vengono inseriti in modo da coprire uno degli intervalli porosi mineralizzati, esistenti fra i 100 e i $600 \mathrm{~m}$ di profondità. Un orizzonte superficiale con scarse manifestazioni gassose, peraltro noto da moltissimi anni, non viene industrialmente utilizzato.

Orizzonti a profondità oltre i $600 \mathrm{~m}$, benché mineralizzati a gas, normalmente non vengono sfruttati a causa della fine granulometria delle sabbie, che non sono trattenute dai filtri. 
I pozzi vengono messi in produzione insufflando gas metano, mancando ormai, nella quasi totalità dei casi, salienza spontanea. L'iniezione di gas all'interno dei pozzi viene fatta ad una profondità che varia da 80 a $230 \mathrm{~m}$, in modo da creare un alleggerimento della colonna d'acqua sovrastante e stabilire un flusso continuo della emulsione di acqua e gas. Tale emulsione viene scaricata in apposito separatore, montato nei pressi della bocca del pozzo, nella parte alta del quale (duomo) il gas viene raccolto e quindi, con tubazioni, inviato nella centrale di compressione, mentre l'acqua sgorga in basso.

Possiamo quindi constatare come l'acqua venga prelevata dal suolo in grandissima quantità, e come ciò agisca sulle velocità di abbassamento relative alle zone trivellate.

E stato anche reso noto, da studi specifici, come le succitate velocità siano andate aumentando di anno in anno (1951 1962).

L'acqua quindi costituiva, per così dire, il supporto dei terreni polesani, estendendosi, mediante vaste ramificazioni formanti una falda presso a poco uniforme, in tutto il sottosuolo del Delta per una superficie di $50.000 \mathrm{~km}^{2}$ circa. Essa sosteneva praticamente, i suddetti terreni con la sua potente spinta idrostatica.

L'estrazione dal sottosuolo di queste grandi quantità d'acqua che, già intorno al 1957, era per la sola provincia di Rovigo di quasi 230 milioni di $\mathrm{m}^{3}$ - effettuata a mezzo di circa 1.400 pozzi attivi e, nella zona del Delta, di 170 milioni di $\mathrm{m}^{3}$ (900 pozzi attivi) - portava quindi ad un progressivo abbassamento del livello piezometrico (da 20 a $30 \mathrm{~m}$ sotto il piano di campagna) ed inoltre, il rapporto acquagas, soggetto a sensibili variazioni, si aggirava in media da 1,2 a 1,4 " (*).

Come si vede, la tecnica e le profondità di estrazione delle acque metanifere, sono del tutto analoghe alle condizioni di estrazione delle acque irrigue nelle zone della California, soggette a forte abbassamento (Appendice I).

L'analogia delle condizioni di estrazione non poteva tradursi che in una analogia delle conseguenze; e ciò in effetti, si è conformemente verificato.

2. - La Commissione ministeriale (di cui lo scrivente faceva parte), incaricata di indagare sulle cause dell'abbassamento del suolo nel Polesine e nel Delta padano, riconosciuta all'estrazione delle acque meta-

$\left(^{*}\right)$ I periodi tra virgolette sono stati sostanzialmente tratti da una relazione tecnica. 
nifere la causa predominante degli abbassamenti constatati, dopo aspre polemiche e vincendo difficoltà d'ogni genere, è riuscita a fare imporre la chiusura dei pozzi di estrazione, a partire dal Marzo 1960 e con un opportuno programma di sospensione.

Quanto questo, sia pur forzatamente tardivo provvedimento, fosse giusto, fu prontamente provato dai fatti, quali risultano mediamente riassunti nella Fig. 34 ; a mano a mano che l'area di divieto di estrazione delle acque metanifere veniva estendendosi, immediatamente, nelle zone interessate, andava progressivamente diminuendo l'entità degli abbassamenti.

Un altro fenomeno, com'era da attendersi, si è verificato in concomitanza con la sospensione dell'estrazione delle acque metanifere: quello della risalita delle acque nei pozzi. Tale ripresa del livello delle acque segui - cospicua e immediata - la sospensione, dapprima parziale, e quindi totale, dell'emungimento; e prosegui quindi con incremento via ria minore.

Come si è osservato, l'abbassamento del sottosuolo polesano diminuì bruscamente, e sensibilmente, non appena venne a cessare l'estrazione delle acque metanifere (Fig. 34). Naturalmente, non poteva cessare del tutto. Esso prosegui, senza un ordine apparente, restando sensibile solo in prossimità di pozzi ancora in funzione (come provarono i rilievi compiuti fino al 1962,5 ): la sua relocità varia di luogo in luogo e di tempo in tempo.

Non si poteva attendere un arresto brusco e totale dell'abbassamento: troppo abbondante e disordinata era stata l'estrazione delle acque metanifere per oltre un decennio. Quasi certamente, il fenomeno non ha ancora esaurito la sua dinamica.

3. - Quale fu l'entità degli abbassamenti osservati nel Polesine dal 1951 a 1963 ? Le Figure 32 e 33 possono darne una chiara visione. Nella zona che più ha ceduto - da Contarina a Ca' Vendramin, a cavallo del Po di Venezia - l'abbassamento complessivo ha superato i 3 metri e mezzo. Avvallamento enorme, in poco più di un decennio (la quasi totalità essendosi verificata dal 1951 al 1960 , nel periodo cioè di pieno sfruttamento delle acque metanifere). Ma anche in altre località la flessione del terreno fu noterolissima: nella zona di Corbola, p. es., ha superato i 2 metri e cosi nella zona fra Ca' Vendramin e il Po delle Tolle, fra Contarina e il Po della Maestra, ecc.

Gli effetti di tanto anormale abbassamento si sono fatti risentire sull'agricoltura, in conseguenza delle frequenti inondazioni da parte 


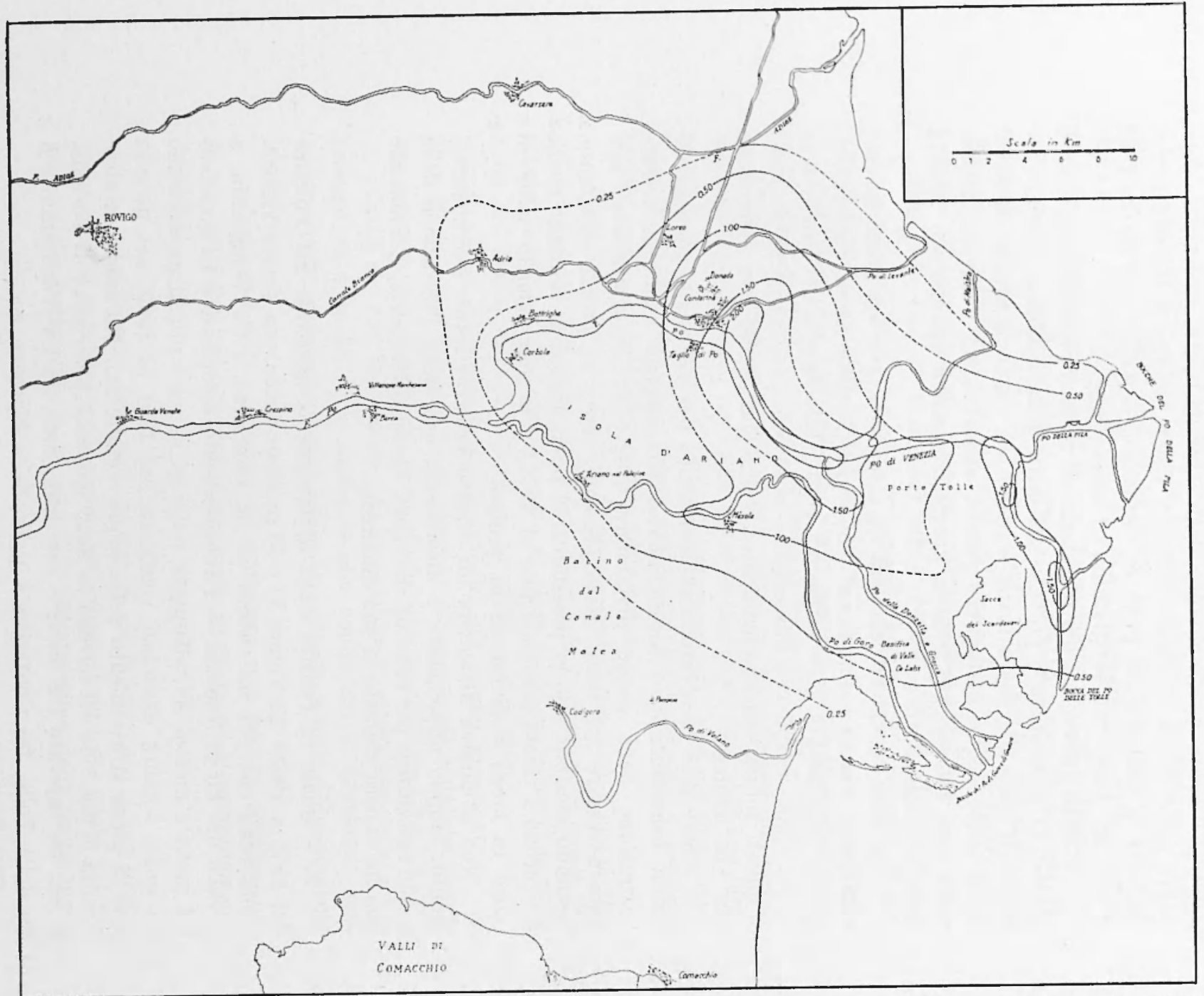

Fig. 32 - Polesine. Delta Padano. Anormali abbassamenti - espressi in metri - verificatisi nel periodo 1950 - 1958. (Dalle elaborazioni del Comitato per le ricerehe sugli abbassamenti del Polesine). 


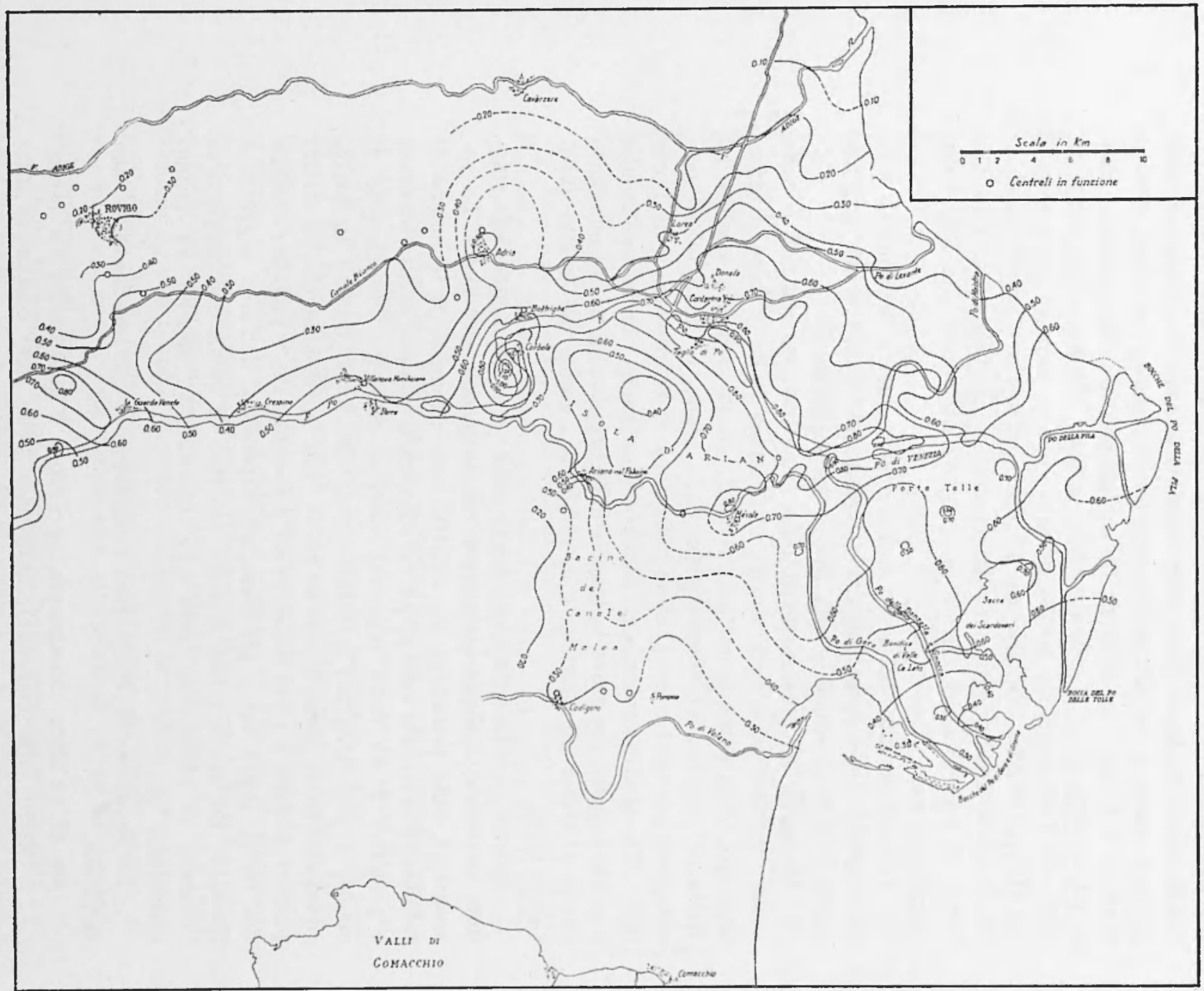

Fig. 33 - Polesine. Delta Padano: Anormali abbassamenti - espressi in metri - verificatisi nel periodo $1958-1963$. Si noti come le isocinetiche abbiano profondamente alterato il loro andamento, nei confronti del precelente periodo, a conferma del migrare lei punti di più accentuata flessione attraverso il Delta, strettamente legata all'emungimento delle acque metanifere.

(Dalle elaborazioni del Comitato per le ricerche sugli abbassamenti nel Polesine). 
del mare e alla conseguente progressiva azione della salinità sui terreni. Ciò ha portato alla sterilità delle terre riscattate alla coltura con imponenti opere e capitali dello Stato e di privati. Del resto, questo pericolo è tuttora incombente nelle zone dove ancora continua l'attività di estrazione: il conseguente, continuo abbassamento mette in difficoltà l'efflusso delle acque, proprio dore lo sgrondo dei terreni è già difficile. Senza contare che l'enorme quantità d'acque salse, riversate disordinatamente nei canali privati e in quelli di bonifica, diffonde la salsedine nei terreni laterali e contribuisce alla loro lenta sterilizzazione.

L'abbassamento del suolo compromette inoltre la stabilità e la funzionalità delle opere pubbliche, indispensabili alle stesse esigenze civili della popolazione e alla sua stessa salvaguardia.

Ma dove le conseguenze si sono dimostrate irreparabili è nella costante minaccia d'inondazione, cui l'intero Delta padano è ormai soggetto. Ogni anno, a partire dal 1951, questa o quella località del Delta, è soggetta ad essere invasa dalle acque, le quali talora permangono per mesi e mesi, come nella zona di Scardovari e di Porto Tolle. Un disagio continuo ed assillante, un'insidia che ha dell'incubo e mette a durissima prova la tenacia delle popolazioni locali, pur tanto ricche di stoica abnegazione e di struggente amore per le loro sventurate terre.

Non ho esitato a definire "irreparabili" le conseguenze delle smodate estrazioni d'acque metanifere del sottosuolo del Delta. La sospensione delle estrazioni ha, infatti, solo attenuato il fenomeno di flessione, e la risalita delle acque nel sottosuolo non può certo restaurare i primitivi livelli della superficie esterna. Soltanto l'immissione di acqua, a pressione, nel sottosuolo potrebbe forse, almeno in parte, riportare i livelli verso le antiche quote. Tale operazione, che ha sortito positivi risultati a Long Beach (Vedi Appendice II), è però impensabile nel Delta, sopra tutto per l'enorme estensione dell'area soggetta a flessione. Ed anche difese a mare, sul tipo di quelle che proteggono l'Olanda, si presentano pressoché inattuabili, almeno su grandi estensioni.

La situazione - è doveroso riconoscerlo - appare seria, se non disperata.

Ma di un'altra conseguenza perniciosa è giuocoforza far cenno.

L'effetto delle estrazioni, indiscusso nelle zone comunque interessate dai pozzi, non è detto non debba estendersi a distanza dai luoghi dove è avvenuta - o tuttora avviene - l'estrazione. 
Non va dimenticato che le flessioni determinate a Long Beach, in conseguenza dell'estrazione del petrolio, si sono sensibilmente estese in zone limitrofe, fino ad interessare la zona di Los Angeles, a circa quaranta chilometri di distanza.

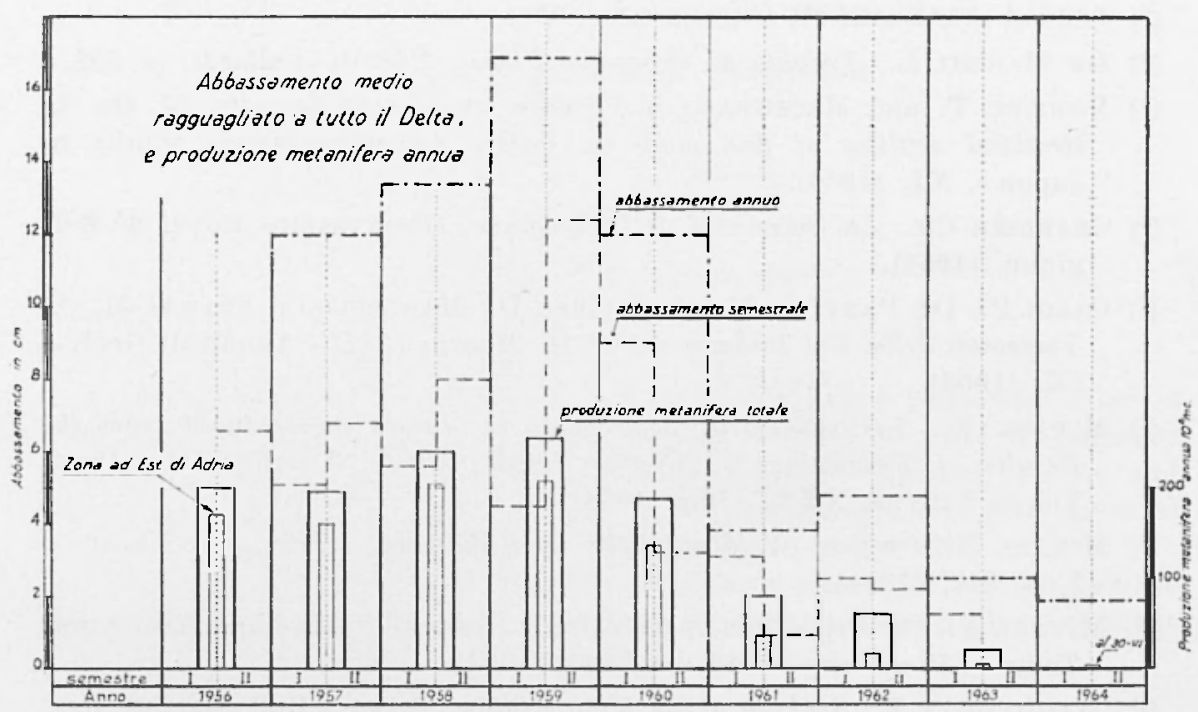

Fig. 34 - Appare netta la correlazione fra la flessione del suolo nel Delta Padano e la contemporanea estrazione delle acque metanifere. (Dalle elaborazioni del Comitato per le ricerche sull'abbassamento del suolo del Polesine e del Delta Padano; per cortese concessione).

Dalle Figg. 32-33 risulta che, a Porto Caleri, a circa $16 \mathrm{~km}$ dalla zona di massima flessione (Ca' Vendramin), l'abbassamento osservato dal 1951 al 1963 è stato di circa $35 \mathrm{~cm}$, avendo raggiunto il valore di ben $20 \mathrm{~cm}$ dal 1958 al 1963, in soli cinque anni, dei quali tre dopo la sospensione degli emungimenti. Si tenga presente che $15 \mathrm{~km}$ più a Nord si trova Chioggia, mentre Venezia è a circa $35 \mathrm{~km}$ da Porto Caleri (e ad una cinquantina dalla zona di massima flessione). A Sud, Ravenna si trova ad analoga distanza da $\mathrm{Ca}^{\prime}$ Vendramin. E noto che, dal 1950 Venezia e, pare, anche Ravenna subiscono, in media, abbassamenti dell'ordine di $1 \mathrm{~cm} /$ anno, mentre abbassamenti di questo ordine si osservavano, prima delle disordinate estrazioni di acque metanifere, nell'intervallo di un secolo.

Molti troveranno queste correlazioni alquanto azzardate. Mi auguro - questa volta, vivamente mi auguro - di essere in torto.

Roma, Maggio 1967. 


\section{BIBLIOGRAFIA}

(1) J und Berlin, p. 72, (1938).

(2) JAHNke E., Eude F., l. c., p. 85, (1938).

( $\left.{ }^{3}\right)$ De Marchi L., Trattato di Geografia Fisica. Editore Vallarli, p. 502.

(3) Nomitsu T. and Matsunaga Y., Studies on marine deposits. II. On the stratified settling of fine mud in water, "Oceanographic Works in Japan ", XI, (1940).

$\left.{ }^{5}\right)$ Charlier Ch., La Séismicité de la Belgique, Observatoire Royal de Belgique, (1951).

(8) Caloi P., De Panfilis M., Di Filippo D., Marcelli L., Spadea M. C., Terremoti della Val Padana del 15-16 Maggio 1951. "Annali di Geof.", IX, (1956).

(i) Mryabe N., Vertical Earth Movements in Japan as Deduced from the Results of Rerunning the Precise Levels. "Bull. Earthq. Res. Inst., Tokyo Univ.", XXX, (June 1952).

$\left.{ }^{8}\right)$ Miyabe N., On Gas - Yelding Wells in Hukagawa, "Proc. Imp. Acad.", 7, p. 347, (1931).

(9) Mixabe N., On Depression of the Earth's Crust in Honzyô and Hulkagawa, Tokyo, "Proc. Imp. Acad.", p. 844, (1932).

(10) Kisinnouxe F., Sinking of the Koto Region, Tokyo. "Bull. Earthq. Res. Institute, Tokyo Univ. ", XXIX, pp. 407, 408, (June 1951).

(11) WaDATI K., Rep. Disast. Res. Inst. Osaka, 2 (1939), 3 (1940).

(12) Wadati K. Hirono T., l. c., 5 (1942).

( $\left.{ }^{13}\right)$ Russel, Phisiography of Lower Mississippi Delta. "Louisiana Geological Survey Bull. ", p. 175, (1936).

(14) DAL PIAZ G. B., Il bacino quaternario polesano-ferrarese e $i$ suoi giacimenti gassiferi. "Atti del Convegno di Milano (30 Settembre-5 Ottobre 1957) su I giacimenti gassiferi dell'Europa Occidentale ", indetto dalla Accademia Nazionale dei Lincei e dall'Ente Nazionale Idrocarburi. Acc. Naz. dei Lincei, 1959.

(15) Gilully J., Grant U. S., Subsidence in the Long Beach Harbor area, California. "Geological Society of America, Bull.", 60, March 1949.

${ }^{\left({ }^{18}\right)}$ Progress Report Land-subsidence investigations San Joaquin Valley, California, through 195\%, a cura dell'" Inter-Agency Committee on Land Subsidence in San Joaquin Valley ", Sacramento, California, pp. 160, 46 Tav. fuori testo (1958).

(17) Poland J. F., Davis G. H., Subsidence of the land surface in the TulareWasco (Delano) and Los Banos-Kettleman City areas, San Joaquin Valley, California, "Ann. Geophys. Union Trans. ", 37, 3, pp. 287 296, (1956). 